This item was submitted to Loughborough's Research Repository by the author.

Items in Figshare are protected by copyright, with all rights reserved, unless otherwise indicated.

\title{
Pubertal development and sedentary behaviour during adolescence.
}

PLEASE CITE THE PUBLISHED VERSION

PUBLISHER

(C) Ian Murdey

LICENCE

CC BY-NC-ND 4.0

\section{REPOSITORY RECORD}

Murdey, lan. 2019. "Pubertal Development and Sedentary Behaviour During Adolescence.”. figshare. https://hdl.handle.net/2134/13542. 


\section{Loughborough University}

This item was submitted to Loughborough University as a PhD thesis by the author and is made available in the Institutional Repository

(https://dspace.lboro.ac.uk/) under the following Creative Commons Licence conditions.

\section{cc) creative}

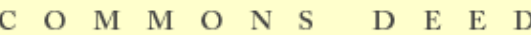

Attribution-NonCommercial-NoDerivs 2.5

You are free:

- to copy, distribute, display, and perform the work

Under the following conditions:

Attribution. You must attribute the work in the manner specified by the author or licensor.

Noncommercial. You may not use this work for commercial purposes.

No Derivative Works. You may not alter, transform, or build upon this work.

- For any reuse or distribution, you must make clear to others the license terms of this work.

- Any of these conditions can be waived if you get permission from the copyright holder.

Your fair use and other rights are in no way affected by the above.

This is a human-readable summary of the Leqal Code (the full license).

Disclaimer 민

For the full text of this licence, please go to: http://creativecommons.org/licenses/by-nc-nd/2.5/ 


\section{University Library}

\section{Loughborough}

University

Author/Filing Title ......uROEEY.......!AN

Class Mark

$T$

Please note that fines are charged on ALL overdue items.

\section{BOR REFERENCE ONLY}

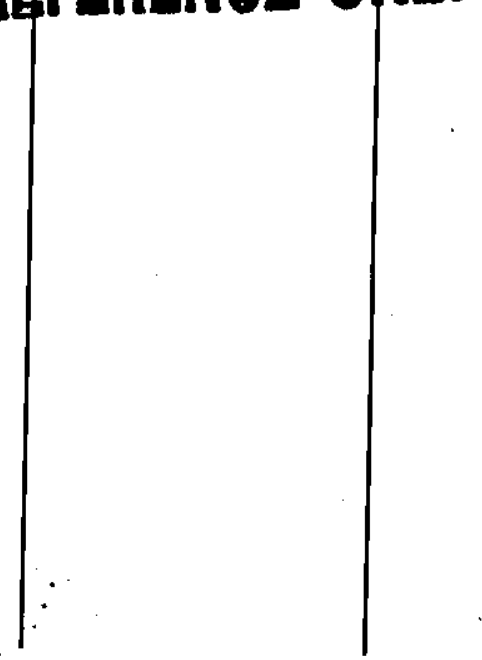




\section{PUBERTAL DEVELOPMENT AND SEDENTARY BEHAVIOUR DURING}

\section{ADOLESCENCE}

by

Ian Murdey

\section{A Doctoral Thesis}

Submitted in partial fulfilment of the requirements

for the award of

Doctor of Philosophy of Loughborough University

30 March 2004

C by Ian Murdey 2004

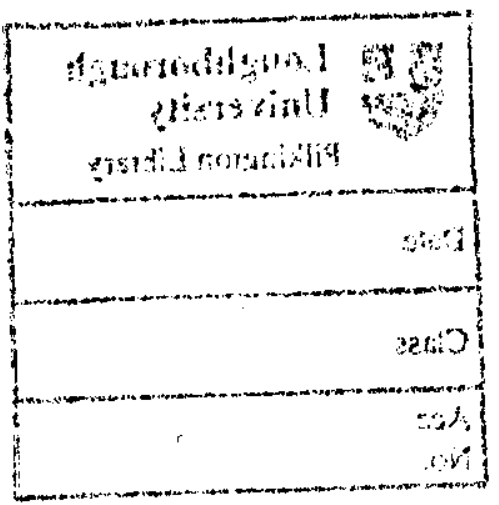




\begin{tabular}{|l|}
\hline $\begin{array}{l}\text { L } \\
\text { Loughborough } \\
\text { University } \\
\text { Pilkington Library }\end{array}$ \\
\hline Date JUNE 2005 \\
\hline Class $T$ \\
\hline \begin{tabular}{l} 
Acc \\
No, 0402941500 \\
\hline
\end{tabular} \\
\hline
\end{tabular}




\section{ABSTRACT}

The objective of the current study was to investigate the relationships between changing pubertal status, body composition, body image, and time spent in sedentary behaviours during adolescence. A mixed-longitudinal design was used involving 119 students from school Years $6(10.0-10.9$ years of age), 8 (12.0 - 12.9 years) and 10 (14.0 - 14.9 years). Participants were assessed in three phases carried out at six-month intervals.

Cross-sectional results from Phase 1 showed that time spent in sedentary behaviours was significantly greater in participants who had started puberty compared with those who were pre-pubertal, and in those who were late-/post-pubertal compared with those who were pre-/early-pubertal. Also, students in Year 10 spent significantly greater time in sedentary behaviours compared with those in Years 6 and 8. These differences disappeared after controlling for the amount of time spent sleeping except for the Year 10 boys. It was concluded that differences in sedentary behaviour during adolescence were not associated with differences in behavioural choice related to greater maturity per se but to a greater opportunity for such behaviours with more waking hours.

Longitudinal analysis examined changes between Phases 1 and 3. Analysis of covariance showed that, for boys only, weekday sedentary behaviour increased by a significantly greater amount for participants who increased puberty compared with those who stayed at the same pubertal level. No differences were found between pubertal groups for either gender during the weekend. No significant correlations were found between changing body composition and changing body image for boys or girls or between the change in pubertal status and change in body composition or body image. Multiple regression analysis showed that a significant amount of the variance of the increase in weekday sedentary behaviour could be explained by increased pubertal status for boys and reduced physical attractiveness for girls. When considering increasing weekend sedentary behaviour an increase in percentage body fat explained a significant amount of the variance for girls. It was concluded that only 
a part of the a priori hypothesis, that changes in sedentary behaviour are associated with pubertal changes, was supported by this data and that any changes found were not associated with behavioural choices triggered by body compositional changes. 


\section{Acknowledgements}

I would like to express my deepest gratitude to my supervisors, Professor Noel Cameron and Professor Stuart Biddle, for all their guidance and advice over the duration of this $\mathrm{PhD}$.

I would also like to extend my gratitude to the Sport and Exercise Psychology group for their continual encouragement and support throughout the three years. In particular Claire Mundy, Sarah Whitehead, Andy Vince, and Simon Marshall for their help with data collection.

Finally, I wish to thank my parents and family who have always supported me in everything I do.

This thesis is dedicated to Jill and Ysa who make it all worthwhile.

"I offer up to you, this tribute." Billy Bragg - Tank Park Salute 


\section{Publications Arising}

\section{Papers}

Murdey, I.D., Cameron N., Biddle S.J.H., and Marshall S.J. (2004). Decreased Sleep provides increased opportunities for sedentary behaviour in adolescents. Journal of Sports Sciences. 22(3), 308-309.

Murdey, I.D., Cameron N., Biddle S.J.H., Marshall S.J., and Gorely T. (2004). Pubertal development and sedentary behaviour during adolescence. Annals of Human Biology. 3I(1), 75-86.

\section{Additional Papers Arising from Project STIL}

Biddle, S.J.H., Gorely, T., Marshall, S.J., Murdey, I., \& Cameron, N. (2004). Physical activity and sedentary behaviours in youth: Issues and controversies. Journal of the Royal Society for the Promotion of Health. 124(1), 29-33.

\section{Conference Communications}

Murdey, I.D., Cameron N., Biddle S.J.H., and Marshall S.J. (2003, September). Decreased Sleep provides increased opportunities for sedentary behaviour in adolescents. Presentation made at the Joint Conference of The British Association of Sport and Exercise Sciences and The British Association of Sport and Exercise Medicine. Sheffield, UK. 


\section{Table of Contents}

ABSTRACT

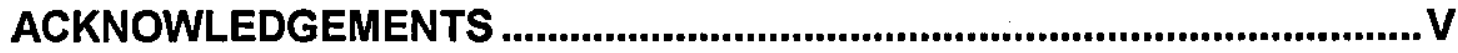

PUBLICATIONS ARISING .................................................................................. VI

INTRODUCTION

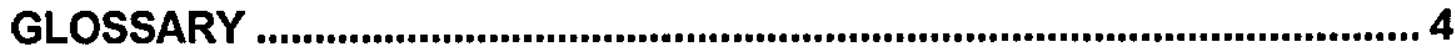

CHAPTER 1: LITERATURE REVIEW .............................................................. 5

Part 1. Sedentarism and Sedentary Behaviours................................................................6

a) An absence of physical activity behaviours ...........................................6

b) A failure to meet activity guidelines....................................................

c) A presence of sedentary behaviours....................................................... 10

d) Physiological characteristics ................................................................ 12

Summary of Sedentarism and Sedentary Behaviours .......................................... 14

Part 2. Inactivity, Adolescence, Growth, and Body Composition ............................15

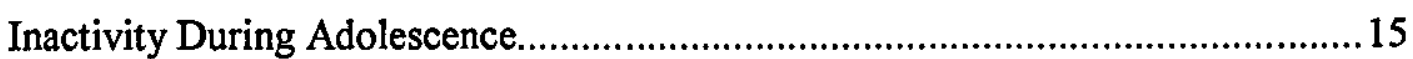

Inactivity, Growth and Body Composition....................................................... 18

Summary of Inactivity, Adolescence, Growth and Body Composition ...................23

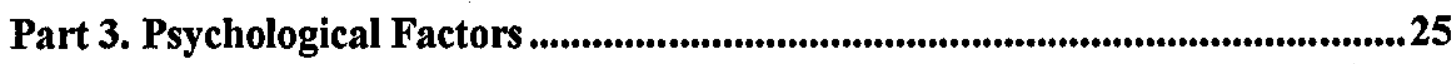

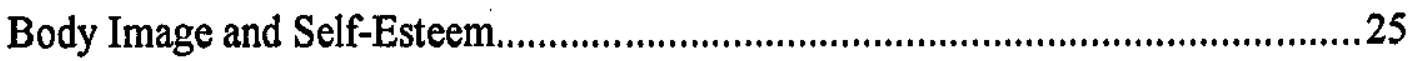

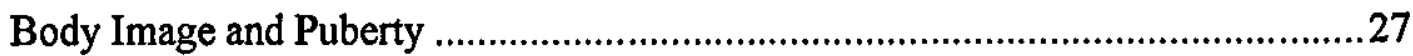

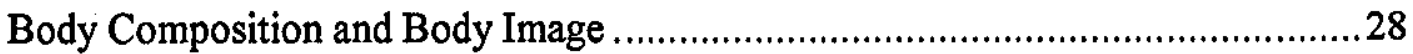

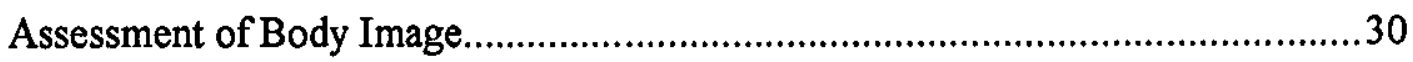

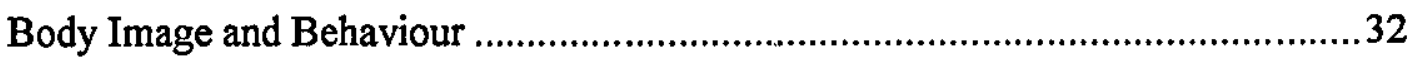

Behavioural Choice Theory ...........................................................................36

Summary of Psychological Factors......................................................................37 


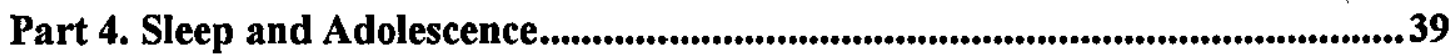

Chapter 1 Conclusions ................................................................................. 40

CHAPTER 2: STUDY DESIGN .................................................................. 41

CHAPTER 3: METHODS REVIEW ............................................................ 44

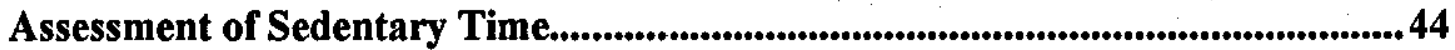

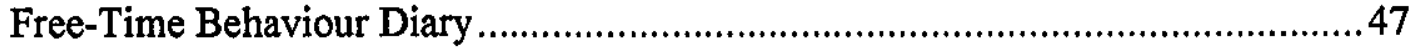

Reliability Testing of the Free-Time Diary ...............................................48

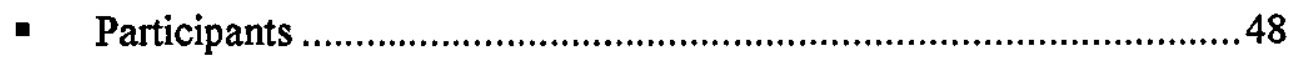

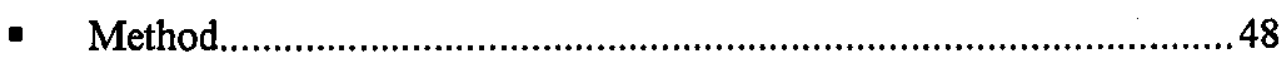

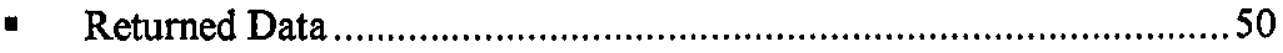

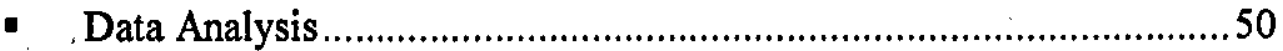

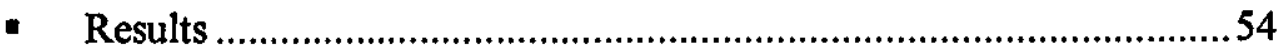

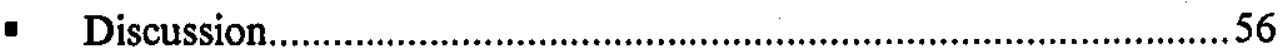

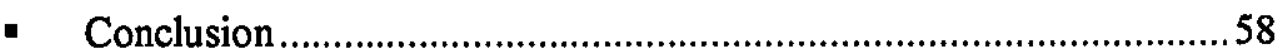

Assessment of Body Composition ...................................................................59

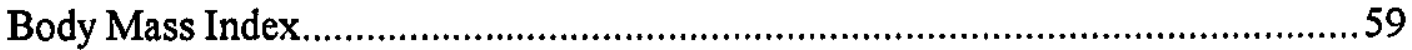

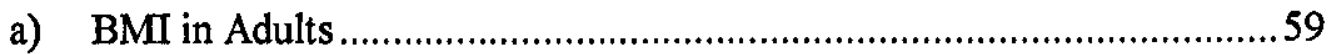

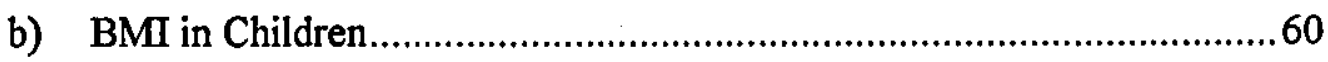

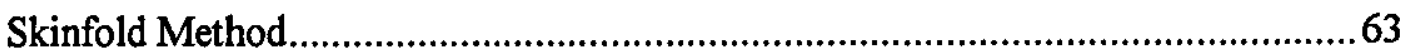

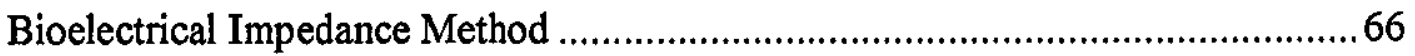

Summary of Body Composition Assessment .................................................71

Assessment of Physical Self-Perception .............................................................. 72

Assessment of Maturation................................................................................. 73

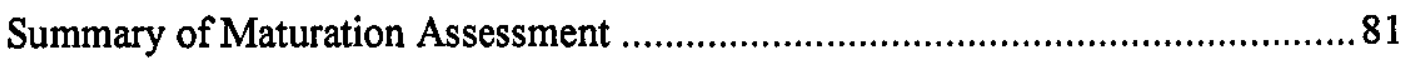

CHAPTER 4: METHODS .................................................................... 83

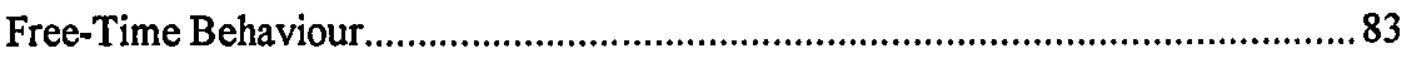

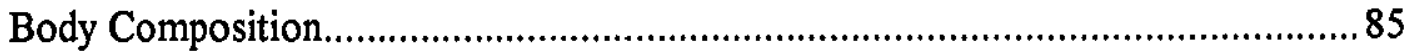

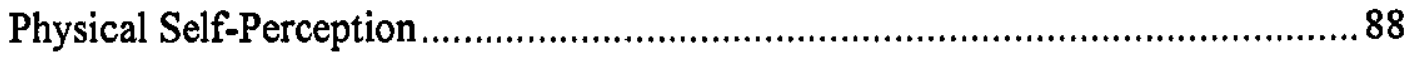


Pubertal Status.

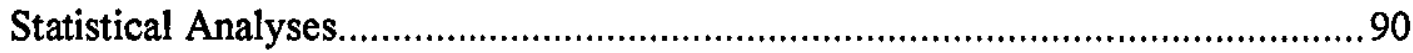

a) Phase 1 (cross-sectional) analyses ......................................................90

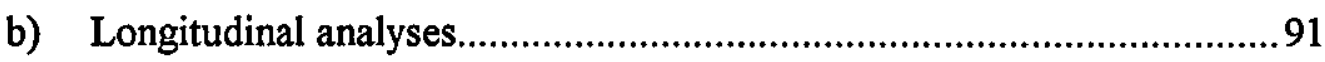

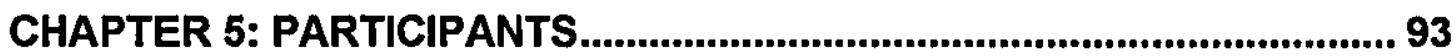

Power Calculation for Estimating Required Sample Size .....................................93

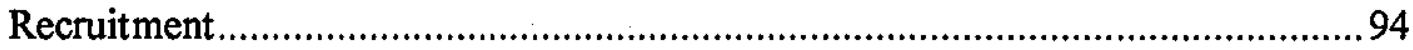

CHAPTER 6: PHASE 1 (CROSS-SECTIONAL) RESULTS .......................... 95

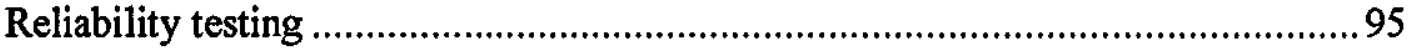

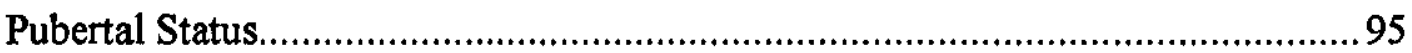

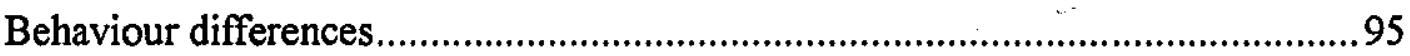

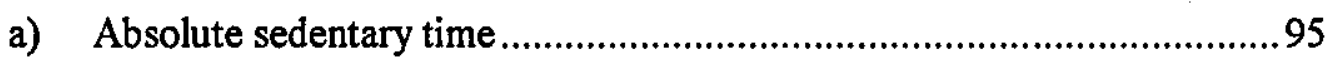

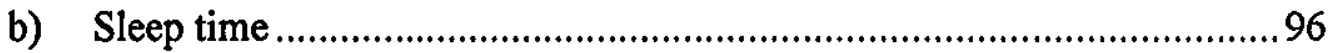

c) Sedentary time after controlling for sleep .............................................96

d) Puberty, body composition, body image, and sedentary behaviour ........96

CHAPTER 7: PHASE 1 DISCUSSION........................................................ 101

CHAPTER 8: LONGITUDINAL RESULTS ............................................... 106

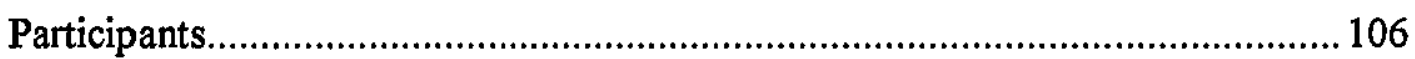

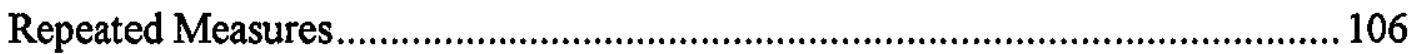

Analysis of Covariance............................................................................ 110

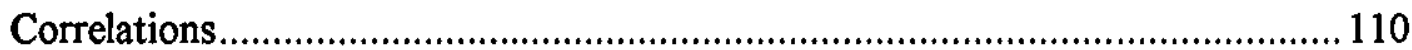

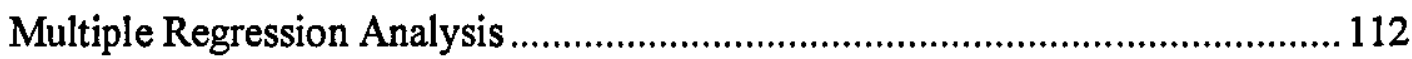

CHAPTER 9: LONGITUDINAL DISCUSSION.......................................... 114

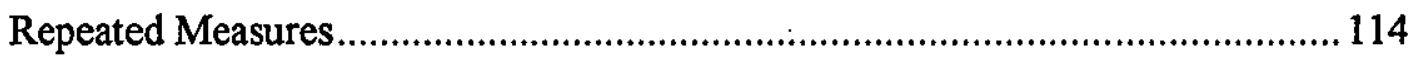

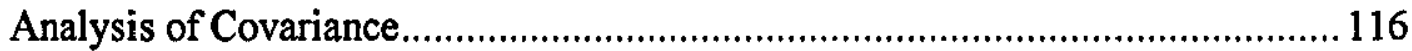

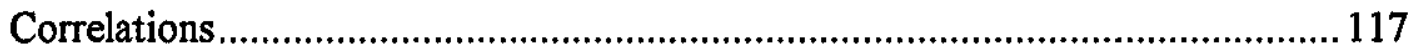

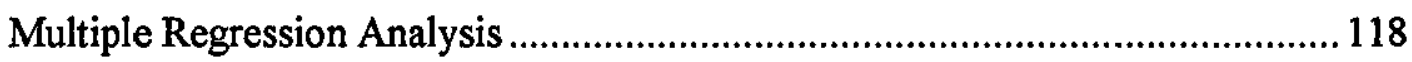

CHAPTER 10: CONCLUSIONS................................................................ 120 


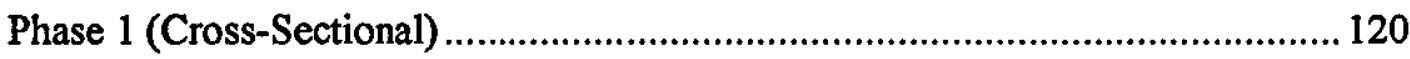

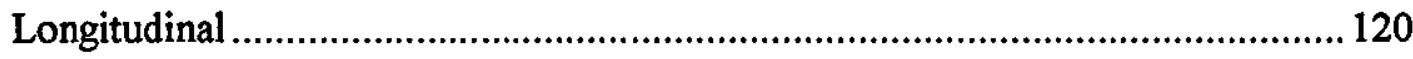

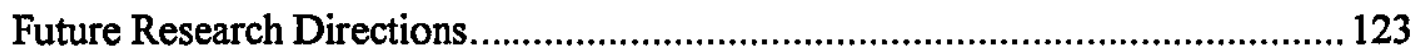

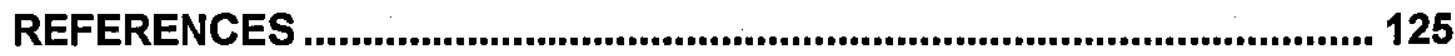

APPENDIX 1: DATA CLEANING..................................................................... 142

APPENDIX 2: ECOLOGICAL QUESTIONNAIRE AND EXAMPLE DAY FROM THE FREE-TIME DIARY............................................................... 144

APPENDIX 3: PHYSICAL SELF-PERCEPTION PROFILE FOR CHILDREN

APPENDIX 4: ANTHROPOMETRY RECORDING FORM .......................... 156 


\section{$\underline{\underline{\text { Introduction }}}$}

Between 1984 and 1998 the prevalence of excess body weight among children in England grew by 150\% (Lobstein, James, \& Cole, 2003). In 1999 approximately 23\% of 10 -year-old boys and $24 \%$ of 10 -year-old girls were classified as overweight, and approximately $10 \%$ and $11 \%$ respectively were classified as obese. For 15 -year-olds these figures rose to $33 \%$ and $29 \%$ overweight, and $16 \%$ and $17 \%$ obese, respectively (Reilly \& Dorosty, 1999). The reduction of adolescent overweight and obesity is an important target because it has been shown that overweight and obese adolescents (body mass index $>85^{\text {th }}$ and $95^{\text {th }}$ age-specific percentiles respectively) have a greater risk of becoming overweight adults than those at, or below, the $75^{\text {th }}$ percentile (Guo \& Chumlea, 1999).

The primary cause of overweight and obesity is positive energy balance - more calories are consumed than are expended (Waters \& Baur, 2003). Physical activity, as a source of energy expenditure, is therefore an important public health issue. Of equal importance is the issue of physical inactivity, or sedentary behaviour, because reducing the time spent in sedentary behaviour will, by definition, lead to an increase in behaviours with a greater energy expenditure. According to Cameron et al. (2003), "While most such programs [to reduce obesity] focus on increasing the time spent engaged in physical activity, it may be more achievable to recommend reducing the time spent in completely sedentary activities such as watching television" (p. 431).

Assessment of time spent in a range of sedentary behaviours, in relation to somatic changes, pubertal status and body image will help gain further insight into the physiological and psychological determinants of such behaviours. This will enable future interventions to be targeted more accurately at the adolescent age group. The current research is a longitudinal study measuring changes in adolescents' free-time behavioural choices over a 12-month period. This is a part of the larger Project STIL (Sedentary Teenagers and Inactive Lifestyles) that aims to assess the nature, prevalence, and determinants of sedentary behaviours in adolescence. 
The a priori hypothesis was that increases in total time spent in sedentary behaviours will be greater in participants who increase their pubertal status, and that body composition and body image will be mediators of this difference via changes in behavioural choices (see Figure 1.1). In other words, as puberty increases, body composition will be changed and this will cause a drop in body image. To minimise any further reductions in body image participants will choose to increase sedentary behaviours. Thus, the relationship between body image and sedentary behaviour is shown as a reciprocal one. Solid arrows in the diagram represent the particular relationships of interest; dashed arrows show other possible relationships. In order to test this hypothesis, the aims of this study were:

- To obtain summaries of total time spent in free-time sedentary activities during a weekday and a weekend day.

- To assess changes in sedentary time within pubertal development.

- To investigate the effects of age, gender, body composition, body image, and sleep on the change in time spent in sedentary behaviours. 
Figure 1.1 The hypothesised relationship between sedentary behaviours and possible determinants.

\section{个Pubertal Status}

个Sedentary

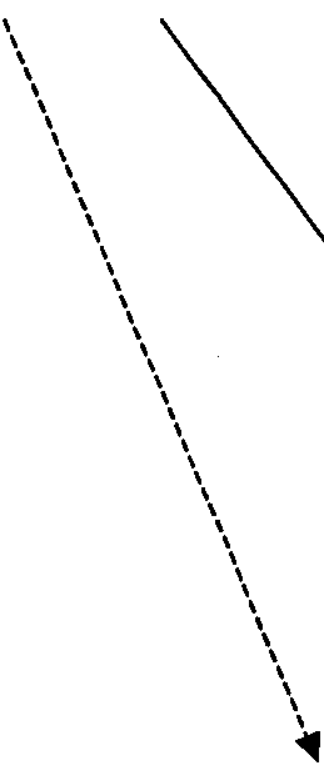

Behaviour

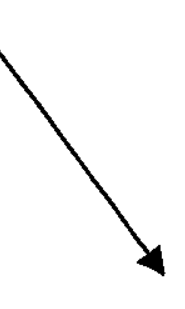

$\triangle$ Body

Composition

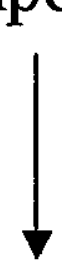

$\downarrow$ Body Image 


\section{Glossary}

Sedentary behaviours - those during which physical activity is minimal (see Table $1.1)$.

Sedentarism/Sedentary - patterns of behaviour that are not active enough to maintain a beneficial level of health. Sedentarism is a characteristic of people who are defined as being sedentary.

Physical inactivity - Dietz (1996) describes physical inactivity as a state "in which bodily movement is minimal" and "for which energy expenditure approximates resting metabolic rate (RMR)" (p.829).

The terms 'physical inactivity', 'inactive', and 'sedentary behaviour' describe behaviour not people (Tudor-Locke \& Myers, 2001); sedentary behaviours form the majority of time spent by most people. Many studies have tried to use a threshold level of behaviour to class participant's as 'sedentary' and these are discussed below. 


\section{Chapter 1: Literature Review}

This review describes current knowledge regarding sedentary behaviours of adolescents. In particular it aims to discuss the definitions of sedentarism and sedentary behaviour that have been used in previous research in order to choose the most appropriate definition for the current study. In addition, this chapter describes the patterns of change of such behaviours throughout adolescence and possible correlates of these changes including growth and psychological factors. The review focuses mainly on the ages 10-16 years as this is the age range studied by the project; age groups that are close to this are also included if their results are considered to be relevant to the current study, other age groups are included where data on adolescents is lacking.

The studies were found by regular computerised searches on MEDLINE (Pubmed), ISI Web of Science, and PsychINFO as well as examination of reference lists in published papers and colleagues' recommendations. The following search terms were used: (childhood OR children OR teenagers OR adolescents OR adolescence OR students OR pupils $O R$ youth OR puberty) AND (inactivity OR sedentary OR television OR video $O R$ computers $O R$ games $O R$ sedentarism $O R$ body image $O R$ self esteem), with the limits (Pubmed only): Title/Abstract, All Child: $0-18 \mathrm{y}$, English, Human. 


\section{Part 1. Sedentarism and Sedentary Behaviours}

This section describes some of the definitions and methods of assessment that previous researchers have used when studying the effects of inactivity.

\section{a) An absence of physical activity behaviours}

A few studies have defined sedentarism as an absence, or a low level, of reported physical activity. Questionnaires are often used to gain information regarding activity habits. These may ask about habitual activity patterns or ask participants to retrospectively describe how much activity they have taken part in over a particular time period. Participants can be classed as sedentary if they fail to report any of the examples of physical activity being studied. Other methods of data collection include interviewer-administered questions, diaries, and proxy reports by parents or teachers.

For example, Kristjansdottir and Vilhjalmsson (2001) asked a random sample of 3600 Icelandic schoolchildren from grades 6,8 and 10 , six questions about participation in gym and swimming classes during school hours, leisure time involvement in physical activity, and time and type of transportation to school. 'All-day sedentary' students were those who did not participate in gym or swimming classes during school hours, participated less than once per week in leisure time physical activity, and were driven to school or cycled/walked for less than 15 minutes one way. Students were 'leisure time sedentary' if they participated less than once per week in leisure time physical activity.

Similarly, Bell (1993) classified boys from a cohort of 12- to 15-year olds as sedentary if they did not participate in competitively organised extracurricular activities and did not consider physical activity to be an integral aspect of their lifestyle. Although Bell reported that regular checks were made by interview and questionnaire to determine the extent and level of physical activity, only activities considered to be 'an integral part' of a participant's lifestyle were used to classify them. This implies that there may have been other, less salient, active behaviours that could have been undertaken by participants classified as sedentary. 


\section{b) A failure to meet activity guidelines}

Other studies have tried to assess the prevalence of sedentary lifestyles by comparing participants' reported levels of physical activity with published guidelines for the amount required to maintain a healthy lifestyle. For example, Pratt, Macera, and Blanton (1999) studied estimates of participation in regular physical activity in adults and young people in the United States. They found that $27.7 \%$ of adults met the recommended levels of activity: that every American should accumulate at least 30 $\mathrm{min} /$ day of moderate intensity physical activity on most days (Pate et al., 1995). Therefore over $70 \%$ of adults in this study were classed as sedentary even though only $29.2 \%$ reported no physical activity at all.

In a study of adolescents' commuting patterns Tuder-Locke, Neff, Ainsworth, Addy, and Popkin (2002) defined participants as sedentary if they failed to meet any of the following guidelines:

Guideline 1: All adolescents should be physically active daily, or nearly every day, as part of play, games, sports, work, transportation, recreation, physical education or planned exercise, in the context of family, school and community activities (Sallis \& Patrick, 1994).

Guideline 2: Adolescents should engage in three or more sessions per week of activities that last 20 minutes or more at a time and that require moderate to vigorous levels of exertion (Sallis \& Patrick, 1994).

Guideline 3: All young people should participate in physical activity of at least moderate intensity for one hour per day (Biddle, Cavill, \& Sallis, 1998).

Care should be taken when using information of this type to quantify levels of sedentarism. In the example of Tudor-Locke et al. (2002) the percentage of students classified as sedentary ranged from $6 \%$ (Guideline 1 ) to $45 \%$ (Guideline 3 ) for the boys and from $7 \%$ (guideline 1) to $51 \%$ (Guideline 3) for the girls. A review undertaken by Sarkin, Nichols, Sallis, and Calfas (2000) found that using different 
questionnaires and interviews led to the prevalence of meeting physical activity guidelines ranging from $32 \%$ to $59 \%$ within the same sample.

It is clear from the reports discussed above (and some discussed below) that studies have used various different definitions of sedentarism making comparison between them difficult. In order to try to solve this problem Bernstein, Morabia, and Sloutskis (1999) developed a definition that they felt could be used universally across studies. They defined sedentary people as those expending less than $10 \%$ of their daily energy expenditure in the performance of moderate- and high-intensity activities. This definition was based on a study involving a population-based sample of 919 residents of Geneva, Switzerland aged 35 to 74 years. In this study the authors defined active persons as those who expend at least $150 \mathrm{kcal}$ per day in moderate-intensity activities. This cut-off point was based on a report from the United States, which suggested that an increase of $150 \mathrm{kcal}$ per day spent in activity of moderate or vigorous intensity is associated with increased health benefits (U.S. Department of Health and Human Services, 1996). With this statement in mind, daily energy expenditure was measured by interviewing each participant regarding all activity carried out the previous day. Specific energy expenditure for each activity was calculated by using the following formula: duration per day (minutes) $\mathrm{x}$ basal metabolic rate multiple $\mathrm{x}$ basal metabolic rate.

Daily energy expenditure was the sum of all activity-specific energy expenditures over the 24-hour period. Moderate/vigorous activity was defined as using 4 or more basal metabolic rate multiples or metabolic equivalents (METs). MET values are calculated as the ratio of the metabolic rate for an activity divided by the resting metabolic rate. The energy expenditure for $1 \mathrm{MET}$ is approximately $3.5 \mathrm{ml} \mathrm{O} / 2 \mathrm{~kg} / \mathrm{min}$ for the average adult.

The results showed that the figure of $150 \mathrm{kcal}$ represented $7.5 \%$ and $6.0 \%$ of females and males average daily energy expenditure respectively. Using the surgeon general's guidelines (U.S. Department of Health and Human Services, 1996), to gain any health benefits a person must expend at least $150 \mathrm{kcal}$ per day in moderate/vigorous exercise, this therefore equates to $7.5 \%$ and $6.0 \%$ of energy expenditure coming from such activity types. In order to correctly identify all sedentary people, Bernstein et al. 
(1999) increased this threshold to define sedentary people as those who expend less than $10 \%$ of their daily energy expenditure in activities using 4 or more METs. The advantage of this definition over those previously discussed is that it can be applied to individuals of all ages, both sexes, and any basal metabolism rates. However, the authors did note that the figures used for moderate activity (4 METs), and the conservative estimate of $10 \%$ for a cut-off point, needed to be confirmed by studies showing the health benefits of using these definitions.

A major disadvantage of this definition is that, like most of the previously discussed articles, it is based on measures of activity rather than directly measuring inactivity. The difficulty with categorising participants as sedentary in this way is that assumptions are being made regarding behaviours that occur outside those that are being asked about. Participants are assumed to be engaged in inactive behaviours at all times when they are not taking part in the specified active behaviours i.e. there is an assumption of an inverse relationship between measured active and inactive behaviours. However, results of previous studies have suggested that sedentary and active behaviours should be studied as separate and distinct constructs. For example, sedentary behaviour in adults (e.g., television viewing) has been found to be independently associated with overweight at all activity levels (Cameron et al., 2003; Salmon, Bauman, Crawford, Timperio, \& Owen, 2000), inactivity tracks better than physical activity from adolescence to early adulthood (Raitakari et al., 1994), and the determinants of adolescent sedentary behaviour have been shown to be distinct from those of physical activity (Gordon-Larsen, McMurray, \& Popkin, 2000; Schmitz et al., 2002). It has also been shown that at the extremes of behaviour (vigorous activity versus inactivity) there may be different mechanisms or associations acting that have independent effects on health (Dietz, 1996).

In addition, Marshall, Biddle, Sallis, McKenzie, and Conway (2002) found that physical activity and sedentary behaviours are able to coexist within the behaviour patterns of adolescents. Using cluster analysis they were able to identify three groups of young people according to their habitual behavioural preferences. Those categories were (1) Techno-Actives (boys) or Sociable-Actives (girls) characterised by high levels of sedentary and active behaviours; (2) Non-Socialising Actives characterised by average levels of sedentary behaviours and high levels of active behaviours; (3) 
Uninvolved Inactives characterised by low levels of reported active and sedentary behaviours. It was concluded that, "Physical activity and sedentary behaviour are not two sides of the same coin" (p. 401).

The finding that some young people have time for both sedentary and active behaviours was repeated in the results of Feldman, Barnett, Shrier, Rossignol, and Abenhaim (2003). They found that, in a cohort of 743 adolescents (mean [SD] age 15.1 [1.2] years), time spent watching television or playing video games was not associated with physical activity. However, physical activity was associated positively with 'productive' sedentary behaviours such as reading, doing homework and working on computers. These results were found to be independent of gender. The authors concluded that some adolescents, particularly those who spend more time in academic pursuits, appear to be able to manage their time better than others.

Within a study such as that from Marshall et al. (2002) it is clear that the definition of 'active behaviours' is more specific than being simply any activity with an energy expenditure above that of the resting metabolic rate. Such conclusions, therefore, do not alter the fact that a reduction in sedentary behaviours must increase energy expenditure via an increase in more active behaviours.

A further difficulty with the use of activity as a proxy for sedentarism is that it is possible that the distinction between physical activity and exercise is not fully understood by participants, who then underreport their physical activity levels. Because these terms are still misused by researchers (Tudor-Locke \& Myers, 2001), then it is certainly conceivable that participants may also misapply them.

\section{c) A presence of sedentary behaviours}

A preferable method of assessing sedentarism, therefore, is to measure sedentary behaviours directly. A further advantage of this method is that it allows assessment of the prevalence of sedentary behaviours and change within-participants rather than simply comparison between groups of active and inactive individuals. This is essential in the current study to investigate the effects of other variables on any such changes. 
The largest body of research has focused on the number of hours spent watching television/video. This profusion of studies may be partly due to the domination that television watching has as a free-time activity, especially for children. A recent report (American Academy of Pediatrics, 1995) stated that in 1993 most children in the United States spent more free-time watching television than any other activity except sleeping. Harrell, Gansky, Bradley, and McMurray (1997) found that, in a study of the most common leisure time activities of 2,200 third and fourth grade children (age range 7 to 12 years, mean age 8.9 years), activities most popular with boys were playing video games (33\%), playing football $(32 \%)$, bicycling $(31 \%)$, watching television (28\%), and playing basketball (26\%). For girls the most popular activities were doing homework (39\%), bicycling (31\%), watching television (30\%), dancing $(27 \%)$, and reading $(23 \%)$. Therefore, in this study although watching television was not the most popular free-time activity for either boys or girls it still rated very highly.

More recently Hofferth and Sandberg (2001) found that, for children aged 6- 12 years of age, television watching was the most popular free-time activity. Whereas for children aged 3-5 years of age television was second only to playing, and for $0-2$ years of age only eating, personal care, day care and playing accounted for more time.

Television is not the only activity that has been used as a proxy measure of sedentary behaviour; Schmitz et al. (2002) assigned a Sedentary Leisure Habits (SLH) score to participants based on their self reported time spent watching television and playing video games, and Waller and Popkin (2002) used measures of hours spent watching television, playing computer games, and using inactive transport to describe the relationship between inactivity and overweight in Chinese children. The importance of video game playing as a sedentary behaviour was highlighted in a study by Phillips, Rolls, Rouse, and Griffiths (1995). They found that in a sample of 429 male and 387 female 11 - 16 year-olds from England, 77.2\% reported playing video games, $24.2 \%$ of players participated seven days per week, and more than $75 \%$ played for longer than half an hour.

However, using these activities alone to measure the prevalence of sedentary behaviour may give rise to under-representation because there are many other sedentary behaviours; for example McKenzie, Marshall, Sallis, and Conway (2000) 
defined sedentary as lying down, sitting, or standing, a definition which may encompass a variety of activities. Alternatively, categorising behaviours as sedentary according to their individual energy costs may be an appropriate method because, in the study of obesity causes, energy output is important. Within 'The Compendium of Physical Activities' compiled by Ainsworth et al. (1993), and later updated (Ainsworth et al., 2000), activities with a MET value of 0.9 - (lying quietly/sleeping) - 1.2 (standing quietly) were classified as inactive. Similarly, Owen, Leslie, Salmon, and Fotheringham (2000) defined sedentary behaviours as those having a MET value between 1.0 and 1.5. Combining the compendium from Ainsworth et al. (2000) and the definition of Owen et al. (2000) gives a list that represents activities involving the minimum of movement and energy expenditure and which are therefore most likely to promote a positive energy balance; some examples from this list can be seen in Table 1.1. Because these MET values have been derived from adult studies caution should be used when applying them to children. It is clear from Table 1.1 that there are many more examples of sedentary behaviour besides watching television/video and playing computer/video games. A purposeful study of sedentarism needs the ability to assess all examples of sedentary behaviours.

\section{d) Physiological characteristics}

Some researchers have used physiological measures, such as heart rate, to assess activity/inactivity levels and describe patterns. A participant is engaged in a sedentary behaviour when their physiological measure drops below a certain threshold. Unfortunately heart rate telemetry has its own limitations, including the facts that;

(a) the gradient of the relationship between heart rate and oxygen uptake depends on a participant's endurance capacity and the type of exercise i.e. whether arms or legs or both are performing the exercise and whether exercise is mainly dynamic or static; and

(b) heart rate can be influenced by factors such as emotional status, body posture, and environmental conditions (Haskell, Yee, Evans, \& Irby, 1993). 
Table 1.1. Examples of sedentary behaviours from the definitions of Ainsworth et al. (2000) and Owen et al. (2000) (adult MET values).

Sleeping (0.9 METS)

Lying quietly, lying in bed (1.0 METS)

Riding in a car/truck/bus, listening to a lecture or music, writing, smoking, watching TV/film (all sitting or reclining) (1.0 METS)

Sitting in a whirlpool (1.0 METS)

Going to the toilet, taking medication, hair or nail care (1.0 METS)

Meditating (1.0 METS)

Standing quietly (1.2 METS)

Reading, talking, bathing, talking on the phone, spectating at a sporting event (all sitting) (1.5 METS)

Reclining with a baby (1.5 METS)

Knitting, sewing, light wrapping, arts and crafts (1.5 METS)

Watering garden (standing or walking) (1.5 METS)

Playing cards/board games (1.5 METS)

Farming (milking by machine) (1.5 METS)

Light office work, typing etc (sitting) (1.5 METS)

Eating (1.5 METS)

Religious activities (1.0 - 1.5 METS)

A useful development could be the integration of a heart rate monitor and accelerometer. Early data from Haskell et al. (1993) demonstrated that the accuracy of estimating oxygen uptake during a wide range of activities can be improved by using a combination of heart rate and body motion data rather than by heart rate alone. Summary variables reflective of different physical activity intensity levels, including sedentary activity, have been published (Janz, Witt, \& Mahoney, 1995), as described in the following extract:

Heart rates of $75 \mathrm{~b} \cdot \mathrm{min}^{-1}$ [beats per minute], $130 \mathrm{~b} \cdot \mathrm{min}^{-1}$, and $150 \mathrm{~b} \cdot \mathrm{min}^{-1}$ were selected to represent sedentary, moderate, and vigorous activity. These heart rates corresponded to accelerometry-derived variables of zero movement counts $\cdot \mathrm{min}^{-1}, 250$ movement counts $\cdot \mathrm{min}^{-1}$, and 500 movement counts $\mathrm{min}^{-1}$ for our subjects. Sedentary activity was set at $<25$ movement counts per minute, 
rather than zero, to minimise the impact of body fidgeting on the overestimation of activity. The daily frequency of physical activity at these intensities was expressed as the percentage of each day spent at sedentary, moderate and vigorous activity. For example, percent daily vigorous activity was calculated by counting the number of minutes spent at movement counts $\geq 500$ and dividing by the sum of the monitored minutes. (pp. 1327-1328)

Sedentary activity was thus set at $<25$ movement counts $\min ^{-1}$, corresponding with $<$ $75 \mathrm{~b} \cdot \mathrm{min}^{-1}$. These results were obtained using a group of 15 boys and 15 girls aged 7 15 years of age and are therefore applicable to children of similar ages although derived from a small sample. The difficulties in using such definitions in large-scale surveys are discussed in Chapter 3.

\section{Summary of Sedentarism and Sedentary Behaviours}

- Reported levels of active behaviours have commonly been used to categorise study participants as active or inactive.

- Such a method is based on the inaccurate assumption of an inverse relationship between measured active and inactive behaviours. It also does not allow assessment of within-participant change in sedentary behaviour.

- A more suitable method of assessing the prevalence of sedentary behaviours during the current study is to measure them directly.

- Sedentary behaviours are best defined as those with an adult MET value of between 1.0 and 1.5 . 


\section{Part 2. Inactivity, Adolescence, Growth, and Body Composition}

\section{Inactivity During Adolescence}

Cross-sectional studies seeking to examine patterns of sedentary behaviour and how they change during the adolescent years have produced equivocal results. For example, Lawrence, Tasker, Daly, Orhiel, and Wozniak (1986) found that, for a cohort of 48 adolescents from Louisiana, average time spent watching television per day was 147 minutes but no significant difference was found between those who were aged $12-14$ years and those who were $15-17$ years. A similar result was reported by Shann (2001) for a racially diverse sample of urban middle school youth aged 10-17 years. Neither hours spent watching television nor hours spent playing video games were affected by the grade of the participant. In addition, Wolf et al. (1993) found that for a multiracial sample of girls of ages $10-17$ years of age there were no school grade-level differences in hours per day of television watching.

In contrast, Myers, Strikmiller, Webber, and Berenson (1996) found that the amount of time spent watching television/videos after school increased significantly $(p<$ 0.001 ) from a median of 120 minutes in Grade 5 (10 years of age) to 180 minutes in Grade 8 (13 years) although total time (before and after school) showed no grade effect. However, Schmitz et al. (2002) found a negative effect of age on time spent watching television and playing video games for girls but not boys, and Heath, Pratt, Warren, and Kann (1994) reported that the percentage of students who watched three or more hours of television per day fell from $45.2 \%$ in Grade 9 (14 years of age) to $30.5 \%$ in Grade 12 (17 years) (significance not reported). Thus the effect of age may vary at different levels of television watching.

One reason for findings that differ between studies may be the differences between the methods of data collection, from family interview (Lawrence et al., 1986), to 24-hour (Myers et al., 1996) or 14-day recall (Heath et al., 1994), to habitual viewing (Schmitz et al., 2002; Shann, 2001; Wolf et al., 1993). However, a consistent weakness of all five studies was the limitation of sedentary behaviours to television/video watching and computer game use only. 
A further weakness of the above studies was the use of cross-sectional data because in order to make any inferences regarding causality it is necessary to use a longitudinal rather than cross-sectional approach. It is also known that information that is needed to inform health policies should come from longitudinal analysis (Armstrong, Welsman, \& Kirby, 2000). Therefore, stronger evidence for any patterns of change in sedentary behaviour during adolescence may come from a review of longitudinal studies.

Bradley, McMurray, Harrell, and Deng (2000) followed 656 participants of mixed gender and race from ages 8-17 years. Annually administered questionnaires were used to assess the three most commonly performed activities. Adult MET values (in the absence of available values for children) of 2,3,5, or $8 \mathrm{METs}$ were used to assign each activity a level of intensity. Sedentarism was defined as reporting at least two of the three activities with METs of 2 or 3 . This was a higher value compared with other definitions (Ainsworth et al., 1993; Owen et al., 2000) that may have led to an overreporting of sedentary behaviour. Pubertal status was measured from 11 years upwards, before this age pubertal status was assumed to be Tanner Stage 1. The researchers found that:

- Sedentary activities were more frequently reported by older participants regardless of gender.

- Less than half of the boys were considered sedentary at any age although it increased from $25.2 \%$ at age $9-12$ to $43.5 \%$ at age $14-17$.

- Females reported more sedentary activities than males overall. From ages $12-$ $15,55.7 \%$ were sedentary and this figure increased each year.

- From ages 9-12 onwards African-American females reported significantly ( $p$ $=.027$ ) more sedentary activities than non-African-American females.

- African-American males began reporting more sedentary activities than nonAfrican-American males from ages $11-14$ years.

- For females aged $11-15$ pubertal status was a significant $(p=.0001)$ predictor of sedentarism, a higher Tanner stage was associated with more reported sedentary activities. 
- TV viewing increased with age for males, becoming the primary sedentary activity by ages $13-16$. In contrast, in females TV viewing was reduced by age and surpassed by more social behaviours.

The major limitations of the study by Bradley et al. (2000) were its assumptions that, a) juveniles and adolescents are able to assess which activities they usually spent the most time doing over the last year, and b) that participants were able to differentiate between activities that were infrequent but of long duration and those that were more frequent but of shorter duration because total time in an activity is a function of both duration and frequency.

Janz, Dawson, and Mahoney (2000) studied a sample of 126 children aged 7 - 11 years who were considered to be pre- or early-pubescent at baseline. These participants were followed for 5 years. Every 3 months, pubertal status, and minutes of television viewing and video game playing during the previous day were assessed during a brief interview. Their results may be summarised as follows:

- Pubertal status had advanced by at least one stage for all participants during the study with over $80 \%$ considered to be late- or post-pubertal by the end. However, direct associations between sedentary behaviour and pubertal status were not reported.

- Females reported a reduction in sedentary behaviour from year 1 to year 5 from $\sim 100 \mathrm{~min} /$ day to $\sim 60 \mathrm{~min} /$ day.

- Males' values fluctuated over the 5 years although there was a downward trend and a small overall decrease from $\sim 100 \mathrm{~min} /$ day to $\sim 80 \mathrm{~min} /$ day.

- Sedentary behaviours tracked from year 1 to year 5 in males $(r=.48)$ but only from year 4 to year 5 in females $(r=.59)$.

In contrast to Bradley et al. (2000), these results suggested that in males sedentary behaviours were more established at an early age and relatively unmodified by puberty, whereas in females sedentary behaviours were reduced. However, the measurement of sedentarism was limited to TV viewing and video game playing and 
therefore may have missed other sedentary behaviours that could have displaced these.

The results of Janz et al. (2000) may be compared to those of Deheeger, Bellisle, and Cachera, (2002) who assessed the time spent watching TV/videos and playing computer games in a cohort of 94 French adolescents followed from 10 to 16 years of age. They found that, in contrast to Janz et al., hours per day spent in these sedentary behaviours increased with age for both genders. In boys the increase was from 1.4 hours at age 10 , to 1.8 at age 14 , and 2.2 at age 16 . In girls the increase was from 1.2 hours at age 10 , to 1.6 at age 14 , and 1.9 at age 16 . Unfortunately no measure of pubertal status was included and, once again, the measure of sedentary behaviour was limited.

\section{Inactivity, Growth and Body Composition}

The relationships between growth, maturity and behaviour are particularly interesting during the period of adolescence because, according to Tanner (1989), "in man only at adolescence do we have anything approaching a general stage [of development]; here, it is true, developments in anatomy, physiology and behaviour tend to occur simultaneously" (p.176). It is the most important period of physical development (Fu \& Hao, 2002), characterised by rapid, and sexually dimorphic, changes in body size, shape, and composition (Rogol, Roemmich, \& Clark, 2002).

Measurement of stature has been the traditional method of growth assessment, however, proxy measurements of body composition (such as skinfold thicknesses) and changes in body proportion can also provide valuable insights into maturation and growth (Rogol, Clarke, \& Roemmich, 2000). Patterns of growth may be compared with population derived growth references such as the body mass index (BMI) charts for UK children produced by Cole, Freeman, and Preece (1995).

A paper that is often cited is the study of Mirwald and Bailey (1986). Fourteen active and 11 inactive Canadian boys were followed from 11 to 15 years of age with a follow up assessment 2 years after the completion of the study. In order to be classified as active or inactive, “...the subject had to have an active or inactive classification in the 
follow-up study, and in four of the five previous assessments" (p. 7). No significant difference was found for height, weight, height velocity or weight velocity. The inactive group tended to be heavier from -5 to +1 years peak height velocity (PHV), although the differences were not significant.

Beunen et al. (1992) studied 32 active and 32 inactive Belgian boys from 13 to 18 years of age. Active boys were defined as, "those who participated in sports activities outside of compulsory physical education for more than $5 \mathrm{~h} . \mathrm{wk}^{-1}$ during each of the first 3 years of the study" (p. 577). In contrast, inactive boys were defined as, "those who participated in no more than 1.5 h.w $k^{-1}$ during each of the first $3 \mathrm{yr}$ of the study outside of compulsory physical education" (p. 577). Assessment of activity level was based on a written questionnaire and interview. There was no difference between groups for socio-cultural characteristics: degree of urbanisation of residence, educational and professional level of the father, family size and birth order. Any differences in chronological age at each measurement were not significant. Anthropometric measurements included body weight, stature, sitting height, skeletal breadths, girths, and skinfolds. The results showed no significant differences between the groups for growth in somatic dimensions, including skinfolds, age at PHV, or skeletal maturation. Like some other papers already discussed, this study included a measure of behaviour which may have failed to pick up less structured activities that could have affected results. A more detailed measure of inactivity may therefore have been advantageous.

A short-term longitudinal study was carried out by Bell (1993) in order to compare a variety of auxological and physiological characteristics of active and sedentary boys between the ages of 12 and 15 years. Participants were considered to be active if they participated in competitively organised extracurricular school games and considered physical activity to be a natural component of their lifestyle. Conversely, participants were classified as sedentary if they did not participate in competitively organised extracurricular activities and did not consider physical activity to be an integral aspect of their lifestyle. The limitations of this classification system have already been discussed (see p.6). There were 33 participants in the active group and 15 in the control (inactive) group although there was a small degree of attrition and addition over the 3-year period. 
Annual measurements were taken of stature and body mass. Peak height velocity was assessed by dividing the period of observation into subintervals and fitting separate, overlapping third-degree polynomials at 3-monthly intervals; the largest observed velocity and the age at which it occurred was used as the estimate of PHV. Somatotype was classified according to the method of Carter and Heath (1990). There are three methods of obtaining a Carter-Heath somatotype:

1. The photoscopic somatotype. Visual inspection of a somatotype photograph, in addition to information of the participant's height and weight, and with reference to a table of somatotypes according to height/weight ${ }^{3}$.

2. The anthropometric somatotype. Calculated from 10 anthropometric dimensions (height, weight, 4 skinfolds, 2 girths and 2 breadths).

3. The anthropometric plus photoscopic somatotype.

Although Bell (1993) did not give details of which method was used, the measurements that were taken suggest that it was method 2.

The results showed no significant difference in height before, during and after PHV between the two groups. No significant differences in age were found for PHV. However, significant differences were seen for level of PHV, 8.5 vs. $9.5 \mathrm{~cm} /$ year for inactive vs. active $(p<.05)$. Velocity of growth was also significantly higher in the active group after PHV $(\mathrm{p}<.05)$.

The active group was lighter than the inactive group before PHV (not significant). However, at PHV and beyond the active group became heavier and the difference became significant at +1 year PHV. The change (increment) in body mass was only significantly different between the groups in the year directly preceding $\mathrm{PHV}(\mathrm{p}<$ .01). The peak body mass velocity (PBMV) was not significantly different between the two groups, nor was the age at PBMV. Descriptive statistics for the somatotype components may be summarised for the two groups as follows:

In the inactive group: 
- Endomorphy showed an overall increase during adolescence but with decreases either side of PHV.

- Mesomorphy rose initially between -2 and -1 years PHV but fell thereafter.

- Ectomorphy decreased between -2 and -1 years PHV, then rose until +1 year PHV before falling to its lowest level.

In the active group:

- After a slight rise between -2 and -1 years PHV, endomorphy fell during subsequent growth.

- Mesomorphy and ectomorphy stayed fairly constant throughout adolescence, although at the time of PHV mesomorphy displayed a slight peak whilst ectomorphy displayed a slight trough.

The only significant differences were:

- Mesomorphy was greater in the active group only at PHV $(p<.05)$.

- Ectomorphy was greater in the inactive group at PHV $(p<.01)$, and +1 year PHV $(p<.05)$.

- Change in ectomorphy was greater for the inactive group in the year preceding PHV $(\mathrm{p}<.05)$.

- Considering somatotype as a gestalt, significant differences were found at PHV between active and inactive $(2.4-4.1-3.9$ vs. $2.1-3.5-4.9)$.

Overall, the inactive group were prominently mesomorphic ectomorphs (73\%). This type accounted for $40 \%$ (non-significant difference between groups) of the active group whilst $31 \%$ were ectomorphic mesomorphs. Bell (1993) suggested that this second category (dominant mesomorph), which was poorly represented in the inactive group, may be more suited to the rigours of contact games. The active group had the more stable somatotype during adolescence.

The results of Bell (1993) can be compared to those of Beunen et al. (1992), and Mirwald \& Bailey (1986) in that there were few differences in growth and maturation between active and inactive participants during adolescence. No study presented specific data regarding those who may have changed category during the 
measurement period. It would have been interesting to assess whether any particular body types were more likely to increase or decrease inactivity during adolescence because this may have implications for future interventions.

As a part of a larger study, Robinson et al. (1993) collected data for 279 adolescent girls (mean age 12.37 years at baseline, S.D. 0.73). Measurements for television viewing, physical activity, sexual maturity, BMI, and triceps skinfold thickness were taken at baseline and follow up at 7, 14, and 24 months. Television viewing was measured by self-report questionnaire in addition to 12 other activities. Participants were required to indicate, on average, how many hours per day they watched television by circling an answer from $0-5$ hours in half hour increments. Level of physical activity was measured by three questions regarding frequency of exerciseinduced sweating and self-assessment of activity level compared to peers.

The results showed that the baseline level of hours of after school television viewing was not significantly associated with baseline or longitudinal change in BMI (adjusted by pubertal stage), triceps skinfold thickness, or level of physical activity over time. In other words, during adolescence level of sedentarism (as measured by television viewing only) was not associated with body composition in this group of girls. However, the limitation of using a single behaviour as a proxy measure of sedentarism has already been discussed in Part 1 .

In contrast, within a cohort of 6149 girls and 4620 boys, Berkey et al. (2000) found a 1 -year increase in BMI was larger in those who reported more time with television, videos, and computer games. Also, Berkey, Rockett, Gillman, and Colditz (2003) found that, for 11,887 boys and girls aged 10 to 15 years, an increase in inactivity was associated with increasing BMI (adjusted to take into account maturational factors) in girls but not boys. The authors stated that although longitudinal studies are not able to provide conclusive evidence of causation they "would provide stronger evidence for causes of weight gain or loss than studies that do not analyze within-child changes in activity and inactivity" (p.836).

Furthermore, Tremblay and Willms (2003) added that caution must be used when "speculating on causation. It may be that children who are prone to overweight and 
obesity may also be prone to living a more sedentary lifestyle; that is, obesity and overweight can discourage children from participating" (p. 1104).

\section{Summary of Inactivity, Adolescence, Growth and Body Composition}

The results of studies that measured inactive behaviour during adolescence show the following general patterns:

- Most associations being made with increases in age rather than maturity.

- Equivocal patterns of inactivity with age i.e. increases in sedentary behaviour coupled with inconsistent changes in TV/video watching and computer use.

- TV/video watching and computer use greater in males compared with females.

- More inactivity among African-Americans compared with their non-AfricanAmerican counterparts.

The equivocal patterns of inactivity change with age add strength to the argument that, in order to obtain more accurate assessments of the prevalence of behaviours likely to affect health, future longitudinal studies should include tools that are capable of measuring a wider variety of sedentary behaviours than simply TV/video watching and computer use. Appropriate measures of pubertal status should also be included.

The majority of studies discussed above implied that there are few differences in growth between active and inactive participants during adolescence. Results for body composition were fairly equivocal however, particularly the BMI-sedentary behaviour relationship although the results may be misleading due to poor measures of activity and inactivity. In particular, Berkey et al. (2000) restricted measurement of inactive behaviours to self-assessment of the typical amount of such behaviours within the year between the two BMI measures. Of course, the advantage of such a method of recall is the large numbers of participants that can be involved compared with an approach involving a more detailed measurement, and the large cohort numbers were a particular strength of this study. 
An additional weakness of some of the studies discussed is that placing participants into groups according to activity level will only identify behaviour changes if a participant moves groups, and activity changes within a group are less likely to be seen. Rather than dividing samples into active and sedentary groups future studies should attempt to assess changes in all participants' activity levels during adolescence. This approach will allow correlates of behaviour and behaviour change in this age group to be more clearly identified including any associations with certain somatotypes.

Using such methods, Berkey et al. (2003) found increases in inactivity to be related to increases in BMI. It should be noted, however, that the results of this study were based on recall of television/video watching and computer game playing only and a closer analysis indicates the possible folly of restricting analysis to too few behaviours. For example, had only video watching been used to model inactivity then a positive relationship would have been found with increasing BMI in boys; a relationship that did not exist when the other behaviours were added. Inclusion of yet more behaviours may have led to a different conclusion again. 


\section{Part 3. Psychological Factors}

\section{Body Image and Self-Esteem}

In addition to physical changes, psychological factors may be important determinants of sedentary behaviour. Adolescence is a time when individuals strive to create an identity for themselves that conforms to the requirements of self, peer, and cultural standards, and the physical self provides the projection of this identity into the public domain (Page \& Fox, 1997).

Figure 1.2. Hierarchical Model of Self-Esteem Structure (Fox \& Corbin, 1989)

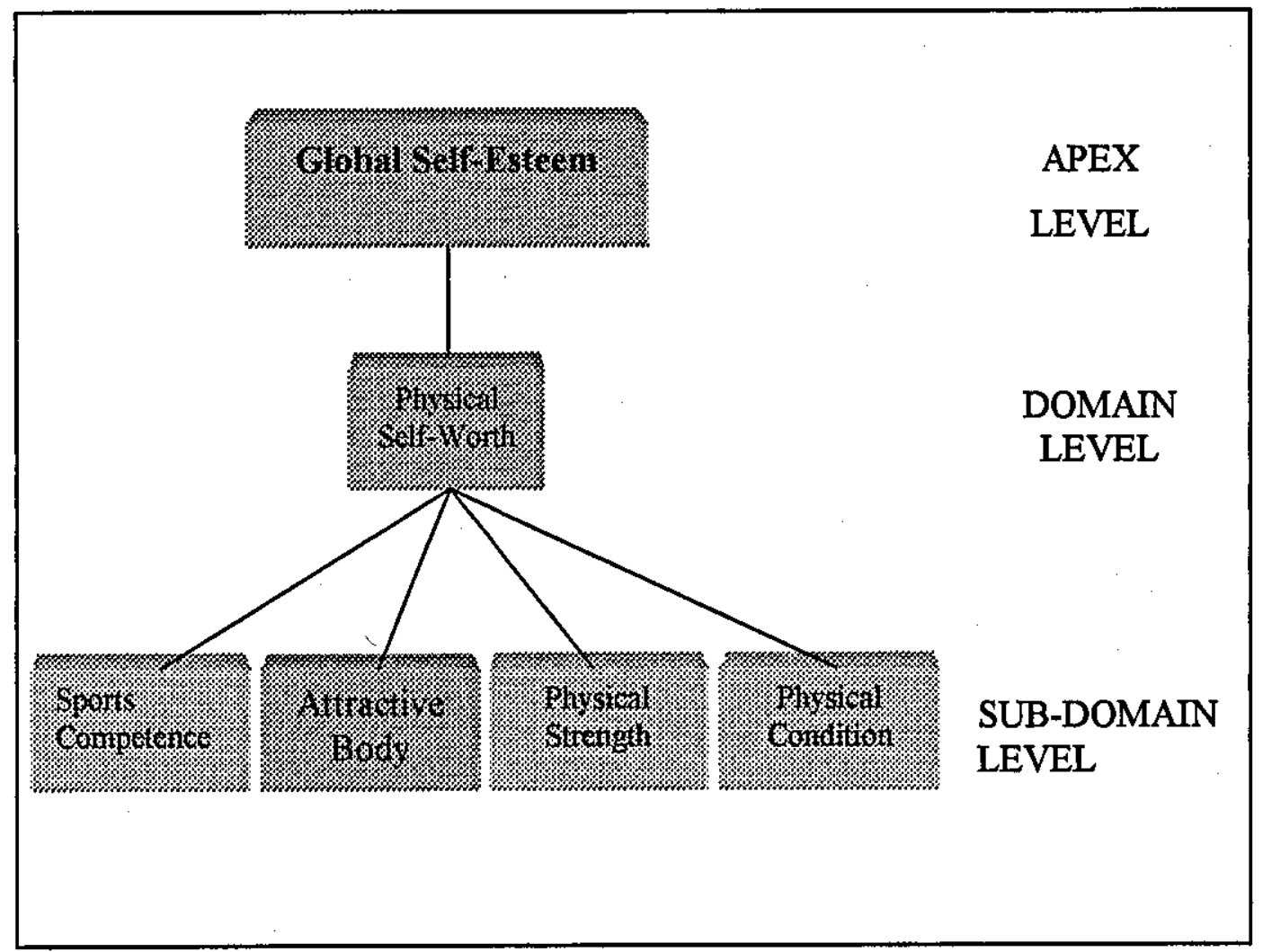

Satisfaction with the whole self is measured with the concept of self-esteem and the development of the physical self is known to be an entity that plays an integral part in the structure of the whole. For example, one particular hierarchical model shows 'Global Self-Esteem' at its apex with 'Physical Self-Worth' below this and underpinned by four physical sub-domains, such as self-perceptions of body 
attractiveness and sports competence (Figure 1.2). These sub-domains were identified by Fox and Corbin (1989) using an open-ended questionnaire with data reduced by content analysis. Multiple regressions showed that the sub-domains explained $68 \%$ of the variance in Physical Self-Worth for males and $69 \%$ for females.

Analyses have shown correlations in children between physical self-worth and global self-esteem to be $r=.60$ for males and $r=.62$ for females (Whitehead, 1995). During free-time it is common to choose behaviours that increase or maintain positive selfesteem (Sonstroem, 1997). This may be achieved by behaving in such a manner that maximises positive, and minimises negative, feelings of physical self-worth.

The assessment of physical self-worth has been dominated by the construct of selfperceptions of body attractiveness or body image (Fox, 1997). Whitehead (1995) found correlations between physical self-worth and body image to be $r=.72$ for males and $r=.77$ for females. This implies that there is also a relationship between body image and global self-esteem, an implication that is supported by the findings of Harter (1993), who found correlations between appearance and global self-esteem as typically between $r=.70$ and .80 . Because global self-esteem can influence behaviour, it may therefore be possible that there is a link between body image and sedentary behaviour.

Two important concepts when considering the impact of body image on self-esteem are centrality and discounting. The idea of centrality suggests that associations will only be found for adolescents who consider their appearance to be of importance and that it may be that some simply do not worry about their bodies in comparison to other aspects of their lives (Page \& Fox, 1997). Discounting is a self-enhancement strategy by which individuals attach a low importance weight to those domains where low competence is perceived. This prevents shortfalls in competence from impacting on self-esteem. However, Harter (1993) states that, in studies involving participants of a range of ages, her colleagues and herself have repeatedly discovered that "selfevaluations in the domain of physical appearance are inextricably linked to global self-esteem. The correlations between perceived appearance and self-esteem are staggeringly high and robust across the life span, typically between 0.7 and 0.8 " ( $p$. 95) and "the evaluation of one's looks takes precedence over every other domain as 
the number one predictor of self-esteem" (p. 96). The lack of discounting of physical appearance even when it scores low on ratings maybe because it is considered so culturally dominant and important (Fox, 1997).

This relationship between body image and self-esteem may be complicated by other factors such as gender. For example, results from Wadden, Brown, Foster, and Linowitz (1991) showed that adolescent females worried most about looks, figure, weight, and popularity and relationships with the opposite sex whereas adolescent boys worried most about money, looks, and popularity and relationships with the opposite sex. In addition, females scored higher than males on degree of worry on 9 out of 15 items including weight, figure and looks. Weight ranked $13^{\text {th }}$ out of 15 for males and physique was only $6^{\text {th }}$, below 'the future'. Despite these gender differences, looks can be seen to rank highly for both sexes. It is interesting to note also that overweight females and males both tended to worry more about weight and figure/physique than their non-overweight peers, and overweight boys also reported greater worry about their health.

\section{Body Image and Puberty}

Gender also has a profound effect on changes to body image during puberty. Data from Harter (1993) showed that for females, perceptions of physical attractiveness fell as age increased from 8 to 16 years of age with no concurrent fall for males. Additionally, McCabe, Ricciardelli, and Finemore (2002) found that for Grade 7 and 9 students (mean ages 13.22 years for males and 13.21 years for females), females displayed significantly less body satisfaction than males and that for the Grade 9 females body satisfaction was less, and pubertal development greater, than for those in Grade 7. For the boys, the importance placed on body image was less in Grade 9 compared with Grade 7. The results of Cohn et al. (1987) with 288 females aged 10.5 to 15.0 years, showed that girls at more advanced pubertal levels displayed a greater discrepancy between current and ideal figures than did less developed girls. However, contrasting evidence was presented by Brooks-Gunn and Warren (1988), who found that in eighty-two 9- to 11-year-old girls in the early stages of puberty, breast development was significantly and positively associated with superior adjustment, peer relations and body image although the same associations were not found with 
pubic hair growth. In a study with 434 adolescent boys aged between 11 and 16 years, Ricciardelli and McCabe (2003) found that puberty and BMI were not significant predictors of any body change programmes including weight loss, weight gain, and muscle gain strategies.

According to Page and Fox (1997), the drop in body image seen for females may be because those parts of the body that are subject to the greatest change are also those which form the basis of judgments of overall attractiveness, for example, the hips, thighs and buttocks, which also tend to be areas of increased fat storage. To quote Hermes and Keel (2003), "The increase in adiposity that accompanies puberty causes young girls' bodies to look less like the cultural thin ideal of beauty" (p. 465). They found that increased maturity in girls was associated with higher BMI and body dissatisfaction. Changes in body composition and regional distribution of body fat during puberty are largely sexually dimorphic however, and the same effect may not be seen in males for whom the percentage of body fat falls during adolescence as the fat free mass increases (Rogol et al., 2002).

Timing of maturation may be an additional important factor in the development of body image. As boys mature their perceived ideal male figure increases in size (Cohn et al., 1987). Early maturing males may therefore increase their body image (Lintunen, Rahkila, Silvennoinen, \& Österback, 1988) as they develop a body that is more muscular (Rogol et al., 2002). This is not true for early maturing females, who are more likely to be taller, wider and fatter than their peers, and for whom perceived body size does not develop together with preferred body size resulting in increased body dissatisfaction (Cohn et al., 1987). For late developers opposite patterns may be present. Page and Fox (1997) speculated that late maturing males may feel higher levels of body dissatisfaction as they desire greater muscle bulk whereas for late developing females the pre-pubertal body type may match the cultural ideal for longer than their earlier developing peers.

\section{Body Composition and Body Image}

In order to investigate the relationship between body composition and body image, Duncan, Woodfield, O'Neill, and Al-Nakeeb (2002) studied 223 (131 males, 92 
females) 11- to 14-year-old English children using a self-report questionnaire to assess body image (Huddy, Nieman, \& Johnson, 1993) and skinfolds to assess adiposity. They found significant inverse relationships between percent body fat and body image scores for males $(r=-.66, p<.01)$, and females $(r=-.62, p<.01)$, analysis of variance showed that the boys had a more positive body image and lower percentage body fat than the girls $(p<.01)$. Similarly, Kimm et al. (1997) found physical appearance to be negatively associated with the sum of triceps, subscapular and suprailiac skinfolds for black and white girls aged 9-10 years $(\mathrm{p}<.01)$.

In contrast, McCabe and Ricciardelli (2003) found weak, but significant, positive relationships between body-image satisfaction and body composition for Australian adolescent boys $(\beta=.20, p<.01)$ and girls $(\beta=.15, p<.05)$ from Grades $7-10$ (mean ages 14.02 years and 13.92 years respectively). However, the explanation for these results may lie in their use of BMI as a measure of body composition. Because BMI is a measure of weight-for-height, rather than actual body composition, an increase in BMI may be due to increased muscle rather than fat, which is in line with the desired sociocultural body image for boys. The positive relationship found for the girls is harder to explain although it may have been as a result of the sporting culture of Australia leading to an increased pressure for girls to aspire to a more muscular body type in a similar manner to the boys. According to the authors, "This may be the case. We certainly find that girls are now attempting to achieve not only a slim body, but also a toned body - not big muscles, but a body that is well toned" (M. McCabe, personal communication, April 11, 2003). Research from England has found conflicting results to those of McCabe and Ricciardelli. In a group of 9-12-year-old girls, Sands and Wardle (2003) reported that in a general factorial analysis of variance there was a significant main effect for age adjusted BMI tertile on body dissatisfaction $\left(F_{(2,305)}=17.02, p<.001\right)$. Furthermore, in a regression analysis, absolute BMI, but not age, was associated with greater body dissatisfaction, explaining $16 \%$ of the variance. This may be an indication of a lesser sporting culture for girls in England compared with Australia although there is no evidence to support such a notion at this stage.

Kolody and Sallis (1995) offered a brief review of the effect of body shape on selfesteem in their introduction to a study of ponderosity and psychological variables in 
children and concluded that findings of previous studies were "conflicting... [and] difficult to reconcile" (p. 1). One reason that they suggested for this conflict is the cross-sectional designs of the previous studies. In contrast, their study was a prospective design involving 314 boys and 253 girls, with a mean age of 9 years, assessed at baseline and one year later. Their findings supported those of previous research that increases in BMI were associated with reductions in body image although the results were significant in girls only. As the authors concluded, although evidence of these associations does not prove the possibility of causal connections between body composition and body image, a prospective study design does add support to such a notion.

Further support is offered from the 3 year study of French, Perry, Leon, and Fulkerson (1996). Cross-sectional analyses found significant negative partial correlations between BMI and physical appearance for both males $(r=-.23, p<.001)$ and females $(r=-.33, p<.001)$. Although partial correlations between changes in physical appearance and BMI over the 3 years were weaker they did show significance for the females $(r=-.11, p<.05)$. Evidence of a relationship between body image and body composition during adolescence is important because if somatotype has an influence on body image it may therefore have an indirect effect on particular activity choices (see Body Image and Behaviour).

\section{Assessment of Body Image}

Physical self-perception instrumentation exists that is able to establish links between physical self-worth and related behaviours (Fox, 1997). Based on the methodology of Harter (1985) and the hierarchical model described above and in Figure 1.2, Fox and Corbin (1989) developed the Physical Self-Perception Profile (PSPP), an instrument that includes scales for assessment of the four sub-domains. This instrument was developed by collecting data from 1,191 university students, mean age 19.7 years, and the authors state that it should not be used for different populations without validity testing and/or modification. However, Whitehead (1995) further developed the PSPP so that it could be used with young people. The Physical Self-Perception Profile for Children is discussed in Chapter 3. 
Questions in the PSPP are structured according to the design of Harter (1982) that aims to minimise social desirability. This 'structured alternative format' presents the respondent with a description of the characteristics of two different people, for example, 'Some people feel uneasy when it comes to doing vigorous physical exercise BUT Other people feel confident when it comes to doing vigorous physical exercise' and the respondent is asked to identify which person is most like them. This legitimises either choice for the participant because it implies that half the people in one's reference group place themselves on one side, whereas the opposite half place themselves on the other. Participants then assess whether the characteristic is 'sort of true for me' or 'really true for me'. This gives a possibility of four different responses to each question. The advantage of this type of question is that it does not have any 'right' or 'wrong' answers, in other words it legitimises any choice of response. Participants are given a mean score, ranging from 1 (low) to 4 (high), on each construct measured.

An alternative to the PSPP is the Physical Self-Description Questionnaire (PSDQ) (Marsh, Richards, Johnson, Roche, \& Tremayne, 1994). Based on a hierarchical model (Marsh \& Redmayne, 1994) similar to that of Fox and Corbin (1989), this instrument is a 70-item questionnaire consisting of 11 scales; including Body Fat, Appearance, General Physical Self-Concept, and Self-Esteem, which closely match those of Body Attractiveness, Physical Self-Esteem, and Global Self-Esteem from the PSPP. Each question is followed by six possible responses ranging from false to true, thus avoiding the structured alternate format favoured by Harter (1985), Fox and Corbin (1989), and Whitehead (1995). In a comparison between the two methods, Marsh et al. (1994) found that the PSPP did not differentiate between factors as well as the PSDQ when completed by Australian high school students. This supports the statement from Fox and Corbin (1989) that the PSPP should not be used, without validation, with populations other than the U.S. university students for whom the instrument was designed.

Marsh et al. (1994) expressed additional concern with the non-standard response scale of the PSPP questions; 23 of the 315 students incorrectly responded to the structured alternate format. They concluded that PSPP responses by young adolescents should be interpreted with caution. This was in contrast to the work by Harter (1982) who used 
the format with children as young as 8 years old. In terms of their use within the current study, the PSPP has been validated with ages 11-15 years (see Chapter 3), more closely matching those present within Project STI, compared with the PSDQ, which has only been validated with ages $12-18$ years.

The stability of body image scores in adolescents has been tested by Duncan et al. (2002). One hundred and twelve participants, aged 12 to 14, completed the Body Image Questionnaire (Huddy et al., 1993) on two occasions 6 weeks apart. The results showed that participants' body image scores were significantly lower on the second occasion $(\mathrm{p}<.001)$, although the test-retest correlation was high $(\mathrm{r}=.97)$. However, these differences were only present in Years 7 and 8 indicating greater stability in the Year 9 (14-year-old) students. It should be noted that the lack of stability seen in the younger participants might be attributed to a lack of understanding of the questions because this questionnaire was developed for use with adults. Use of a questionnaire validated with children may display greater stability.

\section{Body Image and Behaviour}

Unfortunately, no consensus has been reached between researchers regarding the best instrument to use in order to measure body image. Although this makes comparison between results difficult, a number of studies have attempted to establish correlates of behaviour, including body image, using a variety of methods. A project from Taylor et al. (1999) studied 34 African American and Latino girls aged 11 - 15 years old. The girls were organised into six focus groups and discussed their perceptions about physical activity. This study therefore obtained qualitative rather than quantitative data. The core questions asked were open ended, and subsequent probes were designed to target specific areas, for example:

- "Tell me about a time when you had fun doing physical activity." Probe "Tell me reasons why you would want to do exercise. What activities do you like best?" 
- "Tell me about a time when you disliked a physical activity." Probe - "Tell me reasons why you would not want to exercise. What activities do you like least?"

Barriers to physical activity included; dislike of PE classes, inadequate opportunities to participate, and appearance and self-image. Four particular concerns were expressed with reference to the third category:

1. Spoiling of hairstyle and make-up.

2. A fear and dislike of sweating and perspiring.

3. Appearance in the presence of boys.

4. Poor or non-existent shower facilities.

Motivators of physical activity included; fun, social support, and body image and health effects. Discussions regarding the third category noted that the positive effects of exercise included a flat stomach, keeping the right size, losing weight, and keeping good physical and mental health. The results of this study showed that for this small group of girls from minority ethnic backgrounds, issues to do with body image can be both a barrier to and a facilitator of physical activity. Unfortunately no reference was made to sedentary activities and this study used physical activity in the sense of sport rather than less structured activities. However, within the study the commonly used implication was clear, that lowered physical activities lead to increased sedentary behaviours. As previously discussed, this may or may not be the case.

In a study involving African American females, Gordon-Larsen (2001) examined the relationships between knowledge, attitudinal and behavioural factors, and obesity to determine how these factors influence obesity status in urban Philadelphia female adolescents. Obese $(n=32)$ and non-obese $(n=32) 11$ - to 15 -year-old females answered questionnaires and had anthropometric measurements taken. In addition to physical activity (assessed using the 7-day Physical Activity Recall (Sallis, Buono, Roby, Micale, \& Nelson, 1993)) inactivity was also assessed by using the televisionviewing recall survey, whereby all shows on television are listed for each half-hour period of the day and participants select those that they watched. The number of videos watched and hours spent playing video games were also recorded. Results 
showed that obese females spent significantly more time engaged in weekly inactivity then their non-obese peers $(36.16 \pm 14.34$ versus $28.89 \pm 14.98$ hours $)$. When asked to select their own body size and the ideal from a series of nine silhouettes ranging from very thin to obese, there was greater dysphoria for the obese females showing that they were more likely to be dissatisfied with their body size. Unfortunately, it is not possible to directly infer from this that body image and inactivity are related although it does suggest that it may be a worthwhile focus for future research. In contrast to what may have been expected however, there was no significant difference in global self-esteem between the two groups who both exhibited high self-esteem. This was assessed using the Piers-Harris Children's Self-Concept Scale, a self-administered written test with 80 questions requiring 'yes' or 'no' answers, on this scale a high score equates with a high level of self-esteem (Kaplan \& Wadden, 1986).

These results suggested that both groups may have discounted the importance of body image to global self-esteem despite the claims of Harter (1993) and Fox (1997). Maturational status was assessed by recording day, month, and year of menarche, and no significant difference was found between groups. Unfortunately no separate analysis was done between time since menarche and level of inactivity or body image.

Hanley et al. (2000) carried out a similar study of overweight and associated behavioural factors. Height and weight were measured and fitness level, television viewing, body image concepts, and dietary intake were assessed in 242 young people aged $10-19$ years (boys and girls). Television viewing was assessed by self-reported usual hours of viewing, including video games and movies, per day. To assess body image participants were asked to identify their own body build, the body build they would like to achieve in the future, and the healthiest somatotype for men and women, from a series of nine somatotype drawings. No assessment of maturational status was reported.

In support of the findings of Gordon-Larsen (2001), an association was observed between obesity and television watching with a 2.5 -fold risk of overweight in participants who watched $\geq 5$ hours/day compared with those who watched $0-2$ hours/day (OR $=2.52 ; 95 \% \mathrm{CI}: 1.06,5.98)$. Overweight females were more likely to 
choose a smaller ideal body image than non-overweight females. Although these results were not shown to be significant, they did reflect the increased dysphoria reported by Gordon-Larsen. No such pattern was shown for males, which suggests that the relationship between inactivity and body image may be weaker for males

Body image of young people reaching the end of adolescence was researched by Van den Bulk (2000). This survey of 1035 Flemish 17- and 18-year-olds aimed to assess the relationship of television watching (no other sedentary behaviours) with a variety of health behaviours and outcomes including body satisfaction. Participants were asked to provide six pieces of information regarding body assessment:

1. Their current weight and height, from which BMI was calculated.

2. How happy they were with their bodies on a 5-point scale.

3. Their preferred height and weight, from which an ideal BMI (IBMI) was calculated. BMI - IBMI was also calculated.

4. A rating of their looks on an 11-point scale.

5. An assessment of the importance of looks for them, also on an 11-point scale.

6. Whether or not they were on a diet.

A very small but significant relationship was found between dissatisfaction with current weight and volume of TV viewing for males only $(r=.15, p<.01)$, for females a small but significant relationship was found between total viewing volume and BMI - IBMI $(r=.16, p<.01)$. All other relationships were non-significant.

Because this was not a longitudinal study, conclusions regarding cause and effect cannot be made. For example, dissatisfaction with weight or BMI could have been caused by exposure to 'ideal body types' from television programmes, rather than being the cause of more television viewing. In addition, the use of self-reported measures of height and weight has been brought into question with this population (Brener, McManus, Galuska, Lowry, \& Wechsler, 2003; Crawley \& Portides, 1995; Goodman, Hinden, \& Khandelwal, 2000; Hill \& Roberts, 1998). 
Stronger evidence was offered by Kolody and Sallis (1995) in their one year prospective study that was discussed earlier. In addition to their findings of a negative relationship between BMI change and body image they also reported that, for overweight boys, increases in BMI were associated with an increased preference for watching television compared with walking or playing a running game with friends.

\section{Behavioural Choice Theory}

One approach to explaining adolescents' activity choices is Behavioural Choice Theory (BCT). This theory "explicitly recognizes that preference for any given reinforcer [behaviour] arises within a context involving other reinforcers and associated environmental constraints, and that consideration of this context is critical to an analysis of the determinants of reinforcement value" (Vuchinich \& Tucker, 1988, p.182).

Epstein (1998) discussed how BCT has the potential to develop a greater understanding of the factors influencing the allocation of an individual's time. He stated that four general principles of BCT can be applied to studies of sedentary and active behaviours:

- Firstly, choice of a particular behaviour depends on its cost and the cost of any alternatives, in other words, the behavioural cost of being sedentary versus the behavioural cost of being more active.

- Secondly, the reinforcing value of a behaviour depends partly on the availability of any alternatives.

- Thirdly, in order to motivate people to obtain a reinforcer then the availability of choice is important. In other words, people are more likely to continue in a behaviour that they themselves have chosen to participate in.

- Finally, choice depends partly on the time delay between choosing the behaviour and receiving the value of the reinforcement. For example, the benefits of sedentary behaviour (e.g., entertainment) may be immediate whereas the benefits of physical activity (e.g., physical fitness) may be delayed. 
For adolescents experiencing the changing body composition of puberty, physically active behaviours may have a higher cost of reduced physical self-esteem compared with more sedentary behaviours. For instance, in the study of Taylor et al. (1999) examples of barriers to physical activity included appearance and self-image; in particular, spoiling of hairstyle and make-up, a fear and dislike of sweating and perspiring, and appearance in the presence of boys. The current study, therefore, hypothesises that reductions in body image associated with body compositional changes may, in turn, be associated with increases in sedentary behaviour as participants seek to minimise reductions in self-esteem.

\section{Summary of Psychological Factors}

- A relationship exists between physical self-worth and global self-esteem.

- Body image is a sub-domain of physical self-worth and instruments exist that are able to quantify relationships.

- It may be difficult for individuals to discount their physical appearance due to cultural pressures.

- Females find body image to be more important than males, although both genders find 'looks' to be important.

- A negative relationship exists between body image and body fat.

- Body image declines as age and maturity increases for females; the opposite may be true for males.

- The development of body image may be associated with timing of maturation.

- Stability of body image may be less in younger adolescents.

- Body image can have a positive or negative effect on physical activity but reported links between body image and sedentary behaviour require further research.

- According to behavioural choice theory, adolescents' behaviour choices may change according to their psychological cost and the costs of any alternatives.

One point that is noticeable from this review of literature is the large variety of different methods and models being used to assess self-esteem/body image, thus 
making it difficult to make meaningful comparisons between studies. A more uniform use of measurement tools would be a positive development. 


\section{Part 4. Sleep and Adolescence}

An additional factor that may have a confounding effect on the total time spent in sedentary behaviours is the amount and timing of sleep. During data entry for the current study it became apparent that older participants were completing a greater amount of the daily diary as younger participants went to bed earlier. It may therefore be possible that a simple comparison between age or pubertal groups on time spent being sedentary does not compare 'like with like'. This is because individuals who go to bed later have more time available in the evening for behaviours that are more likely, at that time of day, to be sedentary. In contrast, those who get up earlier in the mornings, especially at weekends, may have more time to be either active or sedentary.

This pattern of sleeping is supported by research that has shown weekday and weekend day bedtimes to be delayed progressively with age (Wolfson \& Carskadon, 1998). Weekday wake-up times are more consistent (Wolfson \& Carskadon, 1998), whilst weekend wake-up times are increased with both age and pubertal status (Laberge et al., 2001). According to Laberge et al. (2001), "puberty influences the timing of sleep patterns in early adolescents. Physiological concomitants of puberty may thus have an effect on the circadian phase delay of the sleep-wake cycle characteristic of adolescence" (p.64).

An alternative hypothesis to that posited above, therefore, is that increases in sedentary time for older or more developed individuals may be associated with increases in evening time spent awake, during which more active alternative behaviours are not available. In order to obtain a picture of behaviour change that is associated with changes in the relative behavioural costs of the behaviours to the participant rather than to the availability of suitable time, it may be necessary to control for the amount of sleep when comparing total sedentary times between different ages or pubertal stages. 


\section{Chapter 1 Conclusions}

Overweight and obese adolescents have more chance of becoming overweight adults (Guo \& Chumlea, 1999) with all the resulting health problems that can occur. In order to help reduce the chance of becoming overweight, alterations must be made to an individual's energy equation i.e. energy expenditure must increase or energy intake must fall. Energy expenditure may be increased by one of two methods, an increase in active behaviours or a decrease in sedentary behaviours. Understanding the determinants of different behaviour types will help researchers design interventions that can achieve such changes.

Results of previous studies have suggested that sedentary and active behaviours are separate and distinct constructs (Cameron et al., 2003; Dietz, 1996; Gordon-Larsen et al., 2000; Marshall et al., 2002; Raitakari et al., 1994; Salmon et al., 2000; Schmitz et al., 2002). Therefore, in order to understand the determinants of sedentary behaviours, data analysis should be based on direct assessments of such behaviours rather than from assumptions made from reported levels of more active behaviours. However, the degree of success of such research will depend on using a measurement instrument that is capable of accounting for all free-time activity within the day because assumptions regarding unreported time may not be valid. Previous studies of inactivity have produced equivocal results possibly due to measures of behaviour that were too limited. A suitable method of data collection, therefore, is one that allows the recording of all sedentary behaviours as defined by Ainsworth et al. (2000) and Owen et al. (2000).

Future studies should include measures of maturational status and body composition, as well as psychological profiles, such as body image, to allow associations with inactivity to be assessed. In addition, sleep time should be controlled for during analysis because it may affect the total amount of time available without being a free choice behaviour. 


\section{Chapter 2: Study Design}

The current study was designed and carried out in order to address some of the issues arising from the review of literature described in Chapter 1 . That is, it was a longitudinal study that focused on and assessed a range of sedentary behaviours based on self-report of all behaviours throughout the day, and maturational status and body composition were taken into account. It was hoped that a study of sedentary behaviour change within adolescence would help to explain if and why such behaviour changes occur at this time.

The study lasted for 12 months and was divided into three phases. Phase 1 took place in March - June 2002 and formed the baseline data for the study. Phase 2 took place in October - December 2002, and Phase 3 took place in March - May 2003. A longitudinal study design was employed because stronger evidence for a pubertalchange effect could be offered by within-participant changes rather than betweenparticipant differences. Cross-sectional results from Phase 1 were used to inform the analysis of the longitudinal data, which assessed changes from Phase 1 to Phase 3.

In order to observe changes in maturity that encompassed the complete range of pubertal stages within the twelve months available a mixed-longitudinal design was used. This meant starting the study with three cohorts that included participants who could be expected to be at different pubertal stages at the first assessment. In practical terms this meant using school year groups whose students were aged $10-14$ years at Phase 1. School Year Groups 6, 8, and 10 were chosen for this purpose. Because schools in the UK do not include the range of year groups that were needed to complete the whole study it was decided to use two schools that were associated with each other so that as students completed high school they could be more easily tracked into the upper school.

Phases were chosen to be at six-month intervals because expected changes in BMI within this period could be measured with an acceptable level of power $(\alpha=.05,1-\beta=$ $.80)$ and with a number of participants that was not prohibitive ( $\mathrm{n}<200$, see the power 
calculation in Chapter 5). It was also considered that changes in pubertal level would be seen for some participants within this time scale. However, during the analysis it became apparent that six months was not long enough for sufficient pubertal changes to occur and so the primary analysis was modified to observe changes over the twelve-month period between Phases 1 and 3.

Assessments were school based with participants informed of their appointments on the preceding day. The methods considered for use are described in Chapter 3 and those chosen are described in Chapter 4. The Gantt chart in Figure 2.1 shows the timetable for the complete study. 


\section{Chapter 3: Methods Review}

\section{Assessment of Sedentary Time}

Measuring physical activity and inactivity is notoriously difficult and numerous methods and appliances have been tried including, for example, pedometers, accelerometers, actometers, electronic load transducers, and foot-contact monitors (Zhang, Werner, Sun, Pi-Sunyer, \& Boozer, 2003). The current 'gold standard' for activity assessment is the doubly-labelled-water method that can provide a reliable measure of energy expenditure associated with physical activity over periods of $1-3$ weeks (Bouten, Verboeket-van de Venne, Westerterp, Verduin, \& Janssen, 1996). This method is limited to studies of small populations due to the need for sophisticated analysis and its high cost (1996 price $\sim \$ 500$ (approximately $£ 300$ ) per person (Bouten et al., 1996)). Additionally, this method only indicates the average level of daily energy expenditure and does not provide information about patterns of behaviour or levels of inactivity.

Pedometers and accelerometers have been particularly popular in recent studies after Wong et al. (1981) developed a portable accelerometer for measuring human energy expenditure. This device used a ceramic transducer that produced an electric charge due to stylus displacement during movement. The accelerometer was then calibrated, by comparing the meter reading with oxygen uptake measurements, so that it could give a measure of energy expenditure.

Further technological developments have led to the production of accelerometers such as the CSA 7164 from Computer Science and Applications, Inc. [CSA], Shalimar, FL. This device allows both duration and intensity of active and inactive behaviours to be recorded and downloaded to a PC. The use of such accelerometers with children has been validated in studies that have compared their use against heart-rate telemetry (Janz, 1994) and indirect calorimetry (Trost et al., 1998). However, the cost may be prohibitive to large-scale studies, the system set-up kit costs $\$ 929$ with additional monitors costing $\$ 400$ each. 
A recent innovation has been the Intelligent Device for Energy Expenditure and Activity (IDEEA) (Zhang et al., 2003). This device requires the placement of five sensors on the sternum, upper legs, and inferior side of the feet. The advantage of this new system is its ability to differentiate between 32 types of activity including five basic gaits, 5 primary and 22 secondary postures, five limb movements and five transitions. Zhang et al. validated the IDEEA on 76 participants ranging in age from 13 to 72 years and found that correct identification rates averaged $98.9 \%$ for posture and limb movement type and $98.5 \%$ for gait type. The major disadvantage of this system is the reliance on, and inconvenience of, the correct placement of all five sensors. The cost may also, once again, be prohibitive, each complete system including the recorder, interface, battery charger, and download and data processing software costs $\$ 4,950$.

Unfortunately, devices such as the IDEEA or accelerometers do not allow differentiation of activities of similar intensity or type but with different behavioural determinants. For example, the current project assessed free-time behaviours but would have been unable to distinguish between those were free-time and free-choice, such as reading a book or writing to a friend, and those that were not, such as completing homework, if such devices were used.

Alternative methods of assessing activity and inactivity patterns in free-living individuals include questionnaires and diaries/logs that require the recall of behaviour, usually over a period of one day or one week. One of the main problems with using this type of instrument with adolescents is the burden that it places on participants and the issue of compliance although this is likely to vary with the population under study, number of days record-keeping, and other factors (Sallis, 1991).

Despite concerns regarding errors due to (a) erroneous recall of the frequency and duration of the activities, and (b) poor estimation of the intensity of the activity (Montoye et al., 1983), activity diaries are considered one of the most accurate techniques for obtaining subjective activity data from adults, although results from children may be less valid (Sirard \& Pate, 2001). However, Sallis (1991) reported that, for children, diary measures have the strongest validity data compared with other self- 
report measures such as self-administered recall, interview administered recall or proxy reports by parents or teachers.

In addition, Sallis and Owen (1996) suggested that because the influences of physical activity are not well understood, then physical activity research may benefit from the application of social ecological models, these focus attention on the social, institutional, and cultural contexts of people-environment relations (Stokols, 1992) and assume that these factors interact with each other rather than acting in isolation to affect an individual's behaviour. Applying a similar approach to the study of adolescents' inactivity patterns, it is necessary to study a variety of determinants within the environment that affect behavioural patterns and choices in order to fully understand how these choices are made. With specific reference to children and adolescents Sallis et al. (1992) have established that activity choices have a number of influences that include:

Physical environment factors; for example, access to physical activity facilities and programmes, time spent outdoors.

Biological and developmental factors; for example, genetics, fitness, puberty, body composition.

Psychological factors; for example, self-efficacy, perceptions of barriers to participation.

Social and cultural factors; for example, socio-economic status, race and ethnicity, parental and peer influences.

Diaries are particularly useful for the collection of such data. Also, for the current project intensity of activities was not important because the behaviours of interest were considered to be inactive and of minimum intensity. Activity diaries were thus a valid and cheap tool for the assessment of adolescents' inactivity and ideal for use within Project STIL. Their use within the study is described in greater detail below. 


\section{Free-Time Behaviour Diary}

The pilot study to the current project used momentary time sampling (MTS) diaries in order to assess adolescents' free-time behaviour, both active and inactive (Marshall, 2001). The diaries used in the study required students to note their primary and secondary activities every 15 minutes from $7 \mathrm{am}$ until midnight exclusive of time spent in school. All students were required to complete the diary for three weekdays and one weekend day as this had been shown to be the minimum number of days required to represent habitual behaviour. Each behaviour was assumed by the diary to have lasted for 15-minutes, and placed by researchers into one of 22 categories (see Table 3.1). These had been inductively derived from the raw interval-level descriptions of young peoples' self-reported behaviour. Total time spent in each category was then calculated.

In addition to a short description of the primary and secondary activities they were involved with, each student indicated (via a choice of numbered options) where they

Table 3.1. Categories used for coding behaviours reported within the diaries.

\begin{tabular}{ll}
\hline 1. Sleeping & 12. Listening to music \\
2. Personal Care & 13. Telephone use \\
3. Eating/drinking & 14. Homework \\
4. At school & 15. Reading \\
5. Motorised transport & 16. Active hobbies \\
6. Active transport & 17. Cognitive hobbies \\
7. TV/film watching & 18. Unstructured play \\
8. Computer use & 19. Chores \\
9. Computer gaming & 20. Work \\
10. Talking & 21. Sports \\
11. 'Hanging out' & 22. Other \\
\hline
\end{tabular}


were, who they were with, why they were doing the activity, current mood status, and current energy level. Before starting the diary, participants also provided general information about themselves and at the start and end of each day they were asked to make short comments about temperature, weather, and activity undertaken whilst at school. The current study used an adapted version of the diary used in the pilot that did not require information regarding secondary activities, why they were doing the activity, current mood status, and current energy level, as analysis had shown that these were not significant predictors of sedentary behaviour (Marshall, 2001).

It was necessary to assess the reliability of the information recorded by the pilot study diaries to show that they were representative of the students' actual time spent in a variety of behaviours. In order to do this a real-time diary (RT) was used in a reliability study as reported by Marshall (2001) but which is described in greater detail below.

\section{Reliability Testing of the Free-Time Diary}

\section{- Participants}

Students were sampled from local schools. One class of Year 8 students $(N=30)$ and one class of Year 9 students $(N=29)$, from school (A) were given a diary each. Volunteers from Year $10(\mathrm{~N}=30)$ were given a diary each in school (B). An additional set of Year $8(\mathrm{~N}=6)$ and $9(\mathrm{~N}=3)$ students were given a diary each in school (C). All students were aged between 12 and 16 years.

\section{- Method}

Participants were given a package containing a child-parent consent form, a set of instructions for students and a set for parents, a student RT activity diary (randomly assigned either a week day or weekend day) and a parent validation diary. The RT diaries provided a self-report measure of free-time behaviour that accounted for every minute of the day outside of school hours. 
The diary ran from 7.00 a.m. to 9.00 a.m. and 3.00 p.m. to midnight (weekday) or 7.00 a.m. to midnight (weekend day), in one-minute increments. Students were required to indicate the start and finish of each activity during the day with all minutes accounted for and no overlap between activities. Only the primary activity at any one time needed to be recorded (see Figure 3.1). It was stressed to the students that every activity that lasted for longer than a minute needed to be recorded, including using the bathroom, cleaning teeth etc.

Figure 3.1. Recording the first activities of the day.

\begin{tabular}{|c|c|c|}
\hline Time & $\begin{array}{l}\text { Start/stop } \\
\text { indicator }\end{array}$ & $\begin{array}{l}\text { Description of what you are doing } \\
\text { e.gs, sleeping, eating, doing homework, talking with friends, watching TV, } \\
\text { listening to music, on telephone, walking to school, etc. }\end{array}$ \\
\hline \multicolumn{3}{|c|}{$\frac{1}{7.00 \mathrm{am}}$} \\
\hline \multicolumn{3}{|l|}{.01} \\
\hline \multicolumn{3}{|l|}{.02} \\
\hline \multirow{2}{*}{\multicolumn{3}{|c|}{ Shaping }} \\
\hline & & \\
\hline \multicolumn{3}{|l|}{.05} \\
\hline \multicolumn{3}{|l|}{.06} \\
\hline \multicolumn{3}{|l|}{.07} \\
\hline \multicolumn{3}{|l|}{.08} \\
\hline \multirow{2}{*}{\multicolumn{3}{|c|}{ Gotting droend }} \\
\hline .10 & & \\
\hline .11 & $\downarrow$ & \\
\hline
\end{tabular}

In addition to recording each activity, between 7.00 p.m. and 10.00 p.m., every 15 minutes, two additional questions had to be answered (see Figure 3.2).

Figure 3.2. Examples of students' additional questions.

\begin{tabular}{|l|l|}
\hline At 7.00pm: & $\begin{array}{l}\text { Who's with you? } \\
\text { (circle one number) }\end{array}$ \\
\hline $\begin{array}{l}\text { Where are you? } \\
\text { (ircle one number) }\end{array}$ & \\
\hline $1=$ My bedroom & \\
2 = Living room & $1=$ I'm alone \\
3 = Kitchen & $2=$ Friends \\
4 = Bathroom & $3=$ Family \\
$5=$ Other room & \\
$6=$ Friend's house & \\
$7=$ In town & \\
$8=$ At school & \\
$9=$ In car, bus or taxi & \\
$10=$ Other (describe) & \\
\hline
\end{tabular}

For the same 15 -minute intervals between 7.00 p.m. and 10.00 p.m., a parent/carer of each student completed a diary that recorded what, in their opinion, their child was 
doing, where they were, and who they were with. If the parents did not know any of the information they were asked to write this. There was also a question to indicate the degree of certainty that the parent had in their answers $(1=$ not at all confident, $2=$ somewhat confident, 3 = very confident). Part of the Parent Observation Diary is shown in Figure 3.3.

Figure 3.3. Part of the Parent Observation Diary

\begin{tabular}{|c|c|c|}
\hline & Question 1 & \\
\hline Time & $\begin{array}{l}\text { What is your child doing at this exact } \\
\text { time? } \\
\text { (Write activity) } \\
\text { e.g., sleeping, eating, doing homework, talking with } \\
\text { friends, watching TV, listening to music, on telephone, } \\
\text { walking to school, etc. }\end{array}$ & $\begin{array}{l}\text { How confident } \\
\text { are you that this } \\
\text { is correct? } \\
\text { (please circle one) } \\
1=\text { not at all confident } \\
2=\text { somewhat confident } \\
3=\text { very confident }\end{array}$ \\
\hline $8.00 \mathrm{pn}$ & 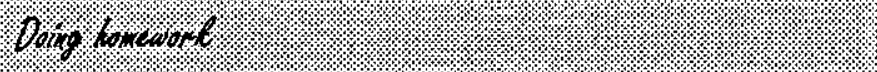 & 2. \\
\hline
\end{tabular}

- Returned Data

From 96 diaries given out, 37 were returned representing a $37.8 \%$ return rate. The number of returns in each age group was as follows:

$$
\begin{aligned}
& \text { Year } 8=11 \\
& \text { Year } 9=11 \\
& \text { Year } 10=15 \\
& \text { Parents' observation }=34
\end{aligned}
$$

\section{- Data Analysis}

Data was analysed using the statistics package SPSS v11.0. Intra-class correlation coefficients were calculated to assess reliability of using a 15-minute or 30-minute sampling frame. Pearson correlation coefficients were used to evaluate the correlations between students' and parents' reports. 


\section{a) Diary Reliability Data}

The purpose of this analysis was to evaluate whether or not a momentary time sampling method correlated closely enough with the real-time diary for MTS to be considered a reliable method of measuring students' free-time behaviour.

When recording the data returned from the diaries, each reported activity was placed into one of the categories in Table 3.1. When analysing the RT data, the number of minutes spent on each reported activity was counted (see Figure 3.4), and the total time spent in each of the 22 behaviour types was calculated and recorded (e.g., sleep_r). These results were then compared to those that would have been obtained if each student had been asked to record their activity using an MTS approach, noting their behaviour every 15 minutes. In order to make this comparison, a snapshot was taken on each hour, quarter past each hour, half past each hour, and quarter to each hour (see Figure 3.5), this method makes the assumption that each behaviour lasts for the whole 15-minute block. The total number of 15-minute blocks of behaviour and, therefore, total estimated time spent in each behaviour type was then calculated and recorded (e.g., sleep_d).

Figure 3.4. Calculating the actual time spent in each activity.

\begin{tabular}{|c|c|c|}
\hline Time & $\begin{array}{l}\text { Start/stop } \\
\text { indicator }\end{array}$ & $\begin{array}{l}\text { Description of what you are doing } \\
\text { e.g, sleeping, eating, doing homework, talking with friends, watching TV, } \\
\text { listening to music, on telephone, walking to school, etc. }\end{array}$ \\
\hline \multirow{2}{*}{\multicolumn{3}{|c|}{ 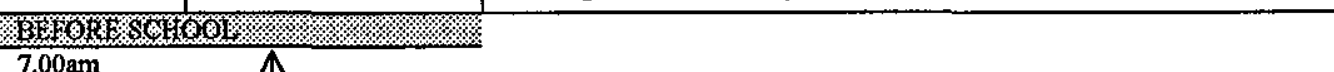 }} \\
\hline & & \\
\hline \multicolumn{3}{|c|}{.01} \\
\hline \multicolumn{3}{|l|}{.02} \\
\hline \multicolumn{3}{|l|}{.03} \\
\hline \multicolumn{3}{|l|}{.04} \\
\hline \multicolumn{3}{|l|}{.05} \\
\hline \multicolumn{3}{|l|}{.06} \\
\hline \multirow{2}{*}{\multicolumn{3}{|c|}{$\frac{.01}{.08}$}} \\
\hline & & \\
\hline \multicolumn{3}{|r|}{ Geting dromedd } \\
\hline \multicolumn{3}{|l|}{.10} \\
\hline .11 & & \\
\hline
\end{tabular}


Figure 3.5. Taking 15-minute snapshots of activity.

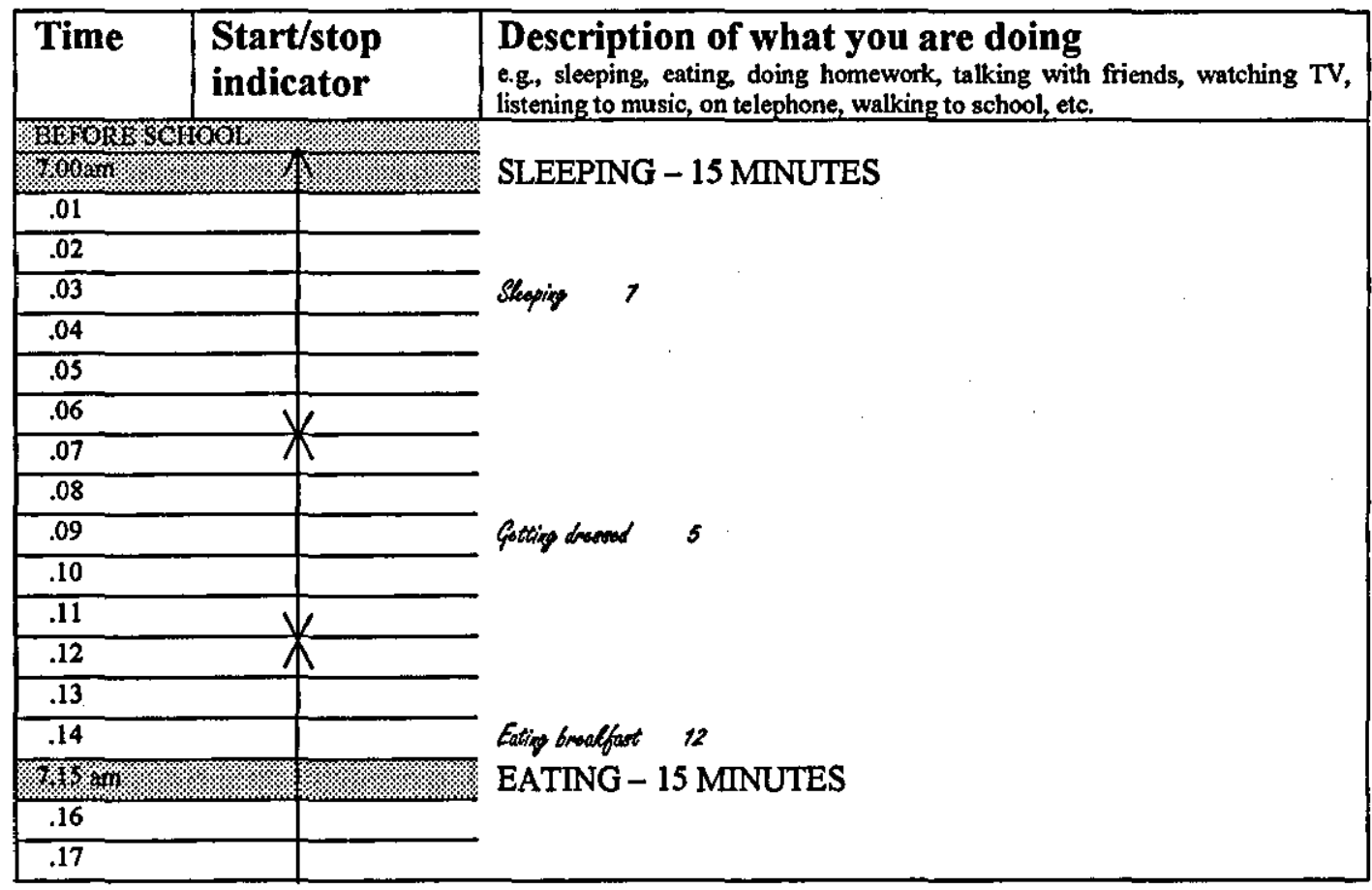

Finally, snapshots were taken every half hour and correlated with the RT data to assess whether a 30-minute sampling frame could be used as an alternative to the 15minute frame. Intraclass correlation coefficients (ICCs) were calculated for each pair of behaviours e.g., sleep_r versus sleep_d. Intraclass correlations are a measure of reliability that indicate the degree of agreement between the ratings provided by multiple judges (Lahey, Downey, \& Saal, 1983). The type of ICC used was a two way mixed model because the rater factor (the MTS versus RT diary) was fixed and both diaries were used to rate all participants (Shrout \& Fleiss, 1979). An absolute level of agreement was used because any systematic differences between the diaries were considered important (Nichols, 1998); this level of agreement includes the variance between measures within the denominator of the ICC (McGraw \& Wong, 1996).

All correlations included only observations where min/day for the RT diary were greater than zero. ICCs were first calculated for RT versus MTS (15-minute) data (see Table 3.2), followed by RT versus MTS (30-minute) data (see Table 3.3). 


\section{b) Parent-child validity data}

The purpose of this analysis was to assess the validity of the diary to accurately record behaviour by comparing the students' reports with those of their parents.

Each student's reported activity along with the information about where they were and whom they were with (see Figure 3.2) was compared with the information recorded by their parents (see Figure 3.3). As previously, each activity was categorised according to Table 3.1, additional categories 99 (missing data) and 23 (parent not aware of behaviour) were included that could be omitted from the calculations. This avoided any correlations from showing that students misreported their activity when in fact it was the parents who were unable to corroborate the information. For each parent-child pairing, the number of agreements between the following variables were calculated:

- Parent's opinion of activity (p_act) / child's opinion of activity (c_act),

- Parent's opinion of where the child is (p_place)/ child's opinion of where he/she is (c_place),

- Parent's opinion of who the child is with (p_who) / child's opinion of who he/she is with (c_who),

This was followed by the number of agreements as a percentage of the number of intervals for each student (\% Activity agreement; \% Place agreement; \% Who agreement). The mean number of percentage agreements was then calculated and can be seen in Table 3.4 .

Finally, Pearson correlation coefficients were calculated between the percentage number of agreements and the mean value of each parent's confidence in the accuracy of their answers. These results can be seen in Table 3.5. 
The ICCs shown in Table 3.2, for the RT diary versus 15-minute MTS, were all high (intraclass $r>.90$ ) with the exception of telephone use (intraclass $r=.36$ ). The ICCs shown in Table 3.3, for the RT diary versus 30-minute MTS, were all within the range 0.70-1.00 with the exception of telephone use, which was not calculated.

Percentage agreements between parent and child reported behaviour, shown in Table 3.4, were high for where the child was (\% Place Agreement), and whom the child was with (\% Who Agreement), both $81 \%$. Agreement about what behaviour the child was involved with (\% Activity Agreement) was lower at only $70 \%$.

Table 3.2. Intra-class correlations and $95 \%$ confidence intervals between behaviour types, 15-minute sampling frame.

\begin{tabular}{rcccc}
\hline \multicolumn{1}{c}{ Activity } & $\mathrm{N}$ & ICC & Lower Limit & Upper Limit \\
\hline Personal Care & 34 & .91 & .83 & .95 \\
Eating/drinking & 36 & .94 & .88 & .97 \\
Motorised transport & 17 & 1.00 & .99 & 1.00 \\
Active transport & 20 & .93 & .83 & .97 \\
Watching tv/video & 32 & .99 & .98 & 1.00 \\
Computer use & 13 & .99 & .98 & 1.00 \\
Computer games & 7 & .98 & .91 & 1.00 \\
Talking to & 12 & .98 & .95 & 1.00 \\
friends/family & 16 & .99 & .98 & 1.00 \\
Shopping/hanging out & 16 & .97 & .90 & .99 \\
Listening to music & 19 & .36 & .00 & .84 \\
Using the phone & 7 & .98 & .94 & .99 \\
Homework & 17 & .96 & .80 & .99 \\
Reading & 9 & .98 & .93 & 1.00 \\
Active hobbies & 9 & .98 & .38 & 1.00 \\
Cognitive hobbies & 5 & 1.00 & .98 & 1.00 \\
Unstructured play & 8 & .99 & .98 & 1.00 \\
Chores & 13 & .99 & .93 & 1.00 \\
Paid work & 4 & 1.00 & .99 & 1.00 \\
Sports/exercises & 16 & & &
\end{tabular}


Generally parents were highly confident in their answers; the mean levels of confidence were: activity, 2.7; place, 2.8; and whom with, 2.9. However, Pearson correlation coefficients between percentage agreements and parent's confidence in their answers was low (see Table 3.5), ranging from $r=-.055$ to $r=.200$.

Table 3.3. Intra-class correlations and $95 \%$ confidence intervals between behaviour types, 30-minute sampling frame.

\begin{tabular}{rcccc}
\hline \multicolumn{1}{c}{ Activity } & $\mathrm{N}$ & ICC & Lower Limit & Upper Limit \\
\hline Personal Care & 31 & .70 & .45 & .84 \\
Eating/drinking & 30 & .79 & .60 & .90 \\
Motorised transport & 15 & .97 & .92 & .99 \\
Active transport & 19 & .87 & .70 & .95 \\
Watching tv/video & 32 & .96 & .92 & .98 \\
Computer use & 13 & .98 & .93 & .99 \\
Computer games & 6 & .78 & .04 & .97 \\
Talking to & 8 & .93 & .70 & .99 \\
friends/family & 13 & .99 & .96 & 1.00 \\
Shopping/hanging out & 13 & .68 & .96 \\
Listening to music & 16 & .88 & - & - \\
Using the phone & 2 & - & .80 & .97 \\
Homework & 15 & .93 & .60 & .99 \\
Reading & 8 & .93 & .70 & .98 \\
Active hobbies & 8 & .93 & .11 & .99 \\
Cognitive hobbies & 5 & .92 & .95 & 1.00 \\
Unstructured play & 6 & .99 & .84 & .99 \\
Chores & 11 & .95 & .88 & 1.00 \\
Paid work & 4 & .99 & .99 & 1.00 \\
\hline Sports/exercises & 15 & 1.00 & & \\
\hline & & & & \\
\hline & & & & \\
\hline
\end{tabular}

Table 3.4. The mean percentage agreements between parent and child reported behaviour.

\begin{tabular}{rccc}
\hline & $\mathrm{N}$ & Mean & Std. Deviation \\
\hline \% Activity Agreement & 29 & 69.5976 & 30.1482 \\
\% Place Agreement & 31 & 80.5136 & 23.7173 \\
\% Who Agreement & 32 & 80.7255 & 24.8503 \\
\hline
\end{tabular}


Table 3.5. Pearson correlations between $\%$ agreements and parent's confidence in their answers.

\begin{tabular}{rccc}
\hline & $\mathrm{N}$ & Correlation & Sig. (2-tailed) \\
\hline \% Activity Agreement & 28 & .200 & .308 \\
\% Place Agreement & 31 & .249 & .177 \\
\% Who Agreement & 32 & -.055 & .764 \\
\hline
\end{tabular}

\section{- Discussion}

For the calculation of intra-class correlations (and 95\% confidence intervals) between the real-time diaries and momentary time sampling a two-way mixed model of analysis was chosen with an absolute level of agreement. This was in order to assess the degree to which the two methods were measuring identical amounts of time spent in different behaviours rather than just the correlation between them. This calculation will highlight any bias inherent in one diary compared to the other. The precision of these ICC estimates is uncertain because of the low numbers of reported instances for many of the behaviours. However in general the ICCs from 15-minute MTS were high, with the exception of telephone use $(\mathrm{ICC}=.36)$.

Momentary time sampling was first suggested as a method for recording behaviour by Bindra and Blond (1958). Rather than making a continuous record of all behaviours of the participant, only the behaviour that occurs precisely at the start of an $x$-minute interval is noted. MTS may be used for two different purposes (Brulle \& Repp, 1984); firstly, to create time series data in which patterns of behaviour are tracked across a sampling period, secondly, to calculate the average duration of behaviour across a sampling period by making the assumption that the recorded behaviour lasted for the entire duration of the interval. The MTS diary used in the current study was based on the second of these methods and worked by assuming that over a period of time discrepancies between MTS and RT will even out. For example, if eating takes 20 minutes but is only recorded once in an MTS frame then it will be recorded as a 15 minute activity and will be 5 minutes short. However on another occasion eating may only take 10 minutes but still be recorded once and therefore still be recorded as a 15 - 
minute activity. With enough recording occasions the differences even out and correlations increase.

Harrop and Daniels (1985) (using much shorter time periods and behaviours, $10-30$ s) suggested that the accuracy of MTS may be increased for longer duration behaviours and higher rate behaviours. Therefore, the low correlation seen for telephone use may have been due to the particularly short duration of the activity coupled with low frequency of the behaviour because over a period of time the discrepancy between MTS and RT did not have a chance to even out. Larger numbers of participants may have increased some of the low correlations. Overall it was considered that the results showed enough high correlations for the 15-minute momentary time sampling diary to be considered a reliable recording tool for measuring time spent in a variety of behaviours.

Intra-class correlations between the real-time diaries and 30-minute momentary time sampling, although high, were lower than for the 15-minute MTS. Thirty-minute sampling was thus discarded as having lower reliability.

Although the percentage agreements between parent and child were high, agreement on activity was noticeably lower than the other two (70\% versus $81 \%$ ). Closer inspection of the raw data where discrepancies existed showed that although the parents were often aware of the where the child was (e.g., in their bedroom), they were not usually with the child and therefore were unable to correctly describe the child's activity. The same reasoning may be applied to the low Pearson correlations obtained between percentage agreements and parental confidence in their answers (see Table 3.5). Inspection of the raw data showed that many parents rated the confidence in their answers as 3 (maximum) even if their child was not with them (or even in the house) at the time.

With the diaries used in this study the students wrote down their behaviour and researchers translated this information so that the behaviour could be classified according to its intensity and type. This process required certain assumptions regarding the behaviour because the researcher did not know exactly how intense the behaviour was. For example, a student who wrote down 'walking to school' had this 
activity classified as 'active transport' although no differentiation was made between fast and slow walking. This subjective assessment of activity intensity is one of the negative aspects of using this kind of measuring tool.

One advantage of using the diary is that it can be used to collect demographic information alongside the data regarding behaviour. Therefore, before the first day of data collection the participant completes three pages of questions regarding:

- Age.

- Home postal code (socio-economic status).

- Race and religion.

- Pubertal Status.

- Family details.

- Birth details.

- Size and position of home and distance from school.

- Availability of objects that promote inactive (e.g., televisions, video game players) or active (e.g., bicycles, roller skates) behaviours around the home.

Thus, associations between inactivity and the biological and developmental, and physical environment factors, as suggested by Sallis et al. (1992), can be calculated.

\section{- Conclusion}

Fifteen-minute MTS was a reliable method for assessing total time spent in a range of behaviours compared with RT minute-by-minute assessment. A 15-minute MTS diary was therefore considered to be suitable for assessment of total sedentary time within the current study.

Compared with other methods of assessing free-living behaviour patterns, diaries are a useful instrument when large numbers of participants need to be studied because they are cheap to produce and easy to distribute. They are also good at collecting information on a number of variables at once. For example, information about the participant, family, socio-economic status, as well as details about behaviour can all 
be included within the diary. However, the burden can be very high, and assumptions have to be made regarding certain aspects of each recorded behaviour including the intensity.

\section{Assessment of Body Composition}

\section{Body Mass Index}

\section{a) BMI in Adults}

Reference points for optimum BMI in adults are $18.7-23.8 \mathrm{~kg} / \mathrm{m}^{2}$ for women, and $20.1-25.0 \mathrm{~kg} / \mathrm{m}^{2}$ for men. These values were rounded to $20-25 \mathrm{~kg} / \mathrm{m}^{2}$ for both sexes by Garrow (1982). The setting of these limits is related to the relationship of BMI to morbidity and mortality. This relationship is a J-shaped curve with its lowest point between the range $20-25 \mathrm{~kg} / \mathrm{m}^{2}$. Below $20 \mathrm{~kg} / \mathrm{m}^{2}$ and above $25 \mathrm{~kg} / \mathrm{m}^{2}$ morbidity and mortality rates increase. Above $30 \mathrm{~kg} / \mathrm{m}^{2}$ mortality rates from all causes, and especially from cardiovascular disease (CVD), are generally increased by $50-100 \%$ (National Heart Lung and Blood Institute, 1998a). A NHLBI news release (National Heart Lung and Blood Institute, 1998b) stated, 'The evidence is solid that the risk for various cardiovascular and other diseases rises significantly when someone's BMI is over $25 \mathrm{~kg} / \mathrm{m}^{2}$ and that risk of death increases as the BMI reaches and surpasses 30 $\mathrm{kg} / \mathrm{m}^{2}$, The following classifications of overweight and obesity were suggested:

\section{BMI}

Underweight

Normal weight

Overweight

Obesity (Class 1)

Obesity (Class 2)

Extreme obesity (Class 3)

$$
<18.5 \mathrm{~kg} / \mathrm{m}^{2}
$$$$
18.5-24.9 \mathrm{~kg} / \mathrm{m}^{2}
$$$$
25.0-29.9 \mathrm{~kg} / \mathrm{m}^{2}
$$$$
30.0-34.9 \mathrm{~kg} / \mathrm{m}^{2}
$$$$
35.0-39.9 \mathrm{~kg} / \mathrm{m}^{2}
$$

$\geq 40 \mathrm{~kg} / \mathrm{m}^{2}$

Although it is a useful method, the use of BMI as an indicator of overweight can lead to misleading results. For example, an extremely muscular athlete may be diagnosed 
as obese due to the muscle mass giving an increased overall body mass (muscle weighs more than fat) (Heyward \& Stolarczyk, 1996). In response to this problem, for individuals or populations with disproportionate shapes some adjustment can be made. Norgan and Jones (1995) showed that the ratio of sitting height to standing height allows some effective adjustment in populations such as the Australian Aborigines (very tall and lean leading to low level of BMI) or the American Indians (short and stocky leading to a very high BMI). BMI can also be improved in its assessment of obesity by including with it a measurement of waist circumference, which is strongly associated with abdominal fat, an independent predictor of disease risk (National Heart Lung and Blood Institute, 1998b).

The validity of the BMI method is questioned further by studies showing that correlations between percentage body fat (\%BF) and $\mathrm{BMI}$ are not independent of other variables. Wang et al. (1994) demonstrated that the relationship varied by BMI and sex and ethnicity (adults aged 18 - 94 years). Jackson et al. (2002) showed that the relationship was non-linear for both men and women (ages 17-65 years), and that sex and age should be controlled for.

\section{b) BMI in Children}

In a sample of 188 healthy Italian participants aged $5-19$ years, Pietrobelli et al. (1998) found BMI to be strongly associated with \%BF estimated from dual-energy $\mathrm{x}$ ray absorptiometry (DXA) $\left(\mathrm{R}^{2}=.63\right.$ for boys, $\mathrm{R}^{2}=.69$ for girls). However, they also reported wide confidence intervals for the level of association, which suggests that $\mathrm{BMI}$ is a better indicator of $\% \mathrm{BF}$ in groups rather than individuals. Age was a significant covariate for both boys and girls, after controlling for BMI older participants had a lower \%BF, and the authors concluded that interpretation should be cautious when comparing BMI across different age groups.

In fact, if we wish to assess children using BMI, it is necessary to measure them on age-specific scales because their BMI constantly changes as patterns of growth in height and weight change. Between the ages of 1 and 5 years increases in height are greater than increases in weight (BMI decreases), but the trend reverses after 5 years as weight gains become greater than height gains (BMI increases). The pubertal 
growth spurt also serves to complicate measurements which may help explain the lack of association in children $>10$ years of age found by Widhalm et al. (2001). BMI reference curves have been developed using data collected in 1990 that allow the assessment of UK children from birth to 23 years using age-specific BMI centiles (Cole et al., 1995).

Furthermore, Cole, Bellizi, Flegal, and Dietz (2000) linked the adult cut off values of $25 \mathrm{~kg} / \mathrm{m}^{2}$ (overweight) and $30 \mathrm{~kg} / \mathrm{m}^{2}$ (obese) to produce cut off points for BMI in children. This study linked the centiles at age 18 , that passed through the overweight and obese cut offs, to provide age and sex specific cut off points from 2 to 18 years. These cut off points have been associated with estimates of \%BF values in children and adolescents from New Zealand (Taylor, Jones, Williams, \& Goulding, 2002). Using multiple linear regression, Taylor et al. found that children classified as overweight had body fat that ranged from $18-23 \%$ in males and $20-34 \%$ in females. Those classified as obese had body fat values of $24-36 \%$ and $26-46 \%$ (males and females respectively). For US children aged $2-20$ years, growth charts, including an age- and sex-specific BMI reference are also available (Kuczmarski et al., 2000).

Within the current study, it was hypothesised that changes in behaviour associated with body compositional changes during puberty may be partly mediated through body image. For a given pubertal stage and BMI, the effect on behaviour may vary according to body image, which can be affected by the age of the participant. For example, early maturing females may feel different compared to their peers because of an increase in body fat. The relationship between body composition and body image may be explained more easily using BMI centiles in place of BMI because changes in relation to a participant's peers can be more easily seen.

Differences in ethnicity may also affect the use of BMI in children. In a multiethnic sample, aged 5.0 - 10.9 years of age, from New Zealand, Tyrell et al. (2001b) found small but significant $(\mathrm{p}=.0004)$ differences due to ethnicity in the relationship between $\mathrm{BMI}$ and \%BF as predicted using foot-to-foot bioelectrical impedance (BIA); with BMI explaining $60-73 \%$ of the variance in \%BF for European, Maori, and Pacific Island participants. It was noted that this difference may be due to the high 
number of Pacific Island children with BMI $>30 \mathrm{~kg} / \mathrm{m}^{2}$ and that no clinically relevant difference was seen in BMIs $<30 \mathrm{~kg} / \mathrm{m}^{2}$. Although no adjustment for age was made, the age range of this group suggests that participants were less likely to have been affected by the onset of puberty. Use of BIA as the criterion method is itself a source of some error however; in an earlier study by Tyrell et al. (2001a) foot-to-foot BIA was compared with anthropometry and DXA-derived body composition in 85 healthy children of European, New Zealand Maori and Pacific Island origin aged 4.9 - 10.9 years of age. Their results showed that BIA explained $88 \%$ of the variance in $\% \mathrm{BF}$ from DXA, ethnicity was not found to be significant.

Level of body fat may also be a factor in the accuracy of the BMI method. Widhalm, Schönegger, Huemer, and Auterith (2001) demonstrated that, in a large sample of obese children and adolescents, for boys and girls younger than 10 years there was a strong association between $\mathrm{BMI}$ and \%BF measured using total body electrical conductivity (TOBEC) $\left(r^{2}=.73 \& .63\right.$ respectively). However, the association for older children ( $>10$ years), although significant, was considerably less $\left(r^{2}=.27 \& .38\right.$ respectively). They concluded that $\mathrm{BMI}$ is not a reliable measure for the determination of body fat in the individual obese paediatric patient.

Heyward and Stolarczyk (1996) suggested that to assess the body composition of children, the skinfold (SKF) or BIA methods can be used without the inaccuracies described in the BMI method. However, Bellizzi and Dietz (1999) concluded that although BMI is not a perfect measurement of fatness in children, it might be the most appropriate as it has been validated against measurements of obesity and presents less difficulty in measurement than does the skinfold method, although they added that alternative methods of measurement should be considered in the future. Similar arguments were presented by Geiß, Parhofer, and Schwandt (2001) who concluded that, in a group of healthy prepubescent children aged $4-9$ years, BMI was a better predictor of cardiovascular risk factors than obesity parameters using skinfold measurement. In boys, obesity defined by BMI predicted all tested cardiovascular risk factors (HDL-cholesterol, triglycerides, plasma fibrinogen and blood pressure) except LDL-cholesterol and apolipoprotein B. In overweight girls BMI predicted the presence of all CHD risk factors. SKF did not perform as well; in boys SKF failed to predict a significant difference of HDL and LDL-cholesterol, and apolipoprotein B. In 
girls SKF failed to predict a significant difference of HDL and LDL-cholesterol, apolipoprotein B and fibrinogen.

As it was for adult assessments, the addition of a measure of waist circumference into analyses may be advantageous in the estimation of childhood body composition. McCarthy, Ellis, and Cole (2003) found that it was more sensitive to increases in adolescent overweight and obesity compared with BMI. They found that over a 20year period from 1977 to 1997 standard deviation (z) scores for waist circumference in males increased by 0.84 units versus 0.47 units for BMI. These increases represented a $19.8 \%(10.7 \%)$ rise in overweight (obesity) when using waist circumference versus $12.9 \%(6.8 \%)$ when using BMI. The contrast was even greater in females, over a 10-year period from 1987 to 1997 waist circumference z-scores increased by 1.02 units versus 0.53 units for BMI. These increases represented a $29.3 \%(14.5 \%)$ rise in overweight (obesity) using waist circumference versus $11.4 \%$ (6.6\%) when using BMI. The authors suggested that the differences occurred as a result of changing body composition in adolescents; increases in centralised fat mass being obscured by reductions in muscle mass when using BMI. This problem is avoided by use of the waist circumference since it reflects total (visceral and subcutaneous) abdominal fat but not the fat-free mass.

\section{Skinfold Method}

This is a method that uses metal or plastic callipers to measure the thickness of a fold of fat. SKF is a non-invasive and easy to administer method at relatively low cost. Measurements are made at a number of sites on the body; Lohman, Roche, and Martorell (1988) and Cameron (1984) give examples of fourteen. The number and particular sites chosen will depend on the particular method being followed. Because the extent to which the subcutaneous fat reflects total body fat varies among populations and site of measurement, it is important to use procedures that have been standardised for the population being studied. The results are substituted into regression equations that predict \%BF by comparing SKF measurements with results obtained from accepted laboratory methods. 
Hydrodensitometry is one of the most commonly used laboratory methods. This is a 'two-component model' that divides the body into fat and fat-free mass (FFM) components, assuming that the fat and FFM have constant densities $(0.090 \mathrm{~kg} / \mathrm{l}$ for fat; $0.100 \mathrm{~kg} / 1$ for FFM) (Behnke, 1959) with little inter-individual variability (Heyward \& Stolarczyk, 1996). This assumption cannot be made for children because of changes to the proportion and density of FFM during growth and maturation. Therefore, a multi-component approach, which can quantify the FFM composition and measure individual variation, rather than assume it to be constant, is required to develop suitable predictor equations. Multi-components can be measured using isotope dilution (water), dual-energy $\mathrm{x}$-ray absorptiometry (mineral content), and neutron activation analysis (protein) (Heyward \& Stolarczyk, 1996). Multi-component methods allow the development of regression equations that can be used with the specific group being studied, for example, by age (Van Loan \& Mayclin, 1987) or ethnic group (Van Loan \& Mayclin, 1987; Wagner \& Heyward, 2001).

Slaughter et al. (1988) developed the following equations for use in black (AfroAmerican) and white (American) children using triceps and calf, or triceps and subscapular measurements. The study included groups of pre-pubertal, pubertal and post-pubertal children as well as adults.

Striceps and calf

Black and White

Boys (all ages) $\quad \% \mathrm{BF}=0.735(\mathrm{SKF})+1.0 \quad(\mathrm{~A})$

Girls (all ages) $\quad \% \mathrm{BF}=0.610(\Sigma \mathrm{SKF})+5.1$

¿triceps and subscapular; $(\Sigma \mathrm{SKF}>35 \mathrm{~mm})$

Black and White

Boys (all ages) $\quad \% \mathrm{BF}=0.783(\mathrm{SSKF})+1.6 \quad(\mathrm{C})$

Girls (all ages) $\quad \% \mathrm{BF}=0.546(\Sigma \mathrm{SKF})+9.7 \quad(\mathrm{D})$

Striceps and subscapular; $(\Sigma \mathrm{SKF}<35 \mathrm{~mm})$

Black and White

Boys (all ages) $\quad \% \mathrm{BF}=1.21(\Sigma \mathrm{SKF})-0.008(\Sigma \mathrm{SKF})^{2}+\mathrm{I}^{*}$ 
*I = intercept substitutions based on maturation and ethnicity for boys:

$\begin{array}{lll}\text { Maturation } & \text { Black } & \text { White } \\ \text { Pre-pubertal } & -3.2 & -1.7 \\ \text { Pubertal } & -5.2 & -3.4 \\ \text { Post-pubertal } & -6.8 & -5.5 \\ \text { The standard errors of estimate (SEE) range from 3.2\% to } 3.9 \% \text {. }\end{array}$

The ages of the participants in this study were as follows:

\begin{tabular}{llll} 
& N & Mean Age & SD \\
\hline Prepubertal & 42 & 9.9 & 1.1 \\
Pubertal & 23 & 12.5 & 1.2 \\
Post-pubertal & 60 & 15.5 & 1.3 \\
Adult & 48 & 22.6 & 2.7
\end{tabular}

It may be difficult to measure the skinfolds of particularly obese or heavily muscled individuals as it may not be possible to pick up a fold with parallel sides and the callipers have a limit as to how wide they will open (Gray et al., 1990). Cameron (1984) stated that skinfolds may be difficult to obtain in the more obese participant with skinfolds greater than 20 to $25 \mathrm{~mm}$ - above the $97^{\text {th }}$ centile of British charts. Conversely, with very lean individuals it may be difficult to separate the adipose layer from the underlying muscle (Lohman et al., 1988).

Errors can be produced from the accuracy of the original reference model, Lohman (1981) estimated the error in predicting body fat from densitometry to be $\sim 2.5 \%$, but also by variability between researchers in taking measurements. A great deal of practise is required to achieve an acceptably high level of skill. Jackson and Pollock (1985) and Katch and Katch (1980) (both cited in Heyward and Stolarczyk, (1996)) recommended practising the skinfold technique on $50-100$ participants in order to develop the required skill level. 
Greater errors can occur from how the researcher collects the fold of tissue rather than where (Cameron, 1984). Lohman (1992) listed examples of technical sources of error as: skinfold measurement technique, skinfold site location, and skinfold compressibility. The choice of callipers can also affect the measurements. Using the same callipers consistently helps to reduce equipment error. Project STIL used Holtain callipers, an improved version of the Harpenden callipers described as 'robust and reliable' (Cameron, 1984). Using standardised procedures, such as those given in Cameron (1984) or Lohman et al. (1988), also helps to reduce error as these were developed to minimize sources of variation between researchers.

\section{Bioelectrical Impedance Method}

This is a rapid, cheap, non-invasive, and painless (Schaefer, Georgi, Zieger, \& Scharer, 1994) method that requires a less high level of technical skill and is less invasive to the participant than the skinfold method (Lukaski, Johnson, Bolonchuk, \& Lykken, 1985; Segal, Van Loan, Fitzgerald, Hodgdon, \& Van Itallie, 1988; Van Loan \& Mayclin, 1987). BIA estimates free-fat mass (FFM) by passing a low-level electrical current through a participant's body and measuring the impedance $(\mathrm{Z})$.

Impedance is a measure of the opposition to the flow of current and can be expressed as:

$$
Z=\sqrt{ }\left(R^{2}+X_{c}^{2}\right)
$$

Where $\mathrm{R}$ is resistance and $\mathrm{X}_{\mathrm{c}}$ is reactance.

A $50 \mathrm{kHz}$ current at $800 \mu \mathrm{A}$ is used as at this frequency the current passes through extracellular fluids and not intracellular fluids (Lukaski et al., 1985). This means that at $50 \mathrm{kHz}$ the impedance reflects only the volumes of water and muscle that make up the FFM and extracellular water volume since fat is mainly anhydrous and therefore a poor conductor.

The most common form of use is the tetrapolar method. Here, two pairs of electrodes are placed onto the participant. The current electrodes are placed on the dorsal surfaces of the right hand and right ankle at the distal metacarpals and metatarsals respectively. The detector electrodes measure the voltage drop caused by impedance 
by being placed $5-20 \mathrm{~cm}$ proximal to each current electrode (Baumgartner, Ross, \& Heymsfield, 1998). The participant is required to lie supine, all skin contact areas must be cleaned with alcohol and a thin layer of electrolyte gel applied to the electrodes (Lukaski et al., 1985), thus, this method can be time consuming with a large number of participants.

The BIA method is based on the assumptions that the body is a perfect cylinder and that a constant $50 \mathrm{kHz}$ the following relationship is true:

$$
\mathrm{Z}=\rho \text { (HT/A) }
$$

Where $\rho=$ specific resistivity of the body (constant); HT = body height; $\mathrm{A}=$ body's cross-sectional area.

Further, by multiplying by HT/HT the equation becomes:

$$
\begin{aligned}
& Z=\rho(\mathrm{HT} / \mathrm{A})(\mathrm{HT} / \mathrm{HT}) \\
& Z=\rho\left(\mathrm{HT}^{2} /[\mathrm{A} \times \mathrm{HT}]\right)
\end{aligned}
$$

Since Ax HT is the volume of the FFM $\left(\mathrm{FFM}_{\mathrm{v}}\right)$, then:

$$
\mathrm{Z}=\rho\left(\mathrm{HT}^{2} / \mathrm{FFM}_{\mathrm{v}}\right)
$$

Rearranging gives:

$$
\mathrm{FFM}_{\mathrm{v}}=\rho\left(\mathrm{HT}^{2} / \mathrm{Z}\right)
$$

Using equation (1), at $50 \mathrm{kHz} R$ is much greater than $\mathrm{X}_{\mathfrak{c}}$, therefore:

$$
\sqrt{ }\left(\mathrm{R}^{2}+\mathrm{X}_{\mathrm{c}}{ }^{2}\right) \approx \mathrm{R}
$$

Equation (6) can then be expressed as:

$$
\mathrm{FFM}_{\mathrm{v}}=\rho\left(\mathrm{HT}^{2} / \mathrm{R}\right)
$$

$\mathrm{HT}^{2} / \mathrm{R}$ is normally expressed as the resistance index RI. 
In order to convert a volume reading into a mass reading assumptions must be made regarding the density of the tissues that make up the human body. As for the SKF method a multi-component method must be used to develop equations for children and adolescents.

The following equations are suitable for children and adolescents:

Boys and girls (10-19 yr)

$\mathrm{FFM}(\mathrm{kg})=0.61 \mathrm{RI}+0.25 \mathrm{BW}+1.31$

(G) Houtkooper (1992)

Boys and girls (8-15 yr)

$\operatorname{FFM}(\mathrm{kg})=0.62 \mathrm{RI}+0.21 \mathrm{BW}+0.10 \mathrm{X}_{\mathrm{c}}+4.2 \quad(\mathrm{H})$ Lohman $(1992)$

$\mathrm{BW}=$ body weight $(\mathrm{kg})$

$\%$ Body fat is then calculated by:

Fat Mass $(\mathrm{FM})=$ Body Mass $(\mathrm{BW})-\mathrm{FFM}$

$\% \mathrm{BF}=\mathrm{FM} \div \mathrm{BW} \times 100$

A number of studies have been undertaken to test the validity of the BIA method in predicting FFM in children. Houtkooper et al. (1989) found that RI together with anthropometry was a reliable and acceptably accurate method of predicting children's FFM and \%BF obtained from measures of body density, weight and water, and using equations developed from multi-component methods. Including measurements of chest circumference and hip skeletal width along with $\mathrm{RI}$ in the prediction equation resulted in a standard error of estimate (SEE) of 2.0kg FFM. A follow up crossvalidation study by Houtkooper et al. (1992) produced equation (G) that used RI + weight to predict FFM with moderately small errors. The SEE was $2.1 \mathrm{~kg}$ which represents $4.5 \%$ error in a $55 \mathrm{~kg}$ child with $15 \% \mathrm{BF}$. The SEE for Lohman's equation was also $2.1 \mathrm{~kg}$. 
These equations are limited to healthy white children using the same equipment used in each study (model 101, RJL Systems, equation (G) and Valhalla Scientific equation (H)). It was also noted by the authors that the effects of ethnicity, alteration in hydration, pre-measurement exercise and eating could affect results because these were held constant when producing the prediction equations $(\mathrm{G})$ and $(\mathrm{H})$.

Schaefer et al. (1994) found that for children aged 3.9 - 19.3 years BIA was comparable to SKF methods when predicting FFM derived from total body potassium. Their measurements produced $\mathrm{R}^{2}$ values of $0.964-0.975$ for BIA compared with $0.973-0.975$ for SKF. They also found that intraobserver and interobserver reproducibility was far better with BIA although overall technical errors were similar for both methods.

BIA can be used more easily to measure obese individuals although may slightly underestimate the \%BF (Baumgartner et al., 1998; Gray, Bray, Gemayel, \& Kaplan, 1989; Segal, Gutin, Presta, Wang, \& Van Itallie, 1985; Segal et al., 1988). Gray et al., cross-validating the results of Segal et al. (1988), suggested that caution should be used when measuring very obese participants with body fat of greater than $48 \%$. In these participants the FFM derived from impedance equations was overestimated ( $5 \mathrm{~kg}$ greater than FFM determined from body density measured by underwater weighing). This may be due to a change in the body composition of very obese participants, such as expanded extracellular water or increased intramuscular fat. These changes would affect the volume resistivity of the tissues and therefore bias any calculations made using impedance.

An alternative method of placement is the 'foot-to-foot' method described by Nuñez et al. (1997) and validated for use with children by Tyrell et al. (2001a). This is a more convenient method than that described by Lukaski et al. (1985) whereby the participant stands on four electrodes in the form of stainless steel foot-pads mounted on the top surface of a platform scale. This allows simultaneous measurement of the participants' leg-to-leg impedance and body weight. Other variables such as gender can be entered into the LCD screen on top of the machine. Measurements can be done in one minute (Tyrrell et al., 2001a), an advantage for large epidemiological studies where much time can be spent on measurements. 
The measurement of impedance from the extremities only has previously been found to give a reasonable prediction of arm-to-leg impedance and body composition (Baumgartner, Chumlea, \& Roche, 1989). $\mathrm{R}^{2}$ values ranged from $0.61-0.75$ for whole body impedance and $0.42-0.65$ for impedance using arms or legs only when compared with \%BF from densitometry. This suggests that the tetrapolar method can be replaced by a method that measures only the impedance of extremities such as the legs. Nuñez et al. (1997) found that correlation coefficients for leg-to-leg and arm-toleg BIA systems, when compared with body composition components, had similar correlation coefficients and SEEs, especially when age, gender and waist/hip circumference ratio were added to RI in the regression models. They concluded that, for non-obese adults, this new BIA system has overall performance characteristics for impedance measurement and body composition analysis similar to conventional armto-leg methods with the added advantage of ease and speed of use.

In the study by Tyrell et al. (2001a) foot-to-foot BIA was compared with anthropometry and DXA-derived body composition in 85 healthy children of European, New Zealand Maori and Pacific Island origin aged 4.9-10.9 years of age. Their results showed that BIA correlated better with DXA than did anthropometrical measurements in the estimation of fat-free mass, fat mass, and $\%$ body fat $(r=.94-$ .98 versus $\mathrm{r}=.41-.94$ respectively).

The prediction equation derived from their results was:

FFM $(\mathrm{kg})=0.31 \mathrm{RI}+0.17 \mathrm{HT}+0.11 \mathrm{BW}+0.942 \mathrm{sex}-14.96$

(where sex $=1$ for females and 2 for males)

Pubertal stage, age and ethnicity were not included, as they were not found to be significant factors.

When using monitors from the Tanita Company (Tanita UK Ltd, Middlesex, UK) it is possible to receive a direct readout of $\% \mathrm{BF}$ from the screen on top of the scales as the device calculates them using built-in equations. For the most accurate readout gender and height must be entered before use, body weight is calculated as the participant is on the scales. 
Fat percentages of 40 prepubertal children ( 22 girls, mean age 7.0 years; 18 boys, mean age 7.0 years) and fifty of their parents (27 mothers, mean (SD) age 36.3 (5.2) years; 23 fathers, mean (SD) age 39.3 (5.2) years), based on the direct readouts of a Tanita 105, were taken by Jartti et al. (2000) and correlated with \%BF calculated from a hand-to-leg BIA device. BIA measurements were taken after an overnight fast to prevent the decrease in impedance (and underestimation of body fat mass) that can occur 2-5 hours after breakfast (Gallagher, Walker, \& O'Dea, 1998). BMI and waistto-hip ratios were also calculated.

With the hand-to-leg analyser body fat was calculated using the Houtkooper's equation (G) and also the inbuilt equation of the device, which gave "almost identical values" (Jartti et al., 2000, p.782). The Tanita 105 provided output values for weight, impedance, fat-free mass, total body water, and fat percentage. The output value for fat percentage was used in the final analysis.

Correlations between the impedances obtained from each device were high over the whole range of impedance values $(r=.95, r=.93, r=.82$, and $r=.81$ for girls, boys, mothers, and fathers respectively; $p<.0001$ ). However, when comparing the fat percentages obtained by the two devices correlations were high in girls, mothers, and fathers $(r=.71, .94, .80$ respectively; $p<.001)$ but low in boys $(r=.21, p=.41)$. Therefore \%BF readings straight from the Tanita 105 scales should be used with caution.

\section{Summary of Body Composition Assessment}

All methods of body composition measurements are subject to varying degrees of measurement error (Segal et al., 1985). For example, because Lohman (1981) estimated the error of the densitometric method for measuring $\% \mathrm{BF}$ as $\sim 2.5 \%$, then, if this was the reference model for other methods, such as SKF, this would be the maximum possible accuracy.

BMI is a popular and commonly used method for measuring obesity in adults but is affected by body compositional changes during puberty and may also be unsuitable 
for use with obese children. However, a workshop organised by the International Obesity Task Force (Bellizzi \& Dietz, 1999) recommended that although BMI is not a perfect measure of obesity in children, it should be used, as it is an easy measure of body fat that is reproducible and valid. They added that a clinical decision about whether a child with a given BMI is truly overfat may require additional information including SKF measurements. The use of BMI centiles may be a more appropriate method of explaining behavioural changes since they take into account changes in relation to a participant's peers that may have an effect on body image. The addition of waist circumference measures, for example the waist-hip ratio, may also help to improve the assessment of body compositional changes.

For the SKF method, other sources of error include the accuracy of the prediction equation in estimating \%BF when compared to the reference model and the variability of the researchers taking the measurements. Systematic errors can be reduced by using procedures and equipment identical to those of the original investigation. SEEs for equations (A) - (F) range from 3.2\% (pubescent participants $\Sigma$ triceps and subscapular), to 3.9\% (Etriceps and calf) (Slaughter et al., 1988). An additional source of error may be the use of inappropriate prediction equations for the population under investigation, equations (A) - (F) were designed for black and white participants within the age range of this study, thus keeping this type of error to a minimum.

SEEs for BIA equations $(\mathrm{G})$ and $(\mathrm{H})$ were $2.1 \mathrm{~kg}$ (Houtkooper et al., 1992; Lohman, 1992) (approximately $4.5 \%$ in a $55 \mathrm{~kg}$ participant with $15 \% \mathrm{BF}$ ). These equations were designed for white children $10-19$ years and $8-15$ years, however the recent addition of a foot-to-foot BIA system that has been tested on a multi-ethnic group of prepubescent and pubescent participants has provided an alternative that is quick and easy to administer with results that are comparable to other BIA methods.

\section{Assessment of Physical Self-Perception}

The Physical Self-Perception Profile (PSPP), developed by Fox and Corbin (1989), was adapted for use with younger people by Whitehead (1995) and renamed the 
Physical Self-Perception Profile for Children (C-PSPP). In a study involving 505 seventh- and eighth-grade students (age range approximately $11-15$ years - not reported) a questionnaire was administered which contained a global self-worth scale (GSW), a physical self-worth scale (PSW), and four subscales representing Fox and Corbin's four sub-domains of PSW from the PSPP (Sports Competence, Physical Condition, Body Attractiveness, and Physical Strength). The results from this study supported the reliability and the construct and concurrent validity of these scales for this age group.

The second part of Whitehead's (1995) study aimed to assess participants' perceptions of the importance of being competent or adequate in each of the C-PSPP subdomains. This Children's Perceived Importance Profile (C-PIP) is also an adapted version of a scale developed by Fox and Corbin (1989), which includes two questions regarding the importance given by the participant to each of the four subdomains. These questions also follow the structured alternate format (Harter, 1982). Even though the results from the second part of this study failed to demonstrate factorial validity for the C-PIP, the relationship between perceptions in the sub-domains and global perceptions that was observed supported the discounting hypothesis discussed earlier. Whitehead concluded that further work is needed on these scales to identify whether a factor structure failed to emerge because perceived importance perceptions are relatively undifferentiated within this age group or because the items within the scale were poorly written.

\section{Assessment of Maturation}

In order to assess changes in sedentary behaviour that are associated with puberty and adolescence it is necessary for studies to include a measure of pubertal stage in addition to age. Within populations of children and adolescents, measurement of pubertal status must be carried out in a non-intrusive way that does not require the undressing of the participant and does not cause any undue embarrassment.

One option for the researcher is the viewing of photographs that show different stages of secondary sexual characteristic development. For females there are photographs 
showing representations of the five stages of breast development (B1, B2, B3, B4, B5) and five stages of pubic hair growth (PH1, $\mathrm{PH} 2, \mathrm{PH} 3, \mathrm{PH} 4, \mathrm{PH} 5)$. In males there are five photographs showing the stages of genital development (G1, G2, G3, G4, G5) and five showing pubic hair development (Tanner, 1962). For boys the two sets of photographs can be combined together (Duke, Litt, \& Gross, 1980). All of the photographs include a short descriptive paragraph. Participants are required to pick the photograph (two for females and one or two for males) that most accurately depicts their own stage of development.

The use of this procedure as a self-evaluation method has been validated by a number of studies. Duke et al. (1980) compared the self-assessed results from 43 female adolescents aged $9-17$ years ( 5 black, 38 white), and 23 male adolescents aged 11 18 years ( 3 black, 20 white) to those taken during a physical examination by the investigators. Agreement with the investigator rating occurred for breast stage in $37 / 43$ (kappa coefficient $\kappa=.81$ ), for female pubic hair stage in $40 / 43(\kappa=.91)$, and for male combined pubic hair and genital stage in $21 / 23(\kappa=.88)$. Only one boy differed from the physicians by more than one stage. The authors concluded that, "In future research projects, investigators can allow teenagers to accurately designate their own level of sexual maturation" (p. 919).

The results of Duke et al. (1980) may be compared to those of Neinstein (1982) who administered the test to a mixed ethnic group of adolescents of a lower socioeconomic status than those of the previous study. Forty-four participants of ages 11 18 years (22 female and 22 male) recorded their own rating and again these were compared to a physician rating from a physical examination. The results showed agreement in 17/22 $(r=.87)$ breast stage assessments, $16 / 22(r=.86)$ female pubic hair assessments, $10 / 22(r=.73)$ male genital assessments, and 11/22 $(r=.69)$ male pubic hair assessments. The absolute differences in scoring were also calculated and were found to be 0.28 (breast rating), 0.48 (pubic hair development), and 0.68 (genital rating). Only four adolescents differed from the physicians by more than one stage (three male, one female). No significant differences for sexual maturity rating were found between groups based on race, however when total differences between adolescent and physician rating were compared between males and females a significant difference was found (male $=1.32$, female $=0.60, p<.05$ ). Neinstein 
suggested that the differences in findings between this study and that of Duke et al. may be due to differing socio-economic status, a different recruitment method, or the presence of more mature participants. In fact, further analysis showed a greater scoring disparity with increasing age for males $(r=.53, p<.05)$ but not for females $(r$ $=.04, p>.05)$. It was concluded that this method of self-assessment is feasible for black, white, and Hispanic females, but further research was needed before using the method with adolescent males.

Use of this method with younger female adolescents has been validated by Frankowski et al. (1987b). Results with 66 girls (aged 7 - 12 years) compared favourably with those of the previously described studies with agreement in 40/66 $(r=$ $.75, \mathrm{p}<.001, \mathrm{k}=.56)$ for breast stage, and 52/66 $(\mathrm{r}=.88, \mathrm{p}<.001, \mathrm{k}=.70)$ for pubic hair. In contrast, the results from 32 boys (aged $9-12$ years) showed agreement in only $15 / 32(r=.25, p<.01, k=.20)$ for genital development and 14/32 $(r=.44, p<$ $.01, \kappa=.15$ ) for pubic hair development; many of the pre-pubertal boys overestimated their developmental stage. Thus, the method is suitable for girls in the age group 7 12 years but less so for boys aged $9-12$. Although these students were generally younger than those involved with Project STIL the results suggested that the method must be used with caution when assessing boys from the lower stages of maturation.

Lower concordance between physicians' ratings and those of boys compared with girls was also found in a study with boys and girls in Brazil (Matsudo \& Matsudo, 1994). Using a similar protocol to those in the studies above, the authors reported overall concordance of $60 \%$ (boys) to $70 \%$ (girls), ranging from between $23.0 \%$ (breast stage 4) to $100.0 \%$ (breast stage 5). Concordance was greater for stages 1 and 5 with greater variation in the intermediate stages. The magnitude of error was relatively small however with most differing assessments no more than one stage apart. Reproducibility of the method was also assessed and found to be good with Spearman correlations ranging from $r=.76$ to .99 . It was concluded that despite the limitations of the self-assessment method it did have the potential for use given its simplicity, applicability, economy, reasonable reproducibility, and respect for individual privacy. 
Alternative methods for non-invasive evaluation of maturity are available. For example, Beunen et al. (1997) used the percentage of adult stature achieved as an indicator of biological maturation because a higher percentage of adult height reached represents a more advanced maturity status. It was suggested that this method is advantageous over the use of secondary sexual characteristics because the latter method often has cultural limitations. The authors used this method to classify 14year-old Belgian boys into early-, average-, and late-maturers. The advantage, for the current study, of the former method (Tanner, 1962) compared with that of Beunen et al. is that patterns of inactivity may be affected by the somatic changes of puberty rather than simply maturational status alone and the latter method does not include pubertal landmarks.

Brooks-Gunn et al. (1987) suggested alternative methods of assessment that could be used when approval for self-assessment by Tanner stages is not provided, particularly in school based studies. In this paper the accuracy of three methods was assessed by comparison with physician ratings of Tanner stages (assumed to be the 'gold standard'). The three methods assessed were:

1. Self-assessment using schematic drawings of Tanner stages as described above.

2. Parental assessment using the same drawings.

3. Self-assessment using the Pubertal Development Scale (PDS).

The PDS is a 4-point scale that rates development of four somatic characteristics (growth spurt in height, body hair, skin change, and breast development). This method uses an interview or questionnaire format rather than drawings or photographs and is designed to be more acceptable for participants to whom looking at naked diagrams may be unacceptable.

The participants in this study were 151 white girls, $11-13$ years of age and from welleducated, middle- to upper-middle-class families. Each girl undertook a physician rating of maturity and was also rated using each of the three methods outlined above. The results from the self-assessments compared well with the studies previously described, correlations across all the age groups between physician and girl were $r=$ 
.72 (breast growth), .80 (pubic hair growth), and .82 (a combined rating). The authors noted that the girls were not given the chance to see themselves in the mirror whilst they were assessing themselves, if they had been then correlations may have been higher and future studies should include this opportunity.

In addition, correlations between physician and mother were also high as follows: $r=$ .80 (breast growth), .79 (pubic hair growth), and .85 (combined rating). All correlations using the Tanner stage schematics were significant at $\mathrm{p}<.001$. Correlations between the PDS score and physician rating were also significant at the same level but lower overall: $r=.61$ (breast growth), .62 (pubic hair growth), and .67 (combined rating). It was concluded that all three methods are an acceptable alternative to physician rating in situations such as school-based studies. The suggested order of preference was:

1. Self-assessment using schematic drawings of Tanner stages.

2. Parental assessment using the same drawings if school staff or local school boards do not feel that young adolescents should be asked to view photographs or diagrams of sexually developing girls.

3. Self-assessment using the Pubertal Development Scale if either parental or adolescent ratings of drawings are unacceptable.

Because this study was involved with girls only, the authors were unable to recommend use of any of the methods with male adolescents. They pointed out that in particular assessment by mothers might not be possible with boys.

It should be noted at this point that use of the correlation coefficient or percentage agreement to support conclusions regarding agreement between different raters has been brought into question. To quote Howitt and Cramer (2000), “...correlation coefficients are not very helpful when the exact agreement of raters is required" ( $p$. 404). A more appropriate statistic is the kappa coefficient ( $\mathrm{k}$ ) (because it is sensitive to the need for raters to agree at all levels of any scale), which was not included in all of the preceding studies. 
Another alternative, non-invasive method of assessment is to use particular landmarks of puberty to grade a participant as pre-pubertal, early-pubertal, late-pubertal, or adult. Studies have shown variations in the timing and duration of pubertal events although general patterns of development have been described (Marshall \& Tanner, 1969, 1970), for example in both males and females the beginning of pubertal hair growth (PH2) will indicate that puberty has started and can therefore distinguish between those who are pre-pubertal and those who are pubertal/adult.

In females another commonly used landmark is menarche, an event that occurs approximately two years after the onset of puberty, during B4, and up to a further two years before maturity is reached (Tanner, Whitehouse, Marubini, \& Resele, 1976), thus distinguishing between early- and late-puberty. Unfortunately no such obvious event exists in the development of males. However, one landmark that could be useful is the point at which the voice breaks. This measure was included in a study by Billewicz, Fellowes, and Thompson (1981) in which 669 boys and 753 girls were observed at approximately 6 monthly intervals from 9 to 17 years of age and mean ages of reaching various stages of development were calculated. In the boys, sexual maturation was estimated by measuring axillary hair, stage of voice breaking, and amounts of facial hair. Voice breaking was found to start at around 14.6 years and be completed by 15.5 years, ages that approximate to G4 - G5 and PH4 - PH5 according to Marshall and Tanner (1970). This implies that voice breaking occurs in mid- to late-puberty similar in timing to that of menarche in females, the onset being up to 2 years before puberty is completed. Therefore, by assessing if and when the onset of menarche/voice breaking occurred we are able to classify non-pre-pubertal students as early- or late-pubertal or adult.

Tanner (1989) however, suggested that voice breaking is not reliable as a criterion of puberty. An alternative measure therefore, may be the appearance of axillary hair, which, according to Tanner, appears usually only when pubic hair has reached stage 4. In the aforementioned study of Billewicz et al. (1981), axillary hair was found to appear at around 14.32 years for boys and be completed by 15.64 years. These ages also approximate to G4 - G5 and PH4 - PH5 according to Marshall and Tanner (1970). 
Another alternative activity that may be used to classify males is the onset of shaving. If it can be shown that facial hair that is long enough to be shaved is associated with a particular stage of puberty then this landmark could also be used. Facial hair growth is determined by testosterone (T) and its metabolite 5a-dihydrotestosterone (DHT). Research findings have suggested that $\mathrm{T}$ is responsible for hair follicle priming (control of hair density) whereas DHT promotes linear hair growth (Farthing, Mattei, Edwards, \& Dawson, 1982). Other researchers have measured the changes in plasma levels of DHT throughout puberty and their results can be summarised as shown in Table 3.6.

A particularly notable aspect of these studies (Ducharme et al., 1976; Gupta, Attanasio, \& Raaf, 1975; Korth-Schutz, Levine, \& New, 1976) is the low participant numbers at each stage that may render the results questionable. Although the results of Gupta et al. (1975) did not list the numbers at each stage, because it was a study of cross-sectional design and there were only 44 boys and 43 girls in total then it is clear that this study also suffered from low numbers. However, the approximate pattern that emerges from each study in Table 3.6 is of similar levels, and increases in DHT in both boys and girls before Tanner stage 3 (boys assessed according to genital size and girls according to breast size), followed by a significant increase in the boys' levels after this stage and a plateauing in the levels of the girls. This may explain the appearance in facial hair in males but not females and suggests that this appearance would be noticeable after Tanner 3 .

Another study that could be used to support this notion is that of Billewicz et al. (1981), in which the grades of facial hair were as follows:

- FH1 - none: down only

- FH2 - Increase in length, pigmentation of hair at corners of upper lip, spreading medially to complete moustache

- FH3 - Hair on upper part of cheeks and in the mid-line just below the lower lip

- FH4 - Hair on sides and lower border of chin 
The mean $( \pm S D)$ age at reaching FH2 $(n=141)$ was $14.67( \pm 1.33)$, which supported earlier results from Neyzi, Alp, Yakacikli, and Orphon (1975) who found that in 1530 schoolboys from Istanbul, Turkey, the mean age of onset of facial hair (FH2) was 14.72 years. This data corresponds to approximately G3 - G4 from the data of Ducharme et al. (1976) given in Table 3.6 and to PH4 - PH5 from the data of Marshall and Tanner (1970). Taken altogether, this evidence suggests that the appearance of facial hair would place boys in the mid-to-late pubertal stage, which corresponds reasonably to the level suggested by the increase in DHT.

Longitudinal data from Dubas, Graber, and Petersen (1991) suggested that there may exist a difference between adolescents' perception of pubertal timing and an objective timing measure. The authors stated that, "It remains to be seen whether one's perception of pubertal timing is a more potent factor than one's actual pubertal timing for influencing other aspects of behaviour" (p. 585). In addition, Dorn, Susman, and Ponirakis (2003) warned against drawing conclusions about pubertal timing and adolescent behaviour when rater of pubertal development varies across studies. Utilising the results of 52 girls, age $9-14$ years, and 56 boys, age $10-15$ years, they found more significant findings between pubertal timing and self-image when rating was by physical examination rather than self-report or parent-report. They concluded that, "It may be important...for investigators to follow theoretical perspectives to determine what measure (or measures) of pubertal timing is relevant to the outcome under study..." (p. 165). Part of the hypothesis of the current study is that earlier maturing participants may change their BMI centile in relation to their peers, which may have a behavioural effect. This behavioural effect is based on a link between body composition and self-perceived body image; therefore it is appropriate to use the participants' self-reported pubertal landmarks in order to assess pubertal stage. 
Table 3.6. Comparison of results from three studies measuring serum/plasma levels of DHT during puberty.

\begin{tabular}{rcccc}
\hline$\delta$ Serum conc. $\mathrm{ng} / \mathrm{dl}(\mathrm{n})$ & $\sim 4(4)$ & $\sim 13(2)$ & $\sim 29(3)$ & $\sim 33(4)$ \\
† Serum conc. $\mathrm{ng} / \mathrm{dl}(\mathrm{n})$ & $\sim 4(13)$ & $\sim 8(12)$ & $\sim 12(7)$ & $\sim 17(6)$ \\
Tanner Stage & 1 & 2 & 3 & $4 / 5$
\end{tabular}

DHT was higher in males in stages 3 and $4 / 5(p<.01)$.

In both sexes differences between stages 1 and 2 were significant $(p<.001)$ (Korth-Schutz et al., 1976).

\begin{tabular}{rllllll}
\hline o Plasma conc. ng/dl (n) & $2.0(1)$ & $3.4(3)$ & $2.6(7)$ & $3.9(10)$ & $13.4(11)$ & $31.8(7)$ \\
Tanner Stage & $1.0 \pm 0.0$ & $1.0 \pm 0.0$ & $1.0 \pm 0.3$ & $1.5 \pm 0.5$ & $2.9 \pm 0.8$ & $4.6 \pm 0.5$ \\
& & & & & & \\
O Plasma conc. ng/dl (n) & $1.4(4)$ & $2.8(4)$ & $6.1(8)$ & $7.7(7)$ & $11.7(7)$ & $7.1(5)$ \\
Tanner Stage & $1.0 \pm 0.0$ & $1.0 \pm 0.0$ & $1.1 \pm 0.2$ & $2.3 \pm 0.7$ & $3.1 \pm 1.1$ & $4.2 \pm 0.4$ \\
Age (years) & $<6$ & $6-8$ & $8-10$ & $10-12$ & $12-14$ & $>14$
\end{tabular}

Significant increases were seen in males only after $10-12$ years and $12-14$ years (Ducharme et al., 1976).

\begin{tabular}{rllllll}
\hline T Plasma conc. ng/dl & $\sim 7$ & $\sim 16$ & $\sim 21$ & $\sim 33$ & $\sim 42$ & $\sim 58$ \\
† Plasma conc. ng/dl & $\sim 5$ & $\sim 9$ & $\sim 16$ & $\sim 17$ & $\sim 17$ & $\sim 17$ \\
Tanner Stage & $1-$ & $2-$ & $3-$ & $4-$ & $5-$ & adult
\end{tabular}

Significant increments were seen in males between stages $3 \& 4,4 \& 5$, and $5 \&$ adult (p<.01) (Gupta et al., 1975).

\section{Summary of Maturation Assessment}

Comparison between the five studies that validated the use of Tanner photographs described can be seen in Table 3.7. These results show that in all five studies the results for females were generally acceptable. The results for males were also acceptable in the studies of Duke et al. (1980) and Matsudo and Matsudo (1994) but were too low in the studies by Neinstein (1982) and Frankowski et al. (1987a). In these latter studies there were a greater number of participants at the extremes of maturational status and this could be the reason for the lower levels of agreement although Matsudo and Matsudo reported greater variation within the intermediate stages and more agreement at the extremes. Care should be taken however when using 
correlation coefficients to compare grades between raters, the kappa coefficient being more appropriate where reported.

The general consensus of these authors is that the method can be used as an estimating tool due to its convenience but that results for females will be more accurate than those for males. Alternative methods for females are mothers' classification using the Tanner stages or use of the PDS. The method of Beunen et al. (1997) measures maturational status but not pubertal status and therefore is not appropriate for Project STIL because patterns of inactivity may be related to somatic changes due to puberty and not maturity alone.

The use of self-assessed pubertal landmarks such as pubic hair growth, menarche for girls, and voice breaking or axillary hair growth for boys are particularly useful in a school setting because of their non-invasive methodology. As with using Tanner photographs, this method will be more accurate for females than for males because the start of menarche is much easier to pinpoint that the onset of voice breaking or axillary hair growth, however, it does allow the classification of participants as prepubertal, early-pubertal, late-pubertal or adult. The use of facial hair growth in boys could be used to grade participants as mid-to-late pubertal if facial hair has appeared, however it does not allow differentiation between pre- and early-puberty.

Table 3.7. Percentage agreements between self- and physician assessments of sexual maturation in a variety of studies.

\begin{tabular}{rlllc}
\hline & $\begin{array}{l}\text { Breasts } \\
\%(\mathrm{r})[\mathrm{k}]\end{array}$ & $\begin{array}{l}\text { O Pubic Hair } \\
\%(\mathrm{r})[\mathrm{k}]\end{array}$ & $\begin{array}{l}\text { Genitalia } \\
\%(\mathrm{r})[\mathrm{k}]\end{array}$ & $\begin{array}{c}\delta^{\mathrm{A}} \text { Pubic Hair } \\
\%(\mathrm{r})[\mathrm{k}]\end{array}$ \\
\hline Duke et al. $(1980)$ & $86 \%(-)[.81]^{\mathrm{d}}$ & $93 \%(-)[.91]^{\mathrm{d}}$ & \multicolumn{2}{c}{$91 \%(-)[.88]^{\mathrm{d}}$} \\
Neinstein $(1982)$ & $77 \%(.87)^{\mathrm{d}}$ & $73 \%(.86)^{\mathrm{d}}$ & $45 \%(.72)^{\mathrm{o}}$ & $50 \%(.69)^{\mathrm{o}}$ \\
Frankowski et al. $(1987)$ & $61 \%(.75)^{\mathrm{a}}[.56]$ & $79 \%(.88)^{\mathrm{a}}[.70]$ & $47 \%(.25)^{\mathrm{b}}[.20]$ & $44 \%(.44)^{\mathrm{b}}[.15]$ \\
Matsudo \& Matsudo (1994) & $61 \%(.92)^{\mathrm{a}}$ & $71 \%(.92)^{\mathrm{a}}$ & $60 \%(.80)^{\mathrm{a}}$ & $70 \%(.84)^{\mathrm{a}}$ \\
Brooks-Gunn et al. (1987) & $(.72)^{\mathrm{a}}$ & $(.80)^{\mathrm{a}}$ & -- & -- \\
\hline
\end{tabular}

${ }^{\mathrm{a}} \mathrm{p}<.001 ;{ }^{\mathrm{b}} \mathrm{p}<.01 ;{ }^{\circ} \mathrm{p}<.05 ;{ }^{\mathrm{d}}$ significance not given. 


\section{Chapter 4: Methods}

\section{Free-Time Behaviour}

Students who took part for the complete duration of the study were assessed on three occasions approximately 6 months apart. Phase 1 assessments took place in spring (March - June) 2002, Phase 2 took place in autumn (October - December) 2002, and Phase 3 took place in spring (March - May) 2003. In this way it was hoped that seasonal variations could be accounted for whilst retaining fairly benign times of the year (in terms of temperature and weather) that would not affect behaviour to too great an extent.

Participants kept the 15-minute MTS diary for three weekdays, which were aggregated into one for analysis, and one weekend day. The actual recording days and the order in which they were completed was randomised among individuals. During the anthropometric assessment the diary procedure and the importance of the timing of each recording were explained. Analysis of a post-hoc questionnaire of adherence to this timing showed that this procedure was followed satisfactorily.

Table 4.1. Final categories used for coding behaviours reported within the diaries.

\begin{tabular}{ll}
\hline 1. Sleeping & 13. Telephone use \\
2. Personal Care & 14. Homework \\
3. Eating/drinking & 15. Reading \\
4. At school & 16. Active hobbies \\
5. Motorised transport & 17. Cognitive hobbies \\
6. Active transport & 18. Unstructured play \\
7. TV/film watching & 19. Chores \\
8. Computer use & 20. Work \\
9. Computer gaming & 21. Sitting \\
10. Talking & 50. Sports \\
11. 'Hanging out' & 94. Behaviour missing \\
12. Listening to music & 95. Other \\
\hline
\end{tabular}


Table 4.2. Possible responses to the question 'Where are you?'

\begin{tabular}{ll}
\hline 1. Bedroom & 7. In town (inside) \\
2. Living room & 8. In town (outside) \\
3. Kitchen & 9. At school \\
4. Bathroom & 10. In a car, bus, train, taxi \\
5. Other room in my house & 11. Other inside area \\
6. Friend's house & 12. Other outside area \\
& 13. Garden \\
\hline
\end{tabular}

Table 4.3. Possible responses to the question 'Who are you with?'

\begin{tabular}{ll}
\hline 1. On my own & 5. With another (e.g., teacher or doctor) \\
2. With friends & 6. With friends and another \\
3. With family & 7. With family and another \\
4. With friends \& family & 8. With friends, family and another \\
\hline
\end{tabular}

All data was entered into SPSS Version 11.0. This allows data to be entered as either a numerical or string variable depending on whether it is to be analysed using quantitative or qualitative methods. The time sampling data from the diaries was analysed quantitatively and therefore was entered as numerical variables. This required that reported behaviour from participants be first placed into a numbered category similar to those given in Table 3.1. New categories were added and developed as behaviours were reported that were unaccounted for by the original list. The final list of behaviour categories is given in Table 4.1. Participants also reported where they were, and whom they were with by indicating one of the categories given in Tables 4.2 and 4.3 .

To allow comparison between participants' free-time sedentary behaviour, a summary variable of 'total sedentary behaviour' was calculated for both males and females. This was the total time spent watching television, using motorised transport, sitting 'doing nothing', talking, reading, listening to music, total computer use (work and games), and cognitive hobbies. These behaviours were chosen for inclusion because 
they are considered to be inactive from the combined definitions of Ainsworth et al. (2000) and Owen et al. (2000). Other sedentary activities, for example eating, personal care behaviours, and religious activities, were not included because they were not considered to be truly 'free-time'.

Total sleep time was also calculated and entered as a covariate to investigate whether individuals changed their behavioural choices during puberty, as hypothesised, or whether any increases in free-time sedentary behaviour were associated only with an increased availability of such time, for example, during evening time when previously they had gone to bed. Weekday and weekend data were analysed separately because it was considered that different patterns of behaviour might occur at the weekend compared with weekdays.

Additional information regarding the temperature and weather was collected before the start of each day. Weather was assessed on a 5-part scale from $1=$ Very cold to 5 $=$ Hot. Temperature was also assessed on a 5-point scale from $1=$ Snow to $5=$ Dry and probably will not rain.

\section{Body Composition}

For the anthropometric assessments participants were barefoot and dressed in light shorts and a t-shirt. For each dimension two measurements were taken; if the two measures differed by more than predetermined error limits (see Table 4.4) then a third measure was taken and the two closest measures used to calculate a mean value (Cameron, 1984). Intra- and interobserver reliability was assessed by calculation of the Standard Deviation of Differences $\left(S_{d}\right)$ and Standard Error of Measurement $\left(S_{e}\right)$ for each measurement site after the first 68 participants had been assessed (Cameron, 1984). The limits for each observer were then adjusted as shown in Table 4.5. 
Table 4.4. Measurement limits for anthropometric assessments.

\begin{tabular}{lc}
\hline & Limits \\
\hline Height & $+/-4 \mathrm{~mm}$ \\
Weight & $+/-0.4 \mathrm{~kg}$ \\
BIA & $+/-2 \%$ \\
Waist & $+/-1 \mathrm{~cm}$ \\
Iliac Crest & $+/-1 \mathrm{~cm}$ \\
Hip & $+/-1 \mathrm{~cm}$ \\
Triceps & $+/-1 \mathrm{~mm}$ \\
Biceps & $+/-1 \mathrm{~mm}$ \\
Subscapular & $+/-1 \mathrm{~mm}$ \\
Suprailiac & $+/-1 \mathrm{~mm}$ \\
\hline
\end{tabular}

Table 4.5. Reliability limits of anthropometric measurements.

\begin{tabular}{lcccccc}
\hline & \multicolumn{3}{c}{$\begin{array}{c}\text { Standard Deviation of } \\
\text { Differences }\end{array}$} & \multicolumn{3}{c}{ Measurement Limits $\left(\mathrm{S}_{\mathrm{e}}\right)$} \\
\cline { 2 - 7 } & Male & Female & All Data & Male & Female & All Data \\
\hline Height & 1.56 & 2.19 & 1.92 & $3(2)$ & $4(3)$ & $4(3)$ \\
Weight & 0.14 & 0.12 & 0.14 & $0.3(0.2)$ & $0.2(0.1)$ & $0.3(0.2)$ \\
BIA & 0.30 & 0.94 & 0.64 & 1 & 2 & 1 \\
Waist & 0.55 & 0.38 & 0.49 & $1.1(0.8)$ & $0.7(0.5)$ & $1.0(0.7)$ \\
Iliac Crest & 0.54 & 0.82 & 0.67 & $1.1(0.8)$ & $1.6(1.1)$ & $1.3(0.9)$ \\
Hip & 0.53 & 0.53 & 0.53 & $1.0(0.7)$ & $1.0(0.7)$ & $1.0(0.7)$ \\
Triceps & 0.57 & 0.64 & 0.60 & $1.1(0.8)$ & $1.2(0.8)$ & $1.2(0.8)$ \\
Subscapular & 0.54 & 0.40 & 0.51 & $1.1(0.8)$ & $0.8(0.6)$ & $1.0(0.7)$ \\
Biceps & 0.57 & 0.51 & 0.55 & $1.1(0.8)$ & $1.0(0.7)$ & $1.1(0.8)$ \\
Suprailiac & 0.71 & 1.24 & 0.98 & $1.4(1.0)$ & $2.4(1.7)$ & $1.9(1.3)$ \\
\hline
\end{tabular}

Stature was measured using a Holtain stadiometer to the last completed $0.1 \mathrm{~cm}$; participants stood upright with their heels and buttocks touching the backboard, and the heels together. The head was positioned in the 'Frankfurt Plane', the headboard lowered to the vertex of the skull, and a $0.5 \mathrm{~kg}$ weight placed on the top of the headboard to flatten the participant's hair; a second observer checked that their heels stayed in contact with the floor. The participant took a deep breath and then exhaled, the maximum observed reading to the last completed unit was recorded as their height (Cameron, 1984). Body mass was obtained from Tanita TBF 611 scales (Tanita UK Ltd, Middlesex, UK) to the nearest $0.2 \mathrm{~kg}$. BMI and BMI centile status were calculated from the height and weight data and Cole et al.'s (1995) UK reference 
curves. The Tanita TBF 611 scales were also used to assess percentage body fat from BIA.

After marking each site, triceps, biceps, subscapular, and suprailiac SKF measurements were taken on the right side of the body using Holtain callipers according to the methodology of Cameron (1984):

Triceps - midway between the acromion and the olecranon, on the posterior vertical axis halfway between the lateral and medial surfaces of the arm. The skinfold was picked up $1 \mathrm{~cm}$ above the marked midpoint with the arm flexed to $90^{\circ}$. The participant then extended the arm so that it hung relaxed by their side and the callipers applied at the marked point. The calliper reading was taken three seconds after release.

Subscapular - the site was marked $1 \mathrm{~cm}$ below the inferior angle of the scapular with the participant standing with shoulders relaxed and the arms hanging loosely at the sides. The skinfold was picked up at a lateral, downwards angle to follow the natural grain of the body fat.

Biceps - midway between the acromion and the olecranon, on the anterior vertical axis halfway between the lateral and medial surfaces of the arm. The skinfold was taken as for the triceps.

Suprailiac - taken in the mid-axillary line $1 \mathrm{~cm}$ above the anterior superior iliac spine. This vertical skinfold was taken with the participant placing the right hand on the left shoulder so that the arm did not impede the observer.

Fat distribution was assessed via measurements of hip (maximum extension of the buttocks) and waist (narrowest part of the torso) circumferences to the nearest $0.1 \mathrm{~cm}$, a measurement at the iliac crest was also taken in order to have an immovable

\footnotetext{
1 After the Phase 1 assessments it became apparent that for BIA the Tanita TBF 611 scales gave erroneous values for the sample under study because they were designed for use with adults only and calculated \%BF using adult specific equations. BIA was therefore removed from all analyses and not used in Phases 2 and 3.
} 
reference point for future assessments. For measurements of the waist and iliac crest the student stood facing the observer with the arms hanging at the sides, for the hip measure the observer kneeled down at the side in order that the buttocks extension could be clearly seen. In each case the tape was placed around the torso in a horizontal plane without compressing the skin and a second observer helped to check its correct positioning (Lohman et al., 1988).

\section{Physical Self-Perception}

Body image was assessed using a shortened version of the Physical Self-Perception Profile for Children (C-PSPP (Whitehead, 1995)). The scales included were global self-worth, physical self-worth, and body attractiveness. For this study, perceptions of body attractiveness were used as the measure of body image.

\section{Pubertal Status}

One section of questions included within the free-time diary aimed to elicit a selfrating of pubertal status from each individual. In concordance with the theory described above, this relatively simple process was designed to divide the cohorts into four divisions: pre-pubertal, early-pubertal, late-pubertal, and adult.

Participants were asked to complete the following questions:

1. Have you started puberty? (e.g., do you have any pubic hair?)

2. Girls only

a. Have you begun to menstruate? (i.e. do you have periods?)

b. If yes - when did you have your first menstruation/period?

\section{Boys only}

a. Has your voice broken? (i.e. do you speak with a deeper voice than when you were younger?)

b. If yes - when did you first notice that your voice was deeper?

c. Have you started to shave?

d. If yes - how many times per month do you shave? 
During the Phase 1, cross-sectional analysis, pre-pubertal participants were those who answered negatively to question 1 i.e. they had no pubic hair. Early-pubertal participants were those who had pubic hair but for whom menarche or voice breaking had not yet occurred. If the responses to questions 1 and $2 \mathrm{a}$ were both positive then participants were late-pubertal if menarche or voice breaking had occurred in the previous 24 months, or post-pubertal if menarche or voice breaking had occurred more than 24 months previously. Questions $2 \mathrm{c}$ and $2 \mathrm{~d}$ for the boys used facial hair growth as an extra indication of maturity where other information was not available.

In addition to self-assessment of pubertal status, researchers also assessed the boys during the taking of anthropometric measures. Three questions were answered either 'yes' or 'no':

$$
\begin{aligned}
& \Rightarrow \text { Has the boy's voice broken? } \\
& \Rightarrow \text { Does he have underarm (axillary) hair? } \\
& \Rightarrow \text { Does he have facial hair? }
\end{aligned}
$$

It was necessary to assess pubertal status on two counts, firstly to discover any associations between puberty development and changes in inactivity patterns; but also to calculate the boys' percentage body fat from skinfolds because the Slaughter (1988) equations require males to be defined as pre-pubertal, pubertal, or adult (no similar adjustment is required for females). However, it was possible for self-assessment and researcher-assessment to differ, particularly regarding whether or not the participant's voice had broken. Where this occurred the student's assessment was used to classify him as early- or late-puberty because it was felt that they would have had a clearer idea of any changes that may have occurred.

During Phase 1, in the majority of cases where this occurred (7/9) the boys had rated 'yes' compared to the researchers 'no', in one case a boy had rated 'no' when the researcher had rated 'yes' and in one case a boy had rated 'not sure' when the researcher had rated 'yes' (in this case the researcher's assessment was taken). The effect this had was to classify eight boys as late- rather than early-pubertal and one as early- rather than late-pubertal. This made no difference in the calculation of \%BF because the categories 'early-pubertal' and 'late-pubertal' were collapsed into one 
category 'pubertal'. There may, however, have been a consequence when comparing changes in pubertal state with changes in behaviour.

During the longitudinal analysis, voice-breaking was substituted by axillary hair growth when classifying the boys' pubertal status. The reason for this was that during the collection of data from Phases 2 and 3 it became apparent that the boys had difficulty in consistently assessing if and when voice breaking had occurred. However, because no data had been collected on when hair growth was first observed, it was decided to classify the boys as follows:

Pre-pubertal participants were those who had no pubic hair. Early-pubertal participants were those who had pubic hair but for whom axillary hair had not yet appeared. Participants who had both pubic and axillary hair were classified as latepubertal/adult. Additionally, in order to be consistent between the genders, the female categories of late-pubertal and adult were also analysed as one.

\section{Statistical Analyses}

a) Phase 1 (cross-sectional) analyses

All analyses were carried out using SPSS v11.0 for Windows. Cronbach's $\alpha$ was used to test the internal reliability of the Physical Self-Perception Profile for Children (CPSPP). The effects of pubertal status on sedentary behaviour were analysed using independent-samples $\mathrm{t}$-tests. Analysis of variance was used to test for the effect of school year group and to examine differences in sleep time between pubertal stages and between age groups. After means of sedentary time had been adjusted for sleep time, analysis of covariance was used to test for differences between groups. Post hoc Bonferroni tests were used to examine the source of any differences. Partial correlation coefficients controlling for sleep were calculated to examine the relationships between puberty, body composition, body image and sedentary behaviour. Kendall's partial $\tau$ correlation coefficient was used for non-parametric data. One-tailed tests of significance were used where previous research allowed the expected direction of correlation to be specified in advance. In other cases two-tailed tests of significance were used. Weekend data were not included in the analysis 
because it was considered that for a small sample one day of recording was not enough to obtain an indication of habitual behaviour.

Analyses were carried out separately for males and females because previous research has found differences in inactivity between the genders (Bradley et al., 2000; Janz et al., 2000).

\section{b) Longitudinal analyses}

All analyses were carried out using SPSS v11.0 for Windows. For repeated measures, correlated scores analysis of variance was used with data that satisfied the requirement of normality. Within the normal data, if the assumption of sphericity was violated then the degrees of freedom were adjusted using the Huynh-Feldt and Greenhouse-Geisser estimates according to the advice given by Field (2000). Post-hoc analyses were obtained by comparing the main effects using a Bonferroni adjustment. For nonnormal data, The Friedman Test for three or more related samples was used.

Analysis of covariance was used to test for any differences between students who changed pubertal status and those who did not. Phase 1 total sedentary behaviour and change in total sleep time were entered as covariates in order to control for their effects. Preliminary analyses had shown that Phase 1 pubertal status had no effect as a covariate and it was not included. Non-normal data were log-transformed, if the data were still non-normal after transformation then any outliers greater than two standard deviations from the mean were examined to see if deleting them returned the data to normality. This criterion resulted in one girl being deleted from the weekday analysis plus one boy and one girl being deleted from the weekend day analysis. In neither case did the deletion of any outlier affect the significance of the outcome.

Hierarchical multiple regression was used to test for the effects of change in pubertal status, change in body composition including fat distribution (BMI, BMI centile, \% body fat, waist-hip ratio), and change in body image when predicting the change in total sedentary behaviour, after controlling for Phase 1 sedentary behaviour and change in total sleep time. As before, Phase 1 pubertal status was not included as a predictor as preliminary analyses had shown it to have no effect. Non-normal data 
were log-transformed. Residual outliers were identified as cases in which the standardized residual was greater than 2 . In these cases the Cook's distance, central leverage value, and Mahalonobis distance were examined in order to assess the influence of the case on the model. If any of the values exceeded the limits suggested in Field (2000) then the case was considered to be too great an influence and was deleted before reanalysing the data. 


\section{Chapter 5: Participants}

\section{Power Calculation for Estimating Required Sample Size}

A pre-assessment power analysis was performed in order to determine the necessary number of participants required. Calculations were performed for males and females separately using the data for baseline and 6-month assessment ages of each group. The variable used for the calculations was BMI because of its importance to the hypothesis of the study and the availability of large-scale representative data.

Using the data of Cole et al. (1995), age specific means and standard deviations for BMI were obtained at six month intervals from 10 to 15 years of age. Mueller, Dai and Labarthe (2001) have previously calculated 1-year BMI tracking correlations for 8-, 11-, and 14-year-olds and these figures were also used in the analysis. Following the method of Howell (1997) for a matched-sample, $\alpha$ was set at .05 and power (1- $\beta$ ) was set at .80. Example calculations are set out below:

Effect size $\mathrm{d}=\left(\mu_{1}-\mu_{2}\right) / \sigma(2(1-\rho))^{1 / 2}$

Where $\mu_{1}-\mu_{2}$ represents the expected difference in the two population means, $\sigma$ is the population standard deviation (assuming homogeneity of variance $\sigma_{X 1}^{2}=\sigma_{X 2}^{2}=\sigma^{2}$ ), and $\rho$ is the population correlation between the two means $\mathrm{X} 1$ and $\mathrm{X} 2$.

Also, the sample size $\mathrm{N}=(\delta / \mathrm{d})^{2}$, where $\delta$ is the noncentrality parameter equal to 2.8 for a two-tailed test with $\alpha=.05$, and power $=.80$.

For males aged 11 years at baseline:

$\mathrm{d}=(17.154-16.892) / 1.91(2(1-0.97))^{1 / 2}=0.56$

$\mathrm{N}=(2.80 / 0.56)^{2}=\underline{\underline{25.00}}$ students required.

For females aged 11 years at baseline:

$\mathrm{d}=(17.793-17.478) / 2.25(2(1-0.96))^{1 / 2}=0.49$

$\mathrm{N}=(2.80 / 0.49)^{2}=\underline{\underline{32,65}}$ or 33 students required. 
Similar calculations indicated that 38 boys and 19 girls were required at age 13, and 43 boys and 33 girls were required at age 15. For a one-tailed test with $\alpha=.05$ and power $=.80 ; \delta=2.50$ and the required sample sizes were 20 boys and 27 girls at age 11,30 boys and 15 girls at age 13, and 34 boys and 27 girls at age 15 .

\section{Recruitment}

Two schools from northwest Leicestershire took part in the study. These schools were chosen because of their intake age range (10 - 17 years), which allowed access to participants throughout the pubertal period. Informed consent was obtained from both the child and a parent or carer. The study received ethical approval from Loughborough University's Ethical Advisory Committee.

Students in Year 6 (10.0 - 10.9 years of age at intake; Cohort A), Year 8 (12.0 - 12.9 years; Cohort C), and Year 10 (14.0 - 14.9 years; Cohort E) were provided with detailed information and invited to participate. Incentives (approximate value $\mathfrak{1 5}$ ) were offered for students who volunteered and completed the phase. Of the 133 students who initially volunteered 119 completed Phase 1. In Phase 2, 104 students completed all tasks of whom 100 had also completed Phase 1. Sixty of those who completed were boys and 44 were girls. In Phase 3, 84 students completed the phase. In total, 83 students completed enough of the data to be included in the longitudinal analysis, 43 boys and 40 girls. 


\section{Chapter 6: Phase 1 (Cross-Sectional)}

\section{$\underline{\underline{\text { Results }}}$}

\section{$\underline{\text { Reliability testing }}$}

The $\alpha$ reliability of Physical Attractiveness from the C-PSPP was .80 for males and .88 for females, indicating that the scale that was used to determine body image had good reliability. These are within the ranges reported by Whitehead (1995).

\section{Pubertal Status}

Of the 64 boys who completed this phase, 23 were pre-pubertal, 15 were earlypubertal, 18 were late-pubertal and 8 were post-pubertal. The corresponding numbers of girls were 12 pre-pubertal, 12 early-pubertal, 25 late-pubertal, and 6 post-pubertal.

\section{Behaviour differences}

a) Absolute sedentary time

The different effects of (1) the onset of pubertal change and (2) increasing maturity were analysed by grouping the sample according to pubertal status: pre-pubertal versus pubertal/post-pubertal, and pre-/early-pubertal versus late-/post-pubertal. These effects can be seen in Table 6.1.

The total amount of time spent in sedentary behaviours was significantly higher $(\mathrm{p}<$ .01) for both females (mean difference 63 minutes) and males (mean difference 53 minutes) who had already started puberty compared with those who were prepubertal. Additionally, the total amount of time spent in sedentary behaviours was significantly higher $(p<.05)$ for both females (mean difference 37 minutes) and males (mean difference 43 minutes) who were late- or post-pubertal compared with those who were pre- or early-pubertal. 
The effect of age (school year group) can also be seen in Table 6.1. Significant differences in the total amount of time spent in sedentary behaviours existed between Year 10 and Years 6 and 8 for both the boys and the girls, with Year 10 students spending more time being sedentary.

\section{b) Sleep time}

Table 6.2 presents results for total weekday sleep time. Significant year group and puberty effects were noted. Participants in higher year groups spent less time in bed sleeping than their younger peers, and, in addition, participants who were late- or post-pubertal spent less time in bed than those who were pre-pubertal (boys and girls) or early-pubertal (boys only).

\section{c) Sedentary time after controlling for sleep}

The effect of adjusting sedentary behaviour for sleep time is shown in Table 6.3. This adjustment had a significant effect because differences between groups for onset or increased puberty were removed. In addition, the age effect for girls was also removed. However, for boys, a significant difference between Year 10 and Years 6 and 8 was still present. This difference remained significant after pubertal status was also entered as a covariate $(F=5.7, p<.01)$.

\section{d) Puberty, body composition, body image, and sedentary behaviour}

After controlling for sleep time, partial correlations between pubertal status, measures of body composition and fat distribution, body image, and sedentary behaviour showed that, for boys, both age and pubertal status correlated significantly with BMI $(\mathrm{r}=.32, \mathrm{p}<.01 ; \mathrm{r}=.29, \mathrm{p}<.01 ;$ both 1-tailed $)$ and waist-hip ratio $(\mathrm{r}=-.28, \mathrm{p}<.01 ; \mathrm{r}$ $=-.22, \mathrm{p}<.01$; both 1-tailed) but not BMI centile or percentage body fat. Body composition measures, BMI $(r=-.17, p<.05 ; 1$-tailed $)$, BMI centile $(r=-.16, p<.05$; 1 -tailed), and percentage body fat $(r=-.19, p<.05 ; 1$-tailed $)$ correlated significantly with body image, but fat distribution (waist-hip ratio) did not. For girls, pubertal status correlated significantly with BMI $(r=.44, p<.001 ; 1$-tailed $)$, BMI centile $(r=.27, p$ 
$<.01 ; 1$-tailed), percentage body fat $(\mathrm{r}=.27, \mathrm{p}<.01 ; 1$-tailed), and waist-hip ratio ( $\mathrm{r}=$ $-.19, \mathrm{p}<.01 ; 1$-tailed). In addition, body image also correlated significantly with BMI ( $r=-.53, p<.001 ; 1$-tailed), BMI centile $(r=-.49, p<.001 ; 1$-tailed), and percentage body fat $(r=-.29, p<.001 ; 1$-tailed) but not waist-hip ratio. Only BMI $(r=.32, p<$ $.05 ; 1$-tailed $)$ and waist-hip ratio $(r=-.23, \mathrm{p}=.05 ; 1$-tailed $)$ correlated significantly with age.

For both genders, correlations with sedentary behaviour were infrequent. BMI was the only variable that correlated significantly for both boys $(\mathrm{r}=.30, \mathrm{p}<.01 ; 1$-tailed $)$ and girls $(r=.32, p<.05 ; 1$-tailed). Body image could only approach significance for girls $(r=-.23, p=.054 ; 1$-tailed). Age was the most strongly correlated but for boys only ( $r$ $=.36, \mathrm{p}<.01 ; 2$-tailed). 
Table 6.1. Mean (SD) of time in minutes per day spent in sedentary behaviours by pubertal group and school year group.

\begin{tabular}{|c|c|c|c|c|c|c|}
\hline & \multicolumn{4}{|c|}{ Pubertal status } & \multicolumn{2}{|c|}{$\begin{array}{l}\text { F-values } \\
\text { (parentheses show combination of } \\
\text { groups for comparison) }\end{array}$} \\
\hline & 1. Pre- & 2. Early- & 3. Late- & 4. Post- & Puberty & Post-Hoc \\
\hline Boys' sedentary & $176(66)$ & $218(70)$ & $237(91)$ & $234(70)$ & $7.6 * *$ & $1<(2,3,4)$ \\
\hline behaviour & {$[n=23]$} & {$[n=15]$} & {$[\mathrm{n}=18]$} & {$[\mathrm{n}=8]$} & $5.1^{*}$ & $(1,2)<(3,4)$ \\
\hline \multirow{4}{*}{$\begin{array}{r}\text { Girls' sedentary } \\
\text { behaviour }\end{array}$} & $176(49)$ & $233(67)$ & $235(73)$ & $269(62)$ & $8.6 * *$ & $1<(2,3,4)$ \\
\hline & {$[\mathrm{n}=12]$} & {$[n=12]$} & {$[\mathrm{n}=25]$} & {$[\mathrm{n}=6]$} & $4.0^{*}$ & $(1,2)<(3,4)$ \\
\hline & \multicolumn{4}{|c|}{ School Year Group } & \multicolumn{2}{|l|}{ F-values } \\
\hline & Year 6 & Year 8 & \multicolumn{2}{|r|}{ Year 10} & Year & Post-Hoc \\
\hline \multirow{2}{*}{$\begin{array}{r}\text { Boys' sedentary } \\
\text { behaviour }\end{array}$} & $169(61)$ & $200(70)$ & & $267(73)$ & \multirow{2}{*}{$11.4^{* * *}$} & \multirow{2}{*}{$6,8<10$} \\
\hline & {$[n=22]$} & {$[n=22]$} & & {$[n=20]$} & & \\
\hline \multirow{2}{*}{$\begin{array}{r}\text { Girls' sedentary } \\
\text { behaviour }\end{array}$} & $202(64)$ & $216(59)$ & & $286(76)$ & \multirow{2}{*}{$6.4^{* *}$} & \multirow{2}{*}{$6,8<10$} \\
\hline & {$[n=18]$} & {$[n=26]$} & & {$[\mathrm{n}=11]$} & & \\
\hline
\end{tabular}

${ }^{*} p<.05,{ }^{* *} p<.01,{ }^{* * *} p<0.001$ 
Table 6.2. Mean (SD) of sleep time in minutes per 24-hour period by pubertal status and school year group.

\begin{tabular}{|c|c|c|c|c|c|c|}
\hline & \multicolumn{4}{|c|}{ Pubertal status } & \multicolumn{2}{|l|}{ F-values } \\
\hline & $\begin{array}{l}\text { (1) Pre- } \\
\text { pubertal }\end{array}$ & $\begin{array}{l}\text { (2) Early- } \\
\text { pubertal }\end{array}$ & $\begin{array}{l}\text { (3) Late- } \\
\text { pubertal }\end{array}$ & $\begin{array}{l}\text { (4) Post- } \\
\text { pubertal }\end{array}$ & Puberty & Post-Hoc \\
\hline $\begin{array}{r}\text { Boys' total weekday } \\
\text { sleep time }\end{array}$ & $583(38)$ & $576(31)$ & $526(37)$ & $518(43)$ & $12.4^{* * *}$ & $1,2>3,4$ \\
\hline \multirow[t]{3}{*}{$\begin{array}{r}\text { Girls' total weekday } \\
\text { sleep time }\end{array}$} & $594(49)$ & $551(44)$ & $537(40)$ & $513(36)$ & $6.6 * * *$ & $1>3,4$ \\
\hline & \multicolumn{4}{|c|}{ School Year Group } & F-values & \\
\hline & Year 6 & Year 8 & & Year 10 & Year & Post-Hoc \\
\hline $\begin{array}{r}\text { Boys' total weekday } \\
\text { sleep time }\end{array}$ & $589(32)$ & $560(39)$ & & $519(38)$ & $19.4^{* * *}$ & $6>8>10$ \\
\hline $\begin{array}{r}\text { Girls' total weekday } \\
\text { sleep time }\end{array}$ & $580(54)$ & $550(34)$ & & $501(28)$ & $12.8 * * *$ & $6>8>10$ \\
\hline
\end{tabular}


Table 6.3. Adjusted mean (SE) of time in minutes per day spent in sedentary behaviours with sleep as a covariate.

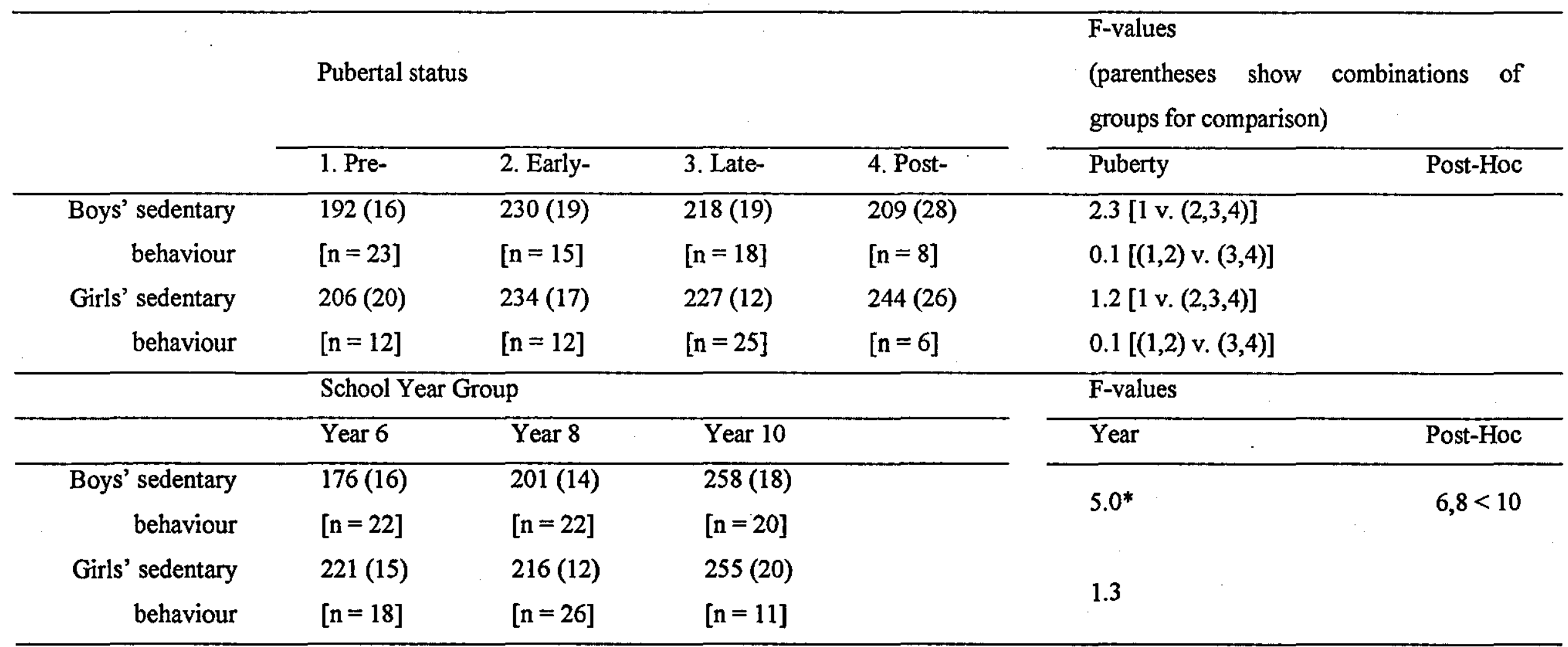

${ }^{*} p<0.05$ 


\section{Chapter 7: Phase 1 Discussion}

The hypothesis of this study was that time spent in sedentary behaviours would be greater in participants with more advanced pubertal status, and that body composition and body image would be mediators of this difference via differences in behavioural choices. The initial comparison between pre-pubertal students and those who were pubertal or post-pubertal showed that, for participants who had already started puberty, weekday sedentary behaviour was greater. To test for the effect of advancing development, the pre- and early-pubertal participants were compared with those who were late- or post-pubertal. These results also showed greater sedentary behaviour among the more advanced participants, thus supporting the results of Bradley et al. (2000) that sedentary behaviours increase during adolescence. In contrast to Bradley et al., who only found an effect in girls, the current study found an effect in both genders. The differences may be due to differing methods of data collection. In the earlier study, annually administered questionnaires were used to assess each participant's three most commonly performed activities. Adult MET values (in the absence of available values for children) of $2,3,5$, or 8 METs were used to assign each activity a level of intensity. Sedentary students were those who reported at least two of the three activities with METs of 2 or 3 . This is a higher value compared with other definitions (Ainsworth et al., 2000; Owen et al., 2000) and higher than the METs associated with any of the behaviours used to, estimate sedentary time in the current study. This may have led to an over-reporting of sedentary behaviour in the study by Bradley et al.

Analysis between year groups showed that the oldest participants spent more time in sedentary behaviours compared with those in lower year groups. These findings are supported by those of previous studies. Bradley et al. (2000) reported that sedentary activities were more frequently reported with increasing age among a group of 656 American students aged 8 - 15 years, and Deheeger et al. (2002) found that for 94 French adolescents aged $10-16$ years time watching TV/computer use increased with age from 1.4 (boys) and 1.2 (girls) hours per day at age 10, to 1.8 (boys) and 1.6 (girls) hours at age 14, and 2.2 (boys) and 1.9 (girls) hours at age 16. 
In contrast, Myers et al. (1996) reported that total hours of television viewing (before and after school) showed no grade effect. A finding supported by the studies of Lawrence et al. (1986), Shann (2001), and Wolf et al. (1993). A possible explanation for the differences between the results of these studies and those of the current study may be the more comprehensive measure of sedentary behaviour used by Project STIL compared with the single behaviour used by the others.

Time spent sleeping was introduced into the analysis because the older participants appeared to be completing the diary for longer each day than their younger counterparts. This finding is supported by earlier research that has shown weekday sleep time to be dependant on age (Laberge et al., 2001; Wolfson \& Carskadon, 1998) with older adolescents reporting later bedtimes, less total time in bed, and having, therefore, more available free-time particularly in the evening when behaviours are more likely to be sedentary. The results presented here supported such patterns.

The effect of introducing sleep as a covariate was to remove all significant differences between pubertal groups. This suggests that differences in sedentary time during adolescence were not associated with the shifting costs of sedentary versus active behaviours, but to a lack of available alternative behaviours during later waking hours. In other words, more advanced adolescents were not choosing more sedentary behaviours in preference to others, rather they continued such behaviours for longer into the evening. The importance of this finding is that it brings into question previous research that has found sedentarism to be associated with maturity (Bradley et al., 2000). Such conclusions have been made without taking the time available for such behaviour into account.

A different effect was seen when sleep was introduced into the analysis between year groups. Although significant differences were removed from the girls' results, boys still showed greater time spent in sedentary behaviour in Year 10 compared with both Year 6 and Year 8, independent of pubertal status. A possible explanation for this may be the change of schools that occurred for these students after Year 9. Under the local schooling system students move from high school to upper school at the start of Year 10. This means that they have the opportunity to change friendship groups as a 
number of high schools provide students for each upper school. This change of environment may have affected participants' behaviour. The bigger effect seen for boys compared with girls may have been because of the lower values for boys in Years 6 and 8 compared with girls. Although differences between the genders were not significant within each year group, the data do show boys to have had less sedentary time during Years 6 and 8 and a subsequently bigger difference between Years 8 and 10 when they caught girls up.

Relationships between puberty and body compositional variables were consistent with the findings of previous research (Rogol et al., 2002). BMI and waist-hip ratio were significantly correlated with puberty for both genders but a relationship between percentage body fat and puberty was found for girls only. This may be due to changes in boys' percentage body fat that are curvilinear during puberty. A relationship was also found between pubertal status and BMI centile for girls, which suggests that some of the girls had started puberty at an earlier age than others. BMI centile is an age-related construct that reflects an individual's height related body mass in relation to their peers and earlier maturing girls will increase body mass at a greater rate. The lack of a similar finding in boys may have been a result of a greater fraction of the boys still being pre- or early-pubertal and not yet changing body composition significantly more than their peers.

Relationships between body composition and body image were also consistent with previous research (Duncan, Woodfield, ONeill, \& Al-Nakeeb, 2002; Kimm et al., 1997; Kolody \& Sallis, 1995; Sands \& Wardle, 2003) with both boys and girls reporting a more negative body image with higher percentage body fat, BMI, and BMI centile. Duncan et al. found correlations between percentage body fat and body image to be $r=-.66$ for boys and $r=-.62$ for girls aged $11-14$ years. However, the current study found correlations to be stronger in girls compared with boys. This may be a result of differing demographics between the samples used in each study. For example, the current study used a sample that predominantly consisted of White participants whereas Duncan et al. used a more multi-racial cohort. As correlations for White participants $(r=-.62)$ were shown to be different to those for Black $(r=-.72)$ and Asian $(r=-.60)$ participants this may have had an effect when all ethnic groups were analysed together by gender. 
In contrast to the current study, McCabe and Ricciardelli (2003) found weak, but significant, positive relationships between body image satisfaction and body composition for Australian adolescent boys $(\beta=.20, p<.01)$ and girls $(\beta=.15, p<$ .05) from Grades $7-10$ (mean ages 14.02 years and 13.92 years respectively). The explanation for these results may lie in the use of BMI as a measure of body composition. Because BMI is a measure of weight for height, rather than actual body composition, greater BMI may be due to increased muscle rather than fat, which is in line with the desired sociocultural body image in Australia (M. McCabe, personal communication, 11 April 2003). This is an unproven hypothesis but may merit further investigation.

No relationships were found between waist-hip ratio and body image for either the boys or the girls. This is in contrast to the ideas of Page and Fox (1997) particularly for the girls. However, for this sample it appears that overall body composition was more dominant than the distribution of the body fat in the formation of the perception of body attractiveness.

Previous research has shown relationships between BMI and sedentary behaviour in adults (Martinez-Gonzalez, Martinez, Hu, Gibney, \& Kearney, 1999). Maffeis (1998) found a relationship between relative BMI (BMI/BMI at $50^{\text {th }}$ centile) and television watching in children ( 8 years old), but not adolescents (12 years old), and Kolody and Sallis (1995) reported that an increase in BMI was associated with an increased preference for watching television compared with walking or playing a running game with friends. Although the current study found significant but weak relations between sedentary behaviour and BMI, when BMI centiles were substituted in place of BMI these relationships disappeared. For boys this can be explained by the strength of the age-sedentary behaviour correlation $(r=.360, p<.01)$, which suggests that the result seen for BMI was moderated by the effect of age. This finding is harder to explain for girls for whom the age-sedentary behaviour relationship was much weaker $(r=.087, p$ $=.27)$. However, the BMI centile-sedentary behaviour relationship was stronger $(r=$ $.195, \mathrm{p}=.085)$, compared with that for boys, and more closely approaching significance. This relationship may therefore be more important than found here and one that requires more attention in future studies. 
Overall the relationships between body composition and sedentary behaviour were weak, with, for example, no significant correlations found between percentage body fat and sedentary behaviour. However, Gortmaker et al. (1996) found that television affects obesity in youth. Taken together, these results may suggest that television more than total sedentary time is influenced by other obesogenic factors such as snacking. In other words, increased energy input rather than reduced energy expenditure is responsible for a change in body composition. This is an area for future research. The alternative explanation for the relationship found in the current study, that body composition has a weak effect on sedentary behaviour perhaps via body image as hypothesised, may be rejected by the lack of significant correlations between body image and sedentary behaviour.

In fact the lack of behavioural choice difference that was found in girls may have been due to the weakness of any relationship between body image and sedentary behaviour because correlations between pubertal status and body composition, and body composition and body image were relatively strong. For boys, however, relations between pubertal status, body composition and body image were less consistent. Overall, a significant correlation was seen between age and sedentary behaviour but not pubertal status and sedentary behaviour. Bradley et al. (2000) also found an increase in boys' sedentary behaviour to be related to age but not pubertal status in a sample of 325 boys of ages $8-15$ years. This suggests that, for boys, determinants of sedentary behaviour exist that may be controlled by age based changes rather than pubertal changes. The weakness of the hypothesised pubertal status-body composition-body image relationship that was seen in boys supports this theory. Such determinants should be the focus of future research.

One note of caution, during the analyses of covariance the assumption of homogeneity of regression was violated in each case. This was because not all of the correlations between sleep and sedentary time were equal at all levels of pubertal status or age. Although the relationships were all negative some failed to reach significance. This was possibly due to too few students and it is therefore recommended that future studies try to replicate these results with greater numbers of participants. 


\section{Chapter 8: Longitudinal Results}

\section{$\underline{\text { Participants }}$}

In Phase 1, 119 participants completed all tasks out of the 133 who initially volunteered to take part. No significant differences were found for anthropometric measures or body image between those who completed the phase and those who did not. In Phase 2, 104 participants completed all tasks of whom 100 had also completed Phase 1. Univariate ANOVA showed no differences in body composition or time spent in sedentary behaviours between those who completed Phase 1 but not Phase 2 . An independent samples t-test found that participants who started but did not complete Phase $2(n=14)$ had significantly lower levels of percentage body fat than those who did complete $(7.7 \%$ versus $19.9 \%, p=.001)$. No significant differences were found for any other anthropometric measures or body image. Eighty-three participants had enough completed data to be included in the longitudinal analyses; that is at least Phases 1 and 3 weekdays completed. Comparisons between those who completed the whole study and those who dropped out after Phase 1 showed that boys who dropped out tended to be at a greater stage in their pubertal development than those who completed $(p<.01)$. No other differences were found. Of the 83 participants, 1 boy and 3 girls did not include Phase 2 data. Two boys and two girls did not include enough weekend data.

\section{Repeated Measures}

Repeated measures analysis was used to ensure that the variables of interest followed expected patterns of change over the three phases i.e. there was no indication that the data in any particular phase was unusual. The effect of increasing age (assessed as phase) by cohort on the key variables can be seen in Tables 8.1 and 8.2. No effect was seen for body image, weekday sedentary behaviours or weekend sleep for either boys or girls $(\mathrm{p}>.05)$. Weekend sedentary behaviours showed a significant age effect for Cohort $\mathrm{A}$ boys only with a post-hoc analysis indicating that the time spent being 
sedentary during Phase 2 was significantly greater than in Phase 3. Weekday sleep showed a significant negative effect of age for Cohort A girls only.

Age was found to have a significant positive effect on boys' BMI for all three cohorts. For girls' BMI, age had a significant positive effect in Cohorts $\mathrm{A}$ and $\mathrm{C}$ but not Cohort E. Percentage body fat was seen to have a significant effect of age for boys in Cohorts $\mathrm{C}$ and $\mathrm{E}$. For Cohort $\mathrm{C}$ boys, \%BF was significantly lower in Phase 3 compared with Phases 1 and 2, but for boys in Cohort E, \%BF was significantly lower in Phase 2 compared with Phases 1 and 3 . Age had an effect on percentage body fat for girls in Cohort $\mathrm{C}$ only, the measure during Phase 1 being significantly lower than in Phases 2 and 3. The waist-hip ratio for girls was also changed significantly during the three phases in Cohort $\mathrm{C}$, the Phase 3 measure being significantly lower than that in Phases 1 and 2.

Analysis of the self-assessed temperature during each phase (not shown in Tables 8.1 and 8.2) showed boys in Cohort A rated the Phase 3 weekend temperature as higher than that of Phase 2 and Phase $1(p<.05)$, and girls in Cohorts $A$ and $C$ rated the Phase 2 temperature as the worst $(p<.05)$. Differences were also found in the assessments of weekday temperature at each phase. Both genders in Cohorts A and C rated the Phase 2 weekday temperature as the lowest $(p<.01)$. No differences were found between phases in the assessments of Cohort $E(p>.05)$. 
Table 8.1. Boys' descriptive data for longitudinal analysis. Values are means (standard deviations).

\begin{tabular}{|c|c|c|c|c|c|c|c|c|c|}
\hline \multirow[b]{3}{*}{ Age } & \multicolumn{3}{|c|}{ Cohort A $(\mathrm{N}=18)$} & \multicolumn{3}{|c|}{$4 \cup, 4 \cup($ Cohort C $(\mathrm{N}=14)$} & \multicolumn{3}{|c|}{ Cohort E $\mathrm{N}=11):$ ) } \\
\hline & Phase 1 & Phase 2 & Phase 3 & $\$$ Phase 1 & 10 Phase 2 & Phase 3 & Phase ! & Phase 2.: & Phase 3 \\
\hline & $11.2(0.4)$ & $11.7(0.3)$ & $12.1(0.3)$ & $131(03)$ & $137(03)$ & $142(03)$ & $150(0.8)$ & $15.5(0.3)$ & $160(0.3)$ \\
\hline BMI & $17.9(3.3)$ & $18.1(3.4)$ & $18.3(3.3)^{*}$ & $186(20)$ & $190(22)$ & $19: 4(21)^{* * *}$ & $20.6(3.3)$ & $209(29)$ & $213(32)) * *$ \\
\hline SKF \% & $18.9(7.2)$ & $19.3(8.4)$ & $19.1(7.2)$ & $211(90)$ & $189(79)$ & $165(5.4)^{* * 1}$ & $149(3,6)$ & $13.4(4) 1)$ & $14,6(4,2)^{* *}=$ \\
\hline $\begin{array}{l}\text { Waist-Hip } \\
\text { Ratio }\end{array}$ & $.811(.028)$ & $.805(.030)$ & $.803(.027)$ & $805(025)$ & $802(032)$ & $794(033)$ & $768(042)$ & $777(036)$ & $776(039)$ \\
\hline $\begin{array}{l}\text { Body } \\
\text { Image } \\
\end{array}$ & $2.7(0.6)$ & $2.7(0.4)$ & $2.8(0.4)$ & $26(0.5)$ & $28(0.6)$ & $29(06)$ & $2.4 .0 .5)$ & $2.5(0.6)$ & $2.4(0.5)$ \\
\hline \multicolumn{10}{|c|}{ Weekday time $\mathrm{min} / \mathrm{d}$} \\
\hline $\begin{array}{l}\text { Sedentary } \\
\text { behaviours }\end{array}$ & $177(57)$ & $200(57)$ & $179(67)$ & $218(73)$ & $242(70)$ & $254(97)$ & $268(54)$. & $242(50)$ & $237.87)$ \\
\hline Sleep & $590(35)$ & $591(33)$ & $597(44)$ & $566(44)$ & $557(33)$ & $556(48)$ & $517(40)$ & $512(22)$ & $504(33):$ \\
\hline \multicolumn{10}{|c|}{ Weekend time $\mathrm{min} / \mathrm{d}$} \\
\hline $\begin{array}{l}\text { Sedentary } \\
\text { behaviours }\end{array}$ & $380(130)$ & $454(129)$ & $325(153)^{*}$ & $(378(150)$ & $407(201)$ & $374(138)$ & $383(111)$ & $480(205)$ & $402(175)$ \\
\hline Sleep & $613(56)$ & $607(90)$ & $656(58)$ & $605(83)$ & $636(67)$ & $618(92)$ & $595(95)$. & $596(88)$. & $642(78)=$ \\
\hline $\begin{array}{l}\text { * denotes a } \\
\text { ** denotes } \\
* * * \text { denote }\end{array}$ & $\begin{array}{l}\text { nificant e } \\
\text { gnificant } \\
\text { significan }\end{array}$ & $\begin{array}{l}\text { f age (pl } \\
\text { of age ( }\end{array}$ & $\begin{array}{l}<.05) \\
(p<.01) \\
(p \leq .001)\end{array}$ & tonon-parame & etric analysis (F & dman Test) & $\begin{array}{l}\text { Cohort } \mathrm{A}= \\
\text { Cohort }= \\
\text { Cohort } \mathrm{E}=\end{array}$ & $\begin{array}{l}6 \text { at baselin } \\
8 \text { at baselin } \\
10 \text { at basel }\end{array}$ & \\
\hline
\end{tabular}


Table 8.2. Girls' descriptive data for longitudinal analysis. Values are means (standard deviations).

\begin{tabular}{|c|c|c|c|c|c|c|c|c|c|}
\hline \multirow[b]{3}{*}{ Age } & \multicolumn{3}{|c|}{ Cohort A $(\mathrm{N}=14)$} & \multicolumn{3}{|c|}{ 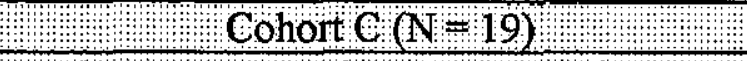 } & \multicolumn{3}{|c|}{ Cohort E $(N=7)$} \\
\hline & Phase 1 & Phase 2 & Phase 3 & Phase 1 & Phase 2 & Phase 3 & Phase 1. & Phase 2 , & Phase 3 \\
\hline & $11.0(0.3)$ & $11.5(0.3)$ & $11.9(0.3)$ & $132(03)$ & $138(0,3)$ & $143(03)$ & $14.9(02)$ & $15.4(02)$ & $15.8(0.2)$ \\
\hline BMI & $17.4(1.8)$ & $17.5(2.1)$ & $17.8(2.0)^{*}$ & $198(3.6)$ & $207(40)$ & $(4.5)^{* * * * 1}$ & $19.6(1 / 8)$ & $20.0(2.2)$ & $20.1(2,1)$ \\
\hline SKF \% & $19.7(4.7)$ & $20.2(4.8)$ & $19.7(4.3)$ & $205(58)$ & $222(65)$ & $23.4(6.6)^{* * *}$ & $193(2.8)$ & $20.6(3.4)$ & $20.4(3.0)$ \\
\hline $\begin{array}{l}\text { Waist-Hip } \\
\text { Ratio }\end{array}$ & $.791(.023)$ & $.782(.025)$ & $.787(.027)$ & $756(039)$ & $751(040)$ & $739(033) * *$ & $722(.032)$ & $728(030)$ & $724(025)$ \\
\hline $\begin{array}{l}\text { Body } \\
\text { Image }\end{array}$ & $2.8(0.6)$ & $2.6(0.5)$ & $2.8(0.4)$ & $25(08)$ & $24(0.6)$ & $2.4(0.7)$ & $2.5(0.7)$ & $2,5(08)$ & $2.4(0.7)$ \\
\hline \multicolumn{10}{|c|}{ Weekday time $\mathrm{min} / \mathrm{d}$} \\
\hline $\begin{array}{l}\text { Sedentary } \\
\text { behaviours }\end{array}$ & $192(73)$ & $220(48)$ & $213(51)$ & $228(65)$ & $232(75)$ & $258(82)$ & $294(76)$ & $251(59)$ & $275(73)$. \\
\hline Sleep & $591(57)$ & $565(35)$ & $550(29)^{* * \dagger}$ & $546(34)$ & $529(37)$ & $527(45)$ & $503(32)$ & $508(21) \div$ & $523(19):$ \\
\hline \multicolumn{10}{|c|}{ Weekend time $\mathrm{min} / \mathrm{d}$} \\
\hline $\begin{array}{l}\text { Sedentary } \\
\text { behaviours }\end{array}$ & $337(119)$ & $357(129)$ & $302(100)$ & $335(149)$ & $343(159)$ & $406(175)$ & $370(63)$ & $248(159)$ & $370(164)$ \\
\hline Sleep & $643(102)$ & $680(69)$ & $681(100)$ & $634(101)$ & $644(101)$ & $623(103)$ & $638(63)$ & $600(39)$ & $573(87)$ \\
\hline \multicolumn{4}{|c|}{$\begin{array}{l}{ }^{*} \text { denotes a significant effect of age (phase) }(p<.05) \\
* * \text { denotes a significant effect of age (phase) }(p<.01) \\
* * * \text { denotes a significant effect of age (phase) }(p \leq .001)\end{array}$} & t nonn-parar & analysis ( & dman Test) & \multicolumn{3}{|c|}{$\begin{array}{l}\text { Cohort } A=\text { Year } 6 \text { at baseline. } \\
\text { Cohort C = Y ear } 8 \text { at baseline. } \\
\text { Cohort } \mathrm{B}=\text { = Year } 10 \text { at baseline. }\end{array}$} \\
\hline
\end{tabular}




\section{Analysis of Covariance}

The primary aim of the longitudinal study was to assess whether changing pubertal status had any effect on time spent in free-time sedentary behaviours. To test for any such effect, the change in total sedentary time was compared between participants who changed pubertal stage and those who did not. Phase 1 sedentary time and change in total sleep time (log-transformed change in total sleep time for boys' weekday data) were entered as covariates in order to control for the effect of these two variables. The results can be seen in Table 8.3 .

The only significant results were found for boys' weekday behaviours. The increase in sedentary time for those who changed pubertal group was significant in comparison to those who stayed at the same level, for whom total sedentary time was reduced. It should be noted that there was a minor violation of the assumption of homogeneity of regression for girls' weekend data.

Table 8.3. Adjusted mean (standard error) of change in time, in minutes per day, spent in sedentary behaviours with Phase 1 sedentary time and change in sleep time as covariates.

\begin{tabular}{llllll}
\hline & \multicolumn{2}{c}{ Boys } & & \multicolumn{2}{c}{ Girls } \\
\cline { 2 - 3 } \cline { 5 - 6 } & $\begin{array}{c}\text { No pubertal } \\
\text { change }\end{array}$ & $\begin{array}{c}\text { Pubertal } \\
\text { change }\end{array}$ & & $\begin{array}{c}\text { No pubertal } \\
\text { change }\end{array}$ & $\begin{array}{c}\text { Pubertal } \\
\text { change }\end{array}$ \\
\hline $\begin{array}{l}\text { Weekday } \\
\begin{array}{l}\text { Sedentary time } \\
\text { Weekend }\end{array}\end{array}$ & $-22(13)$ & $48(16)^{* *}$ & & $10(11)$ & $21(20)$ \\
Sedentary time & $-32(26)$ & $25(33)$ & $20(27)$ & $2(45)$ \\
\hline
\end{tabular}

$* * \mathrm{p}<.01$

\section{Correlations}

A secondary aim of the longitudinal study was to examine whether associations between changing body composition and changing body image during puberty could be used to explain any changes in sedentary time. Correlations between body compositional changes and change in body image can be seen in Tables 8.4 and 8.5 . 
Correlations between different measures of body composition were strongly positive, particularly for participants who increased their pubertal stage, but no significant relations between changing body composition and body image were found for boys or girls $(p>.05)$. These results suggest that changing body composition has no significant relationship with changing body image.

Table 8.4. Correlations between changes of body composition and body image for participants who did not change pubertal stage.

\begin{tabular}{llll}
\hline Boys $(\mathrm{n}=26)$ & $\begin{array}{l}\text { Change in BMI } \\
\text { centile }\end{array}$ & $\begin{array}{l}\text { Change in \% } \\
\text { Body Fat }\end{array}$ & $\begin{array}{l}\text { Change in Body } \\
\text { Image }\end{array}$ \\
\hline Change in BMI $\dagger$ & $.91^{* * *}$ & .27 & .20 \\
Change in BMI centile & & .33 & .16 \\
Change in \% Body Fat $\dagger$ & & & .26 \\
Change in Waist-Hip Ratio & & & .10
\end{tabular}

\begin{tabular}{llll}
\hline Girls $(\mathrm{n}=29)$ & $\begin{array}{l}\text { Change in BMI } \\
\text { centile }\end{array}$ & $\begin{array}{l}\text { Change in \% } \\
\text { Body Fat }\end{array}$ & $\begin{array}{l}\text { Change in Body } \\
\text { Image }\end{array}$ \\
\hline Change in BMI $\dagger$ & $.91^{* * *}$ & $.71^{* * *}$ & -.05 \\
Change in BMI centile & & $.66^{* * *}$ & -.11 \\
Change in \% Body Fat $\dagger$ & & -.10 \\
Change in Waist-Hip Ratio & & -.04 \\
\hline$* * * \mathrm{p}<.001$ (1-tailed) & & \\
$\dagger$ correlations calculated using Spearman's Rho correlation coefficient.
\end{tabular}

Table 8.5. Correlations between changes of body composition and body image for participants who increased puberty by one stage.

\begin{tabular}{llll}
\hline Boys $(\mathrm{n}=17)$ & $\begin{array}{l}\text { Change in BMI } \\
\text { centile }\end{array}$ & $\begin{array}{l}\text { Change in \% } \\
\text { Body Fat }\end{array}$ & $\begin{array}{l}\text { Change in } \\
\text { Body Image }\end{array}$ \\
\hline Change in BMI $\dagger$ & $.95^{* * *}$ & $.70^{* * *}$ & -.01 \\
Change in BMI centile & & $.81^{* * *}$ & .00 \\
Change in \% Body Fat & & & -.00 \\
Change in Waist-Hip Ratio & & & -.26 \\
\hline Girls (n = 11) & Change in BMI & Change in \% & Change in Body \\
& centile & Body Fat & Image \\
\hline Change in BMI $\dagger$ & $.99^{* * *}$ & $.95^{* * *}$ & .04 \\
Change in BMI centile $\dagger$ & & $.96^{* * *}$ & .01 \\
Change in \% Body Fat & & & .11 \\
Change in Waist-Hip Ratio & & & .26 \\
\hline
\end{tabular}

$* * * \mathrm{p}<.001$ (1-tailed)

$\dagger$ correlations calculated using Spearman's Rho correlation coefficient. 
Table 8.6 shows biserial correlations between the change in pubertal status (discrete dichotomous dependant variable: no change in pubertal stage versus increased puberty by one stage) and change in body composition and body image. No significant relationships were found for either gender $(p>.05)$.

Table 8.6. Biserial correlations between change in pubertal status, changes of body composition, and changes of body image.

\begin{tabular}{|c|c|c|c|c|c|}
\hline Boys $(n=43)$ & $\begin{array}{l}\text { Change } \\
\text { in BMI }\end{array}$ & $\begin{array}{l}\text { Change in } \\
\text { BMI centile }\end{array}$ & $\begin{array}{l}\text { Change in } \\
\% \text { Body Fat }\end{array}$ & $\begin{array}{l}\text { Change in } \\
\text { Waist-Hip Ratio }\end{array}$ & $\begin{array}{l}\text { Change in } \\
\text { Body Image }\end{array}$ \\
\hline $\begin{array}{l}\text { Change in } \\
\text { Pubertal } \\
\text { status }\end{array}$ & .00 & .31 & -.10 & .13 & .15 \\
\hline Girls $(n=40)$ & $\begin{array}{l}\text { Change } \\
\text { in BMI }\end{array}$ & $\begin{array}{l}\text { Change in } \\
\text { BMI centile }\end{array}$ & $\begin{array}{l}\text { Change in } \\
\% \text { Body Fat }\end{array}$ & $\begin{array}{l}\text { Change in } \\
\text { Waist-Hip Ratio }\end{array}$ & $\begin{array}{l}\text { Change in } \\
\text { Body Image }\end{array}$ \\
\hline $\begin{array}{l}\text { Change in } \\
\text { Pubertal } \\
\text { status }\end{array}$ & -.22 & -.01 & -.17 & -.03 & -.22 \\
\hline
\end{tabular}

These results were confirmed by comparison of means between those who changed pubertal status and those who did not (independent samples $t$-test for normal data, Mann-Whitney U-test for skewed data). No significant differences were seen for measures of change in body composition or change in body image. Controlling for Phase 1 pubertal status did not affect the significance levels ( $p>.05$ in each case). These results suggest that pubertal changes have no relationship with body compositional changes. This finding is in contrast to well-established knowledge (Rogol et al., 2002) and is discussed in the next chapter.

\section{Multiple Regression Analysis}

Multiple regression analysis using a hierarchical (blockwise) entry method was used to examine whether any other variables had a direct effect on change in sedentary behaviour. In addition to change in pubertal status, the effects of changing BMI, percentage body fat, waist-hip ratio and body image were individually tested (one at a time). 


\section{Weekday}

In the boys' model, Phase 1 sedentary behaviour and log-transformed change in total sleep time were entered first in order to control for the effect of these variables. The effect of adding change in pubertal stage into the model was to explain a significant increase of $14 \%$ of the variance $\left(\mathrm{F}_{1,39}=10.5, \mathrm{p}=.002\right)$ with increasing pubertal status being associated with an increase in sedentary behaviour. This result confirms the findings of the ANCOVA as expected because multiple regression is an extension of analysis of variance. Body composition and body image variables were not found to significantly increase the variance.

In the girls' model, after controlling for the effects of Phase 1 sedentary behaviour and change in sleep time, change in pubertal stage was not found to be a significant predictor. However, when change in physical attractiveness was entered, a significant increment of $10 \%$ of the variance was explained $\left(\mathrm{F}_{1,35}=4.9, \mathrm{p}=.034\right)$ with a reduction in body image being associated with an increase in sedentary behaviour. Body composition variables were not found to significantly increase the variance.

\section{Weekend Day}

In the boys' model, after controlling for the effects of Phase 1 weekend sedentary time and change in weekend sleep, no other variables were found to be significant predictors of the change in weekend sedentary time.

In the girls' model, however, after controlling for the effects of Phase 1 weekend sedentary time and change in weekend sleep, change in log-transformed percentage body fat was able to explain an increase of $9 \%$ of the variance $\left(F_{1,32}=4.9, p=.034\right)$ with an increase in body fat being associated with an increase in weekend sedentary behaviour. No other variables were found to be significant predictors. 


\section{Chapter 9: Longitudinal Discussion}

Randomised, controlled studies have shown that reducing the time spent in sedentary behaviours may help to reduce obesity among young people aged $8-12$ years. Gortmaker et al. (1999) found that, for a group of 11 to 12-year-old students, the prevalence of obesity among girls who had undertaken an intervention was reduced compared with controls although no differences were found for boys. In this study the intervention involved a 2-year school-based interdisciplinary intervention including decreasing television viewing and consumption of high-fat foods, and increasing moderate and vigorous physical activity. Regression analysis indicated that only the reduction in television viewing could explain the intervention effect. In addition, Epstein, Paluch, Gordy, and Dorn (2000) reported that, for 76 obese 8 to 12-year-old children, a 2-year intervention of either decreased sedentary behaviour or increased physical activity was associated with significant decreases in percent overweight and body fat. It was also reported that reducing time in targeted sedentary behaviours resulted in a redistribution of time to other non-targeted sedentary behaviours as well as physically active behaviours. However, outside of such controlled trials it is important to identify the determinants of such behaviours in free-living individuals in order to design 'real world' interventions. The aim of the current longitudinal study was to determine the effect of pubertal changes in body composition and body image on any within-participant changes in free-time sedentary behaviour over a 12-month period during adolescence.

\section{Repeated Measures}

The pattern of change in boys' BMI with advancing age was reflective of the pattern shown by nationally representative data (Cole et al., 1995) as BMI increased steadily with age in each of the cohorts. The pattern of change for girls was more equivocal with an effect of age seen in Cohorts $\mathrm{A}$ and $\mathrm{C}$ but not in Cohort $\mathrm{E}$. This may be indicative of the older girls having reached the end of pubertal changes whilst the boys were still continuing with puberty. Alternatively, this lack of a significant finding for older girls may be due to weak statistical power caused by low participant 
numbers in this cohort compared with the recommendations of the power analysis (see Chapter 4).

Changes to percentage body fat followed the expected pattern during normal growth at puberty (Rogol et al., 2002) particularly in Cohort C. Boys' percentage body fat decreased as they developed bodies that were more muscular whereas girls' percentage body fat increased. The waist-hip ratio showed a significant drop between Phases 1 and 3 for Cohort $\mathrm{C}$ girls as they increased the amount of gluteo-femoral fat. The most significant changes may have occurred in Cohort $\mathrm{C}$ because this is the age range that would be expected to experience the greatest pubertal development. For boys the maximal loss of fat in the upper limbs occurs at the time of peak height velocity (Rogol et al., 2002), which is approximately 14 years of age (Marshall \& Tanner, 1970). In girls, however, peak height velocity is achieved at approximately 12 years of age (Marshall \& Tanner, 1969) after which fat accumulation resumes at a rate of approximately $1.14 \mathrm{~kg} /$ year, increasing both the fat mass and fat percentage (Rogol et al., 2002).

Some caution must be exercised when using the results of non-parametric techniques because many such tests are based on rankings of the original scores rather than their real values. For example, the Friedman Test works by comparing the differences between mean ranks of scores without taking the absolute differences between scores into account. One particular problem occurs with increasing numbers of tied ranks leading to inaccuracies (Howitt \& Cramer, 2000). In addition, use of non-parametric statistics increases the chance of a type II error as they have less power than equivalent parametric tests (Field, 2000). However, in each case here the levels of significance from non-parametric tests were similar to those achieved when repeated measures ANOVAs were substituted in place of the Friedman Tests.

No evidence was found for a relationship between age (phase) and time spent in weekday sedentary behaviours, a result that is supported by previous research (Lawrence et al., 1986), (Shann, 2001), (Wolf et al., 1993). Other researchers have found contrasting evidence however. For example, Schmitz et al. (2002) reported that, for girls, age was a demographic factor predictive of sedentary leisure habits with older girls reporting lower levels of sedentary behaviour. A result contrary to the 
findings of Deheeger et al. (2002) and Bradley et al: (2000) who both found sedentary behaviour to increase with age. This finding is also in contrast to the results of Phase 1 of the current study (before sleep was controlled for). Although the reasons for equivocal findings in earlier research have already been discussed in terms of deficient measuring tools, one other reason for a lack of relationship within the current data may have been that assessments only six months apart may not allow time for age-based changes to occur that are big enough to be detected by the diary.

The significant decrease in Cohort A boys' weekend sedentary behaviour between Phases 2 and 3 may have been due to the higher assessment of temperature in Phase 3 by this group, increasing the opportunities for alternative activities. The temperature of Phase 1 was rated as between those of Phases 2 and 3 but no significant differences in sedentary behaviour were apparent. However, the smaller temperature differences may not have been enough to affect any behavioural changes. No significant differences in sedentary behaviour were found for Cohort A girls although Phase 2 was rated as significantly cooler. This suggests that different triggers of weekend behaviour may be present between the genders.

Differences in the rating of temperatures during the weekdays were not associated with any concurrent changes in sedentary behaviour time. This may be because during weekdays, when there is less opportunity after school to spend time outdoors compared with the weekend, behaviours are less affected by ecological factors such as the temperature.

\section{Analysis of Covariance}

The primary purpose of this study was to test the effect of changes in pubertal status on the total time spent in free-time sedentary behaviours. A further aim was to examine the effects of, and interactions between, changing body composition, as measured by percentage body fat, BMI or waist-hip ratio, and changing body image as measured by self-assessed physical attractiveness. After controlling for the effects of Phase 1 sedentary behaviour and change in total sleep time, a significant difference in change in weekday sedentary behaviour between participants who changed pubertal status and those who did not was found for boys but not for girls. This finding 
suggests that differences in determinants of sedentary behaviour may be present between the genders and is reflective of the results of studies that have found gender differences in correlates of active behaviours (Aaron et al., 1993; Biddle \& Armstrong, 1992; Schmitz et al., 2002).

In terms of change in weekend sedentary behaviour, no differences were found between pubertal change groups for either boys or girls. This finding may reflect the fact that a single day of weekend recording is not sufficient to record habitual behaviour. Alternatively, correlates of weekend behaviour may be unrelated to pubertal status but related to other factors, a theory that is supported by the multiple regression analysis.

The first part of the original hypothesis that, 'increases in total time spent in sedentary behaviours will be greater in participants who increase their pubertal status' was, therefore, only partly supported by the data.

\section{Correlations}

Biserial correlations between the change in pubertal status and change in body composition or change in body image did not follow the hypothesised patterns. It was expected that those who changed pubertal status would show a greater change in percentage body fat, BMI, and BMI centile. However, no significant correlations were found between the dependent variable and any of the independent variables. Additionally, comparison between those who changed pubertal stage versus those who did not change found no significant differences between the means of the body compositional variables. The reason for this unexpected finding is unclear. One possible explanation may have been the differences in absolute pubertal status between participants. As described above, changes in body composition vary according to stage of pubertal development and gender (Marshall \& Tanner, 1969, 1970; Rogol et al., 2002). However, controlling for Phase 1 pubertal status did not affect the analysis. It may be the case that the changes in body composition within this period were not large enough to be statistically significant for such a small sample. Alternatively, the method of assessing pubertal stage change may not have been appropriate. 
Correlations between change in body composition and change in body image also failed to confirm the hypothesised negative relationship. For boys this is consistent with some previous research (Barker \& Galambos, 2003; French et al., 1996; Kolody \& Sallis, 1995), although other studies have found BMI to be positively related (McCabe \& Ricciardelli, 2003), and body fat to be negatively related to body image (Duncan, Woodfield, O'Neill, \& Al-Nakeeb, 2002) in boys. However, the current results are inconsistent with previous research in girls, which has found negative relationships between body image and BMI (Barker \& Galambos, 2003; French et al., 1996; Kolody \& Sallis, 1995; Sands \& Wardle, 2003) and skinfolds (Duncan, Woodfield, O'Neill, \& Al-Nakeeb, 2002; Kimm et al., 1997), or conversely a positive relationship between BMI and body image (McCabe \& Ricciardelli, 2003) (see earlier discussion on page 29). In addition, no significant correlations were found between change in fat distribution and body image. This contradicts the ideas of Page and Fox (1997) that increased fat storage in the hips, thighs and buttocks may be a cause of falling body image with increasing age in adolescent girls. This may be because body compositional changes within a 12-month period are not extreme enough to impact on body image. Alternatively, the lack of any significant findings in the current study may, once again, be due to a lack of sensitivity within the measurement methods to detect such small changes within a cohort of this size (i.e., a lack of power).

Taken at face value however, these findings suggest that the significant increase in weekday sedentary behaviour seen for boys who had increased pubertal stage was not due to a change in behavioural choice triggered by reduced body image associated with changing body composition. Thus, the second part of the original hypothesis, that 'body composition and body image will be mediators of [a] difference [in changes in sedentary behaviour] via changes in behavioural choices' was not supported by this data.

\section{Multiple Regression Analysis}

After controlling for the effects of Phase 1 sedentary behaviour and change in total sleep time, the addition of change in pubertal status supported the findings of the ANCOVA and was found to be a significant predictor of the change in weekday 
sedentary behaviour for boys only. However, the multiple regression analysis allowed the exploration of other variables other than puberty that may be directly associated with the change in sedentary behaviour and for girls, but not boys, a change in physical attractiveness (body image) was found to be a significant weekday predictor. This gender difference is supportive of the results found for adults by Williams, Sallis, Calfas, and Burke (1999). In their study, body image dissatisfaction was a significant predictor of television watching in females, but not males, after controlling for ethnicity and employment status.

In the current study, the lack of correlations between change in pubertal status, body composition or fat distribution and change in body image discussed previously suggest that the contribution of physical attractiveness for girls is independent of any changes due to puberty and also independent of changes to body fat. This finding supports part of the hypothesis that sedentary behaviour may be associated with body image. However, this association may not be related to maturity.

During the weekend none of the measured variables were able to explain any of the variance in boys' sedentary time. However, for girls an increase in body fat was associated with an increase in sedentary behaviour. This was not accompanied by any association with body image and it may be the case that the direction of hypothesised association from body composition to sedentary behaviour was reversed. In other words, girls who increased body fat did not choose more sedentary behaviours, but those who were more sedentary increased their body fat to a greater extent. In contrast to the findings from Phase 1 , which were based on weekday data, these results from the weekend do, in part, support the theory of Gortmaker et al (1996) that reductions in television viewing (as a dominant sedentary behaviour) could help to reduce obesity in young people. Additionally, the finding of a gender difference within such a relationship is supported by previous research. Berkey et al. (2003) found that for a large sample of 11,88711 - to 15 -year-olds a one year increase in inactivity (television, videos, and video game playing) was associated with increasing BMI in girls but not boys. 


\section{Chapter 10: Conclusions}

\section{Phase 1 (Cross-Sectional)}

Results showed that differences in sedentary behaviour between pubertal groups were partly explained by greater opportunity due to less sleep time. Although girls' body image was associated with body compositional differences in puberty, the relationship with sedentary behaviour was not strong enough to infer behavioural choice differences. For boys, age was more highly correlated with sedentary behaviour than pubertal status. This may be because the pubertal status-body composition-body image relationship that was hypothesised is less defined in boys'. It also suggests that determinants of sedentary behaviour in boys may be more closely linked to differences in age than pubertal status although the greater amount of time seen in Year 10 may have been due to the changing school environment.

Figures 10.1a and $\mathrm{b}$ summarise the measured cross-sectional partial correlations between variables that were hypothesised to exist in Figure 1.1.

\section{Longitudinal}

Findings from the Phase 1 results were used to inform the longitudinal analysis, in particular the need to control for the effect of sleep. Some inconsistencies were found between the cross-sectional results from Phase 1 and those collected longitudinally. Such inconsistencies may be due to the different effects of between-participants differences compared to within-participants changes. To collect evidence in support of the a priori hypothesis, results of the longitudinal analysis are more powerful because there is greater opportunity for cross-sectional, between-participants differences to have been affected by variables external to those being studied.

The hypothesis of the current study was that increases in total time spent in sedentary behaviours would be greater in participants who increase their pubertal status, and that body composition and body image would be mediators of this difference via changes in behavioural choices. The longitudinal results of this study could only support a part 
of this hypothesis, as only boys' weekday changes in sedentary behaviour were associated with pubertal changes. No associations were found between changes in body composition, body image, and sedentary behaviour to support the behavioural choice hypothesis in boys. More evidence for such a theory was available for girls as change in body image was a significant predictor of change in weekday sedentary behaviour. There was, however, no evidence to indicate that this was associated with increased puberty or change in body composition.

The consequences of the longitudinal results are that no single approach to reducing sedentary behaviour during adolescence is appropriate. Solutions should be gender specific and may vary for weekday and weekend day behaviour. 
Figure 10.1a Measured partial correlations between sedentary behaviours and possible determinants (boys).

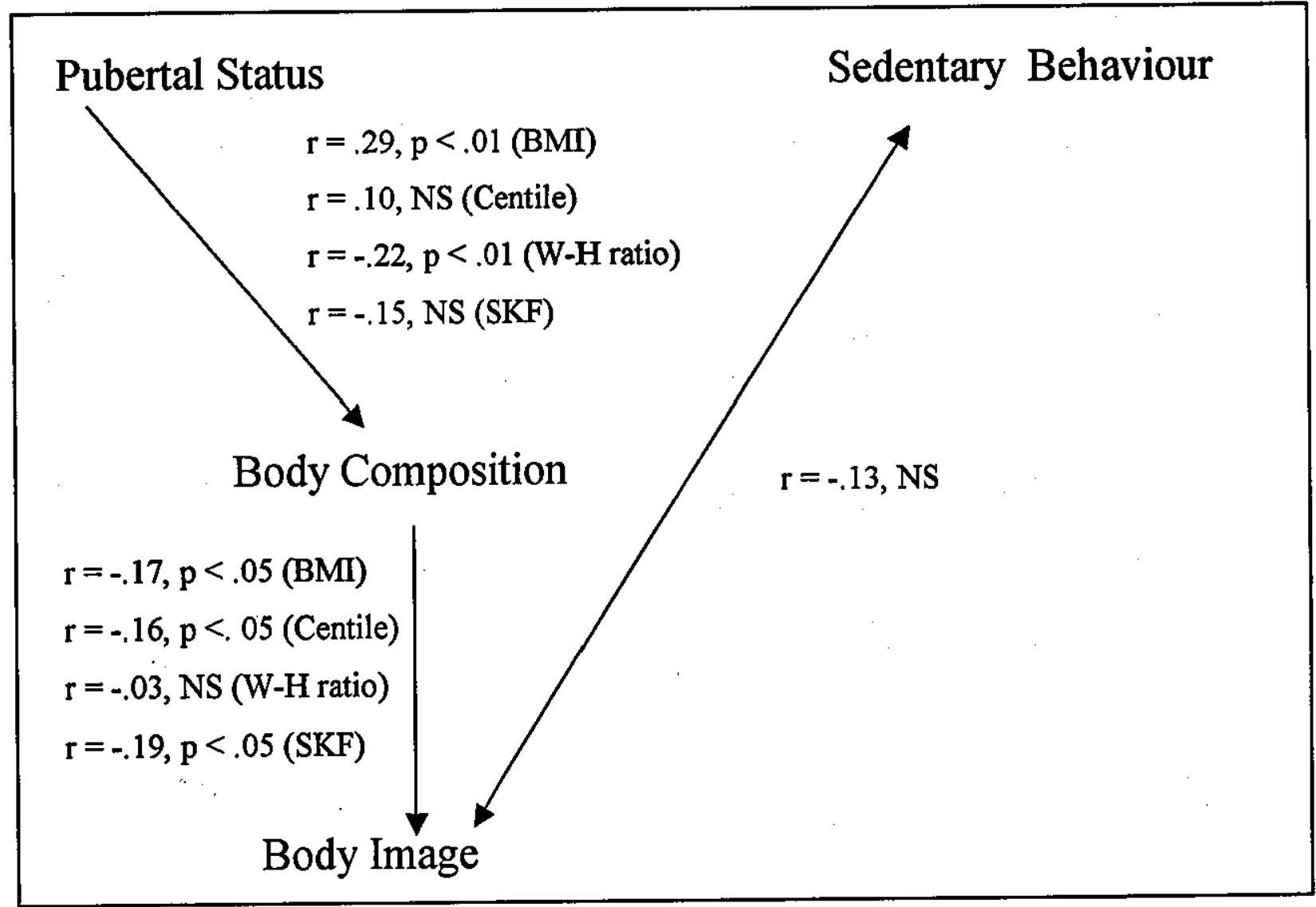

Figure 10.1b Measured partial correlations between sedentary behaviours and possible determinants (girls).

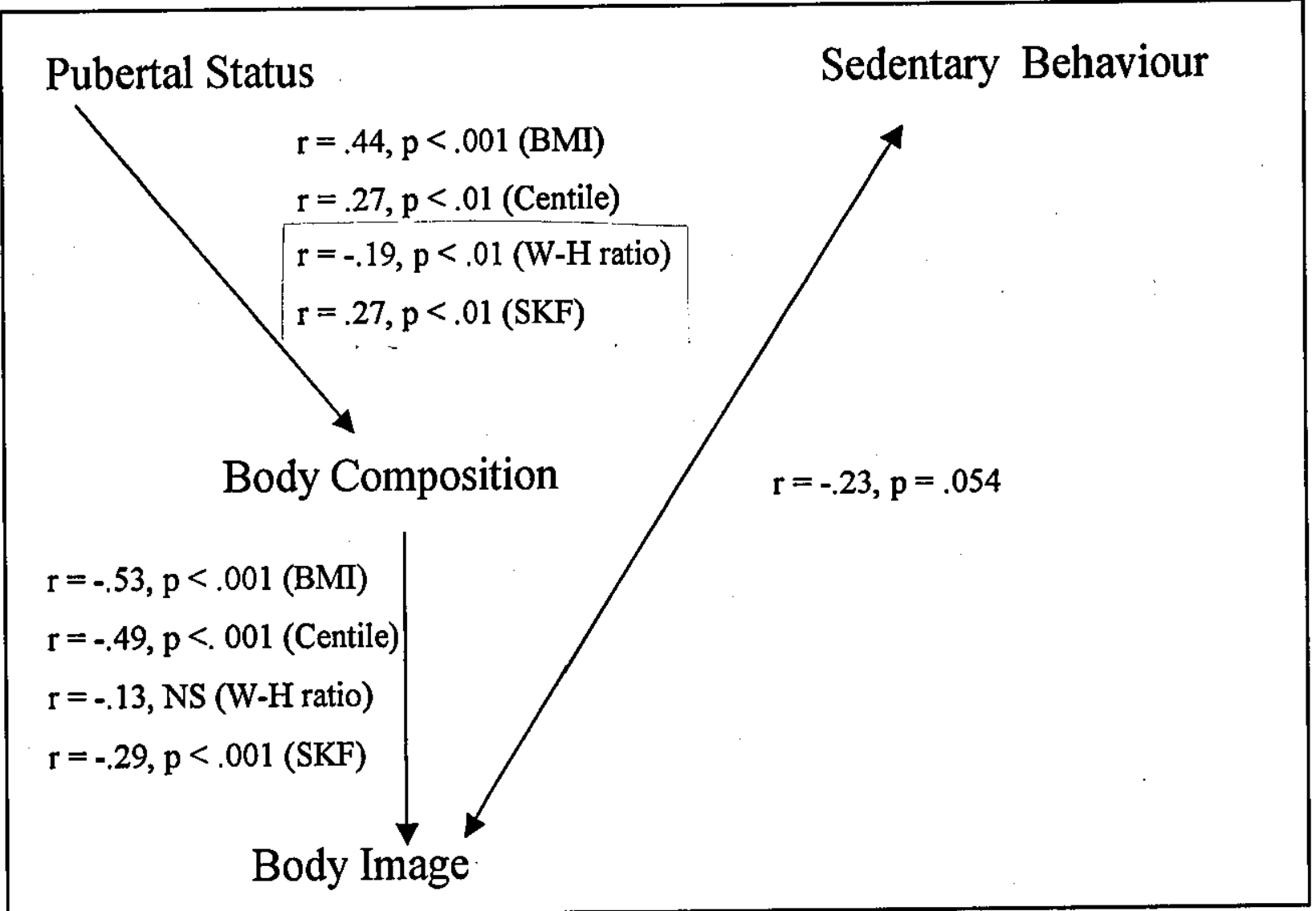




\section{Future Research Directions}

A good starting point for future research directions is to identify limitations present within the current study. Firstly, final participant numbers failed to reach the level required by the power analysis. Therefore, a priority of future research is to try to reproduce these results in a similar sample with greater participant numbers in order to demonstrate that results were not affected by any loss of power. It would also be advantageous to extend the participant base to include a more ethnically diverse cohort because no non-white participants were present in the final analysis.

This study also highlights the need to develop a better measure of pubertal development in boys. The reduction of pubertal groups from four to three was an unwelcome development in latter stages of the research. A non-invasive method of identifying the transition from late-pubertal to adult is essential.

Any future research using the diary based momentary time sampling approach would benefit from using a greater number of behaviour categories, in particular active behaviours. Analysis of time spent in sporting behaviours from the current data set may be impeded by the overly comprehensive group of behaviours that were coded into the category of sport and exercise e.g. dog walking.

Aside from identifying weaknesses within the study the findings of this thesis may also be used to identify possible future directions. Results from Gortmaker et al (1996) in conjunction with those from Phase 1 of this study (see Phase 1 discussion) suggested that television more than total sedentary time might be affected by other obesogenic factors such as snacking. This emphasises the need for this field of research to adopt a more holistic approach that includes factors from both sides of the energy balance equation, that is a comprehensive measure of dietary intake in addition to behaviours.

Results from the multiple regressions explained only $0-14 \%$ of the variance in sedentary behaviour change over time. This means that a great deal of the variance remains to be explained, in particular for boys during the weekend. There is therefore 
obvious scope for investigating other variables that may be responsible for these changes.

To be able to use these results to help design intervention studies, the collection of qualitative data from a subset of participants would be useful in order to ascertain why some of the identified 'relationships were present. This would involve in-depth individual interviews focussing on the salient factors arising from this thesis.

Finally, there is a need for longitudinal projects that study the effects of changes in sedentary behaviour, body composition, and body image over time in age groups other than those studied in this thesis. 


\section{$\underline{\underline{\text { References }}}$}

Aaron, D. J., Kriska, A. M., Dearwater, S. R., Anderson, R. L., Olsen, T. L., Cauley, J. A., et al. (1993). The Epidemiology of Leisure Physical-Activity in an Adolescent Population. Medicine and Science in Sports and Exercise, 25(7), 847-853.

Ainsworth, B. E., Haskell, W. L., Leon, A. S., Jacobs, D. R., Jr., Montoye, H. J., Sallis, J. F., et al. (1993). Compendium of physical activities: classification of energy costs of human physical activities. Medicine and Science in Sports and Exercise, 25(1), 71-80.

Ainsworth, B. E., Haskell, W. L., Whitt, M. C., Irwin, M. L., Swartz, A. M., Strath, S. J., et al. (2000). Compendium of physical activities: an update of activity codes and MET intensities. Medicine and Science in Sports and Exercise, 32(9 Suppl), S498-504.

American Academy of Pediatrics, Committee on Communications. (1995). Children, adolescents, and television. Pediatrics, 96(4 Pt 1), 786-787.

Armstrong, N., Welsman, J. R., \& Kirby, B. J. (2000). Longitudinal changes in 11-13-year-olds' physical activity. Acta Paediatrica, 89(7), 775-780.

Barker, E. T., \& Galambos, N. L. (2003). Body dissatisfaction of adolescent girls and boys: Risk and resource factors. Journal of Early Adolescence, 23(2), 141165 .

Baumgartner, R. N., Chumlea, W. C., \& Roche, A. F. (1989). Estimation of Body-Composition from Bioelectric Impedance of Body Segments. American Journal of Clinical Nutrition, 50(2), 221-226.

Baumgartner, R. N., Ross, R., \& Heymsfield, S. B. (1998). Does adipose tissue influence bioelectric impedance in obese men and women? Journal of Applied Physiology, 84(1), 257-262.

Behnke, A. R. (1959). The Estimation of Lean Body Weight From "Skeletal" Measurements. Human Biology, 31(4), 295-315.

Bell, W. (1993). Body size and shape: a longitudinal investigation of active and sedentary boys during adolescence. Journal of Sports Sciences, 11(2), 127-138. 
Bellizzi, M. C., \& Dietz, W. H. (1999). Workshop on childhood obesity:summary of the discussion. American Journal of Clinical Nutrition, 70(1), 173S-175S.

Berkey, C. S., Rockett, H. R., Field, A. E., Gillman, M. W., Frazier, A. L., Camargo, C. A., Jr., et al. (2000). Activity, dietary intake, and weight changes in a longitudinal study of preadolescent and adolescent boys and girls. Pediatrics, 105(4), E56.

Berkey, C. S., Rockett, H. R. H., Gillman, M. W., \& Colditz, G. A. (2003). One-year changes in activity and in inactivity among 10-to 15- year-old boys and girls: Relationship to change in body mass index. Pediatrics, 111(4), 836-843.

Bernstein, M. S., Morabia, A., \& Sloutskis, D. (1999). Definition and prevalence of sedentarism in an urban population. American Journal of Public Health, 89(6), 862-867.

Beunen, G. P., Malina, R. M., Lefevre, J., Claessens, A. L., Renson, R., \& Simons, J. (1997). Prediction of adult stature and noninvasive assessment of biological maturation. Medicine and Science in Sports and Exercise, 29(2), 225-230.

Beunen, G. P., Malina, R. M., Renson, R., Simons, J., Ostyn, M., \& Lefevre, J. (1992). Physical activity and growth maturation and performance: a longitudinal study. Medicine and Science in Sports and Exercise, 24(5), 576-585.

Biddle, S., \& Armstrong, N. (1992). Children's physical activity: an exploratory study of psychological correlates. Social Science in Medicine, 34(3), 325331.

Biddle, S., Cavill, N., \& Sallis, J. F. (1998). Policy framework for young people and health-enhancing physical activity. In S. Biddle \& N. Cavill \& J. F. Sallis (Eds.), Young and Active. London: Health Education Authority.

Billewicz, W. Z., Fellowes, H. M., \& Thomson, A. M. (1981). Pubertal changes in boys and girls in Newcastle upon Tyne. Annals of Human Biology, 8(3), 211-219.

Bindra, D., \& Blond, J. (1958). A time-sample method for measuring general activity and its components. Canadian Journal of Psychology, 12, 74-76.

Bouten, C. V., Verboeket-van de Venne, W. P., Westerterp, K. R., Verduin, M., \& Janssen, J. D. (1996). Daily physical activity assessment: comparison between movement registration and doubly labeled water. Journal of Applied Physiology, 81(2), 1019-1026. 
Bradley, C. B., McMurray, R. G., Harrell, J. S., \& Deng, S. B. (2000). Changes in common activities of 3rd through 10th graders: the CHIC Study. Medicine and Science in Sports and Exercise, 32(12), 2071-2078.

Brener, N. D., McManus, T., Galuska, D. A., Lowry, R., \& Wechsler, H. (2003). Reliability and validity of self-reported height and weight among high school students. Journal of Adolescent Health, 32(4), 281-287.

Brooks-Gunn, J., \& Warren, M. P. (1988). The Psychological Significance of Secondary Sexual Characteristics in Nine- to Eleven-Year-Old Girls. Child Development, 59, 1061-1069.

Brooks-Gunn, J., Warren, M. P., Rosso, J., \& Gargiulo, J. (1987). Validity of self-report measures of girls' pubertal status. Child Development, 58(3), 829-841.

Brulle, A. R., \& Repp, A. C. (1984). An Investigation of the Accuracy of Momentary Time Sampling Procedures with Time-Series Data. British Journal of Psychology, 75(NOV), 481-485.

Cameron, A. J., Welborn, T. A., Zimmet, P. Z., Dunstan, D. W., Owen, N., Salmon, J., et al. (2003). Overweight and obesity in Australia: the 1999-2000 Australian Diabetes, Obesity and Lifestyle Study (AusDiab). Medical Journal of Australia, 178(9), 427-432.

Cameron, N. (1984). The Measurement of Human Growth. London \& Sydney: Croom Helm.

Carter, J. E. L., \& Heath, B. H. (1990). Somatotyping: Development and Applications (1 ed.). Cambridge: Cambridge University Press.

Cohn, L. D., Adler, N. E., Irwin, C. E., Jr., Millstein, S. G., Kegeles, S. M., \& Stone, G. (1987). Body-figure preferences in male and female adolescents. Journal of Abnormal Psychology, 96(3), 276-279.

Cole, T. J., Bellizzi, M. C., Flegal, K. M., \& Dietz, W. H. (2000). Establishing a standard definition for child overweight and obesity worldwide: international survey. British Medical Journal, 320, 1240-1243.

Cole, T. J., Freeman, J. V., \& Preece, M. A. (1995). Body mass index reference curves for the UK, 1990. Archives of Disease in Children, 73(1), 25-29.

Crawley, H. F., \& Portides, G. (1995). Self-reported versus measured height, weight and body mass index amongst 16-17 year old British teenagers. International Journal of Obesity and Related Metabolic Disorders, 19(8), 579-584. 
Deheeger, M., Bellisle, F., \& Rolland Cachera, M. F. (2002). The French longitudinal study of growth and nutrition: Data in adolescent males and females. Journal of Human Nutrition and Dietetics, 15(6), 429-438.

Dietz, W. H. (1996). The role of lifestyle in health: the epidemiology and consequences of inactivity. The Proceedings of the Nutrition Society, 55(3), 829-840.

Dorn, L. D., Susman, E. J., \& Ponirakis, A. (2003). Pubertal timing and adolescent adjustment and behavior: Conclusions vary by rater. Journal of Youth and Adolescence, 32(3), 157-167.

Dubas, J. S., Graber, J. A., \& Petersen, A. C. (1991). A longitudinal investigation of adolescents' changing perceptions of pubertal timing. Developmental Psychology, 27(4), 580-586.

Ducharme, J. R., Forest, M. G., De Peretti, E., Sempe, M., Collu, R., \& Bertrand, J. (1976). Plasma adrenal and gonadal sex steroids in human pubertal development. The Journal of Clinical Endocrinology and Metabolism, 42(3), 468-476.

Duke, P. M., Litt, I. F., \& Gross, R. T. (1980). Adolescents' self-assessment of sexual maturation. Pediatrics, 66(6), 918-920.

Duncan, M. J., Woodfield, L. A., O'Neill, S. J., \& Al-Nakeeb, Y. (2002). Relationship between body image and percent body fat among British school children. Perceptual and Motor Skills, 94(1), 197-203.

Duncan, M. J., Woodfield, L. A., O'Neill, S. J., Al-Nakeeb, Y., Nevill, A. M., \& Lane, A. M. (2002). Test-retest stability of body-image scores in a sample of 12-to 14-yr.-olds. Perceptual and Motor Skills, 95(3), 1007-1012.

Epstein, L. H. (1998). Integrating theoretical approaches to promote physical activity. American Journal of Preventive Medicine, 15(4), 257-265.

Epstein, L. H., Paluch, R. A., Cordy, C. C., \& Dorn, J. (2000). Decreasing Sedentary Behaviors in Treating Pediatric Obesity. Archives of Pediatric and Adolescent Medicine, 154, 220-226.

Farthing, M. J., Mattei, A. M., Edwards, C. R., \& Dawson, A. M. (1982). Relationship between plasma testosterone and dihydrotestosterone concentrations and male facial hair growth. The British Journal of Dermatology, 107(5), 559-564.

Feldman, D. E., Barnett, T., Shrier, I., Rossignol, M., \& Abenhaim, L. (2003). Is physical activity differentially associated with different types of sedentary pursuits? Archives of Pediatric and Adolescent Medicine, 157(8), 797-802. 
Field, A. (2000). Discovering Statistics using SPSS for Windows: Advanced Techniques for the Beginner. London: Sage.

Fox, K. R. (1997). The Physical Self and Processes in Self-Esteem Development. In K. R. Fox (Ed.), The Physical Self: from motivation to well-being (pp. 111-139). Champaign, Illinois: Human Kinetics.

Fox, K. R., \& Corbin, C. B. (1989). The Physical Self-Perception Profile: Development and preliminary validation. Journal of Sport and Exercise Psychology, $11(4), 408-430$.

Frankowski, B., Dukeduncan, P., Guillot, A., McDougal, D., Wasserman, R., \& Young, P. (1987a). Young Adolescents Self-Assessment of Sexual-Maturation. American Journal of Diseases of Children, 141(4), 385-386.

Frankowski, B., Dukeduncan, P., Guillot, A., McDougal, D., Wasserman, R., \& Young, P. (1987b). Young Adolescents Self-Assessment of Sexual-Maturation (Abstract). American Journal of Diseases of Children, 141(4), 385-386.

French, S. A., Perry, C. L., Leon, G. R., \& Fulkerson, J. A. (1996). Selfesteem and change in body mass index over 3 years in a cohort of adolescents. Obesity Research, 4(1), 27-33.

Fu, F. H., \& Hao, X. (2002). Physical development and lifestyle of Hong Kong secondary school students. Preventive Medicine, 35(5), 499-505.

Gallagher, M., Walker, K. Z., \& O'Dea, K. (1998). The influence of a breakfast meal on the assessment of body composition using bioelectrical impedance. Eur J Clin Nutr, 52(2), 94-97.

Garrow, J. S. (1982). Treat obesity seriously: a clinical manual. Edinburgh: Churchill Livingstone.

Geiß, H. C., Parhofer, K. G., \& Schwandt, P. (2001). Parameters of childhood obesity and their relationships to cardiovascular risk factors in healthy prepubescent children. International Journal of Obesity, 25, 830-837.

Goodman, E., Hinden, B. R., \& Khandelwal, S. (2000). Accuracy of teen and parental reports of obesity and body mass index. Pediatrics, 106(1 Pt 1), 52-58.

Gordon-Larsen, P. (2001). Obesity-related knowledge, attitudes, and behaviors in obese and non-obese urban Philadelphia female adolescents. Obesity Research, 9(2), 112-118.

Gordon-Larsen, P., McMurray, R. G., \& Popkin, B. M. (2000). Determinants of Adolescent Physical Activity and Inactivity Patterns. Pediatrics, 105(6), e83. 
Gortmaker, S. L., Must, A., Sobol, A. M., Peterson, K., Colditz, G. A., \& Dietz, W. H. (1996). Television viewing as a cause of increasing obesity among children in the United States, 1986-1990. Archives of Pediatrics and Adolescent Medicine, 150(4), 356-362.

Gortmaker, S. L., Peterson, K., Wiecha, J., Sobol, A. M., Dixit, S., Fox, M. K., et al. (1999). Reducing obesity via a school-based interdisciplinary intervention among youth: Planet Health. Archives of Pediatric and Adolescent Medicine, 153(4), 409-418.

Gray, D. S., Bray, G. A., Bauer, M., Kaplan, K., Gemayel, R. W., Greenway, F., et al. (1990). Skinfold thickness measurements in obese subjects. American Journal of Clinical Nutrition, 51, 571-577.

Gray, D. S., Bray, G. A., Gemayel, N., \& Kaplan, K. (1989). Effect of obesity on bioelectrical impedance. American Journal of Clinical Nutrition, 50, 255-260.

Guo, S. S., \& Chumlea, W. C. (1999). Tracking of body mass index in children in relation to overweight in adulthood. American Journal of Clinical Nutrition, 70(1), 145S-148S.

Gupta, D., Attanasio, A., \& Raaf, S. (1975). Plasma estrogen and androgen concentrations in children during adolescence. The Journal of Clinical Endocrinology and Metabolism, 40(4), 636-643.

Hanley, A. J., Harris, S. B., Gittelsohn, J., Wolever, T. M., Saksvig, B., \& Zinman, B. (2000). Overweight among children and adolescents in a Native Canadian community: prevalence and associated factors. American Journal of Clinical Nutrition, 71(3), 693-700.

Harrell, J. S., Gansky, S. A., Bradley, C. B., \& McMurray, R. G. (1997). Leisure time activities of elementary school children. Nursing Research, 46(5), 246253.

Harrop, A., \& Daniels, M. (1985). Momentary Time Sampling with TimeSeries Data - a Commentary. British Journal of Psychology, 76(NOV), 533-537.

Harter, S. (1982). The Perceived Competence Scale for Children. Child Development, 53(1), 87-97.

Harter, S. (1985). Manual for the self-perception profile for children. Denver: University of Denver. 
Harter, S. (1993). Causes and Consequences of Low Self-Esteem in Children and Adolescents. In R. F. Baumeister (Ed.), Self-Esteem: The Puzzle of Low SelfRegard (pp. 87-116). New York: Plenum Press.

Haskell, W. L., Yee, M. C., Evans, A., \& Irby, P. J. (1993). Simultaneous measurement of heart rate and body motion to quantitate physical activity. Medicine and Science in Sport and Exercise, 25(1), 109-115.

Heath, G. W., Pratt, M., Warren, C. W., \& Kann, L. (1994). Physical-Activity Patterns in American High-School-Students - Results from the 1990 Youth Risk Behavior Survey. Archives of Pediatrics \& Adolescent Medicine, 148(11), 1131-1136.

Hermes, S. F., \& Keel, P. K. (2003). The influence of puberty and ethnicity on awareness and internalization of the thin ideal. International Journal of Eating Disorders, 33(4), 465-467 URLJ: http://www interscience wiley com/jpages/02763478/.

Heyward, V. H., \& Stolarczyk, L. M. (1996). Applied Body Composition Assessment: Human Kinetics.

Hill, A., \& Roberts, J. (1998). Body mass index: a comparison between selfreported and measured height and weight. Journal of Public Health Medicine, 20(2), 206-210.

Hofferth, S. L., \& Sandberg, J. F. (2001). How American children spend their time. Journal of Marriage and the Family, 63(2), 295-308.

Houtkooper, L. B., Going, S. B., Lohman, T. G., Roche, A. F., \& Van Loan, M. (1992). Bioelectrical impedance estimation of fat-free body mass in children and youth: a cross-validation study. Journal of Applied Physiology, 72(1), 366-373.

Houtkooper, L. B., Lohman, T. G., Going, S. B., \& Hall, M. C. (1989). Validity of bioelectric impedance for body composition assessment in children. Journal of Applied Physiology, 66(2), 814-821.

Howell, D. C. (1997). Statistical Methods for Psychology (4th ed.). Belmont, CA: Duxbury Press.

Howitt, D., \& Cramer, D. (2000). An introduction to statistics in psychology: a complete guide for students (2nd ed.). Harlow: Prentice Hall.

Huddy, D. C., Nieman, D. C., \& Johnson, R. L. (1993). Relationship between body image and percent body fat among college male varsity athletes and nonathletes. Perceptual and Motor Skills, 77(3 Pt 1), 851-857. 
Jackson, A. S., Stanforth, P. R., Gagnon, J., Rankinen, T., Leon, A. S., Rao, D. C., et al. (2002). The effect of sex, age and race on estimating percentage body fat from body mass index: The Heritage Family Study. International Journal of Obesity and Related Metabolic Disorders, 26(6), 789-796.

Janz, K. F. (1994). Validation of the CSA accelerometer for assessing children's physical activity. Medicine and Science in Sports and Exercise, 26(3), 369375 .

Janz, K. F., Dawson, J. D., \& Mahoney, L. T. (2000). Tracking physical fitness and physical activity from childhood to adolescence: the Muscatine study. Medicine and Science in Sports and Exercise, 32(7), 1250-1257.

Janz, K. F., Witt, J., \& Mahoney, L. T. (1995). The stability of children's physical activity as measured by accelerometry and self-report. Medicine and Science in Sports and Exercise, 27(9), 1326-1332.

Jarti, L., Hakanen, M., Paakkunainen, U., Raittinen, P., \& Ronnemaa, T. (2000). Comparison of hand-to-leg and leg-to-leg bioelectric impedance devices in the assessment of body adiposity in prepubertal children. the STRIP study. Special Turku coronary Risk factor Intervention Project. Acta Paediatr, 89(7), 781-786.

Kaplan, K. M., \& Wadden, T. A. (1986). Childhood Obesity and Self-Esteem. Journal of Pediatrics, 109(2), 367-370.

Kimm, S. Y., Barton, B. A., Berhane, K., Ross, J. W., Payne, G. H., \& Schreiber, G. B. (1997). Self-esteem and adiposity in black and white girls: the NHLBI Growth and Health Study. Annals of Epidemiology, 7(8), 550-560.

Kolody, B., \& Sallis, J. F. (1995). A prospective study of ponderosity, body image, self-concept, and psychological variables in children. Journal of Developmental and Behavioral Pediatrics, 16(1), 1-5 URLJ: http://www.jrnldbp.com/.

Korth-Schutz, S., Levine, L. S., \& New, M. I. (1976). Serum androgens in normal prepubertal and pubertal children and in children with precocious adrenarche. The Journal of Clinical Endocrinology and Metabolism, 42(1), 117-124.

Kristjansdottir, G., \& Vilhjalmsson, R. (2001). Sociodemographic differences in patterns of sedentary and physically active behavior in older children and adolescents. Acta Paediatrica, 90(4), 429-435. 
Kuczmarski, R. J., Ogden, C. L., Grummer-Strawn, L. M., Flegal, K. M., Guo, S. S., Wei, R., et al. (2000). CDC growth charts: United States. Advance Data(314), 127.

Laberge, L., Petit, D., Simard, C., Vitaro, F., Tremblay, R. E., \& Montplaisir, J. (2001). Development of sleep patterns in early adolescence. Journal of Sleep Research, 10(1), 59-67.

Lahey, M. A., Downey, R. G., \& Saal, F. E. (1983). Intraclass correlations: There's more there than meets the eye. Psychological Bulletin, 93(3), 586-595.

Lawrence, F. C., Tasker, G. E., Daly, C. T., Orhiel, A. L., \& Wozniak, P. H. (1986). Adolescents' time spent viewing television. Adolescence, 21(82), 431-436.

Lintunen, T., Rahkila, P., Silvennoinen, M., \& Österback, L. (1988). Psychological and Physical Correlates of Early and Late Biological Maturation in 9to 11-Year-Old Girls and Boys. In R. M. Malina (Ed.), Young Athletes: Biological, Psychological and Educational Perspectives (pp. 85-91). Champaign, Illinois: Human Kinetics.

Lobstein, T. J., James, W. P., \& Cole, T. J. (2003). Increasing levels of excess weight among children in England. International Journal of Obesity and Related Metabolic Disorders, 27(9), 1136-1138.

Lohman, T. G. (1981). Skinfolds and Body Density and Their Relation to Body Fatness: A Review. Human Biology,-53(2), 181-225.

Lohman, T. G. (1992). Advances in Body Composition Assessment, Current Issues in Exercise Science Series. Champaign, Illinois: Human Kinetics.

Lohman, T. G., Roch, A. F., \& Martorell, R. (Eds.). (1988). Anthropometric Standardization Reference Manual. Champaign, Illinois: Human Kinetics Books.

Lukaski, H. C., Johnson, P. E., Bolonchuk, W. W., \& Lykken, G. I. (1985). Assessment of fat-free mass using bioelectrical impedance measurements of the human body. American Journal of Clinical Nutrition, 41, 810-817.

Maffeis, C., Talamini, G., \& Tato, L. (1998). Influence of diet, physical activity and parents' obesity on children's adiposity: a four-year longitudinal study. International Journal of Obesity and Related Metabolic Disorders, 22(8), 758-764.

Marsh, H. W., \& Redmayne, R. S. (1994). A multidimensional physical selfconcept and its relations to multiple components of physical fitness. Journal of Sport and Exercise Psychology, 16(1), 43-55. 
Marsh, H. W., Richards, G. E., Johnson, S., Roche, L., \& Tremayne, P. (1994). Physical Self-Description Questionnaire: Psychometric properties and a multitraitmultimethod analysis of relations to existing instruments. Journal of Sport and Exercise Psychology, 16(3), 270-305.

Marshall, S. J. (2001). From Runner Bean to Coach Potato. Unpublished Doctoral Dissertation, Loughborough University.

Marshall, S. J., Biddle, S. J. H., Sallis, J. F., McKenzie, T. L., \& Conway, T. L. (2002). Clustering of sedentary Behaviors and physical activity among youth: A cross-national study. Pediatric Exercise Science, 14(4), 401-417.

Marshall, W. A., \& Tanner, J. M. (1969). Variations in pattern of pubertal changes in girls. Archives of Disease in Children, 44(235), 291-303.

Marshall, W. A., \& Tanner, J. M. (1970). Variations in the pattern of pubertal changes in boys. Archives of Disease in Children, 45(239), 13-23.

Martinez-Gonzalez, M. A., Martinez, J. A., Hu, F. B., Gibney, M. J., \& Kearney, J. (1999). Physical inactivity, sedentary lifestyle and obesity in the European Union. International Journal of Obesity and Related Metabolic Disorders, 23(11), 1192-1201.

Matsudo, S. M. M., \& Matsudo, V. K. R. (1994). Self-Assessment and Physician Assessment of Sexual-Maturation in Brazilian Boys and Girls Concordance and Reproducibility. American Journal of Human Biology, 6(4), 451455.

McCabe, M. P., \& Ricciardelli, L. A. (2003). Sociocultural influences on body image and body changes among adolescent boys and girls. Journal of Social Psychology, 143(1), 5-26.

McCabe, M. P., Ricciardelli, L. A., \& Finemore, J. (2002). The role of puberty, media and popularity with peers on strategies to increase weight, decrease weight and increase muscle tone among adolescent boys and girls. Journal of Psychosomatic Research, 52(3), 145-153.

McCarthy, H. D., Ellis, S. M., \& Cole, T. J. (2003). Central overweight and obesity in British youth aged 11-16 years: cross sectional surveys of waist circumference. British Medical Journal, 326(7390), 624.

McGraw, K. O., \& Wong, S. P. (1996). Forming inferences about some intraclass correlation coefficients. Psychological Methods, 1(1), 30-46. 
McKenzie, T. L., Marshall, S. J., Sallis, J. F., \& Conway, T. L. (2000). Leisure-time physical activity in school environments: An observational study using SOPLAY. Preventive Medicine, 30(1), 70-77.

Mirwald, R. L., \& Bailey, D. A. (1986). Maximal Aerobic Power. London, Ontario: Sports Dynamics.

Montoye, H. J., Washburn, R., Servais, S., Ertl, A., Webster, J. G., \& Nagle, F. J. (1983). Estimation of energy expenditure by a portable accelerometer. Medicine and Science in Sports and Exercise, 15(5), 403-407.

Mueller, W. H., Dai, S., \& Labarthe, D. R. (2001). Tracking body fat distribution during growth: using measurements at two occasions vs one. International Journal of Obesity and Related Metabolic Disorders, 25(12), 1850-1855.

Myers, L., Strikmiller, P. K., Webber, L. S., \& Berenson, G. S. (1996). Physical and sedentary activity in school children grades 5-8: the Bogalusa Heart Study. Med Sci Sports Exerc, 28(7), 852-859.

National Heart Lung and Blood Institute. (1998a). Clinical Guidelines on the Identification, Evaluation. and Treatment of Overweight and Obesity in Adults. The Evidence Report [On-Line]. Retrieved, from the World Wide Web: http://www.nhlbi.nih.gov/guidelines/obesity/ob gdlns.pdf

National Heart Lung and Blood Institute. (1998b). First Federal Obesity Clinical Guidelines Released [On-Line]. National Institutes of Health News Release. Retrieved, from the World Wide Web: http://www.nhlbi.nih.gov/news/press/oberel4f.htm

Neinstein, L. S. (1982). Adolescent Self-Assessment of Sexual-Maturation Reassessment and Evaluation in a Mixed Ethnic Urban-Population. Clinical Pediatrics, 21(8), 482-484.

Neyzi, O., Alp, H., Yalcindag, A., Yakacikli, S., \& Orphon, A. (1975). Sexual Maturation in Turkish Boys. Annals of Human Biology, 2(3), 251-259.

Nichols, D. P. (1998). Choosing an intraclass correlation coefficient. SPSS Keywords, Number 67. Retrieved, from the World Wide Web: www.spss.com/tech/stat/articles/whichicc.txt

Norgan, N. G., \& Jones, P. R. M. (1995). The Effect of Standardizing the Body-Mass Index for Relative Sitting Height. International Journal of Obesity, 19(3), 206-208. 
Nunez, C., Gallagher, D., Visser, M., Pisunyer, F. X., Wang, Z. M., \& Heymsfield, S. B. (1997). Bioimpedance analysis: Evaluation of leg to leg system based on pressure contact foot pad electrodes. Medicine and Science in Sports and Exercise, 29(4), 524-531.

Owen, N., Leslie, E., Salmon, J., \& Fotheringham, M. J. (2000). Environmental determinants of physical activity and sedentary behavior. Exercise and Sport Sciences Reviews, 28(4), 153-158.

Page, A., \& Fox, K. R. (1997). Adolescent Weight Management and The Physical Self. In K. R. Fox (Ed.), The Physical Self: from motivation to well-being (pp. 229-256). Champaign, Illinois: Human Kinetics.

Pate, R. R., Pratt, M., Blair, S. N., Haskell, W. L., Macera, C. A., Bouchard, C., et al. (1995). Physical activity and public health. A recommendation from the Centers for Disease Control and Prevention and the American College of Sports Medicine. JAMA, 273(5), 402-407.

Phillips, C., Rolls, S., Rouse, A., \& Griffiths, M. (1995). Home video game playing in schoolchildren: a study of incidence and patterns of play. Journal of Adolescence, 18(6), 687.

Pietrobelli, A., Faith, M. S., Allison, D. B., Gallagher, D., Chiumello, G., \& Heymsfield, S. B. (1998). Body mass index as a measure of adiposity among children and adolescents: a validation study. Journal of Pediatrics, 132(2), 204-210.

Pratt, M., Macera, C. A., \& Blanton, C. (1999). Levels of physical activity and inactivity in children and adults in the United States: current evidence and research issues. Medicine and Science in Sports and Exercise, 31(11 Suppl), S526-533.

Raitakari, O. T., Porkka, K. V., Taimela, S., Telama, R., Rasanen, L., \& Viikari, J. S. (1994). Effects of persistent physical activity and inactivity on coronary risk factors in children and young adults. The Cardiovascular Risk in Young Finns Study. American Journal of Epidemiology, 140(3), 195-205.

Reilly, J. J., \& Dorosty, A. R. (1999). Epidemic of obesity in UK children. Lancet, 354(9193), 1874-1875.

Ricciardelli, L. A., \& McCabe, M. P. (2003). A longitudinal analysis of the role of biopsychosocial factors in predicting body change strategies among adolescent boys. Sex Roles, 48(7-8), 349-359.

Robinson, T. N., Hammer, L. D., Killen, J. D., Kraemer, H. C., Wilson, D. M., Hayward, C., et al. (1993). Does Television Viewing Increase Obesity and Reduce 
Physical- Activity - Cross-Sectional and Longitudinal Analyses among Adolescent Girls. Pediatrics, 91(2), 273-280.

Rogol, A. D., Clarke, P. A., \& Roemmich, J. N. (2000). Growth and pubertal development in children and adolescents: effects of diet and physical activity. American Journal of Clinical Nutrition, 72(2), 521s-528s.

Rogol, A. D., Roemmich, J. N., \& Clark, P. A. (2002). Growth at puberty. Journal of Adolescent Health, 31(6 Suppl), 192-200.

Sallis, J. F. (1991). Self-Report Measures of Children's Physical Activity. Journal of School Health, 6I(5), 215-219.

Sallis, J. F., Buono, M. J., Roby, J. J., Micale, F. G., \& Nelson, J. A. (1993). Seven-day recall and other physical activity self-reports in children and adolescents. Medicine and Science in Sports and Exercise, 25(1), 99-108.

Sallis, J. F., \& Owen, N. (1996). Ecological Models. In K. Glanz \& F. M. Lewis \& B. K. Rimer (Eds.), Health Behavior and Health Education. San Francisco: Jossey-Bass Publishers.

Sallis, J. F., \& Patrick, K. (1994). Physical activity guidelines for adolescents:consensus statement. Pediatric Exercise Science, 6, 302-314.

Sallis, J. F., Simons Morton, B. G., Stone, E. J., Corbin, C. B., \& et al. (1992). Determinants of physical activity and interventions in youth. Medicine and Science in Sports and Exercise, Vol 24(6, Suppl), S248-S257.

Sallis, J. F., Simons-Morton, B. G., Stone, E. J., Corbin, C. B., Epstein, L. H., Faucette, N., et al. (1992). Determinants of physical activity and interventions in youth. Medicine and Science in Sports and Exercise, 24(6 Suppl), S248-257.

Salmon, J., Bauman, A., Crawford, D., Timperio, A., \& Owen, N. (2000). The association between television viewing and overweight among Australian adults participating in varying levels of leisure-time physical activity. International Journal of Obesity and Related Metabolic Disorders, 24(5), 600-606.

Sands, E. R., \& Wardle, J. (2003). Internalization of ideal body shapes in 9-12year-old girls. International Journal of Eating Disorders, 33(2), 193-204 URLJ: http://www.interscience.wiley.com/jpages/0276-3478/.

Sarkin, J. A., Nichols, J. F., Sallis, J. F., \& Calfas, K. J. (2000). Self-report measures and scoring protocols affect prevalence estimates of meeting physical activity guidelines. Medicine and Science in Sports and Exercise, 32(1), 149-156. 
Schaefer, F., Georgi, M., Zieger, A., \& Scharer, K. (1994). Usefulness of bioelectric impedance and skinfold measurements in predicting fat-free mass derived from total body potassium in children. Pediatr Res, 35(5), 617-624.

Schmitz, K. H., Lytle, L. A., Phillips, G. A., Murray, D. M., Birnbaum, A. S., \& Kubik, M. Y. (2002). Psychosocial correlates of physical activity and sedentary leisure habits in young adolescents: the Teens Eating for Energy and Nutrition at School study. Preventive Medicine, 34(2), 266-278.

Segal, K. R., Gutin, B., Presta, E., Wang, J., \& Van Itallie, T. B. (1985). Estimation of human body composition by electrical impedance methods: a comparative study. Journal of Applied Physiology, 58(5), 1565-1571.

Segal, K. R., Van Loan, M., Fitzgerald, P. I., Hodgdon, J. A., \& Van Itallie, T. B. (1988). Lean body mass estimation by bioelectrical impedance analysis: a four-site cross validation study. American Journal of Clinical Nutrition, 47, 7-14.

Shann, M. H. (2001). Students' use of time outside of school: A case for after school programs for urban middle school youth. Urban Review, 33(4), 339-356.

Shrout, P. E., \& Fleiss, J. L. (1979). Intraclass correlations: Uses in assessing rater reliability. Psychological Bulletin, 86(2), 420-428.

Sirard, J. R., \& Pate, R. R. (2001). Physical activity assessment in children and adolescents. Sports Medicine, 31(6), 439-454.

Slaughter, M. H., Lohman, T. G., Boileau, R. A., Horswill, C. A., Stillman, M. D., Van Loan, M. D., et al. (1988). Skinfold Equations for Estimation of Body Fatness in Children and Youth. Human Biology, 60(5), 709-723.

Sonstroem, R. J. (1997). The Physical Self-System: A Mediator of Exercise and Self-Esteem. In K. R. Fox (Ed.), The Physical Self: from motivation to well-being (pp. 3-26). Champaign, Illinois: Human Kinetics.

Stokols, D. (1992). Establishing and maintaining healthy environments. Toward a social ecology of health promotion. The American Psychologist, 47(1), 6 22.

Tanner, J. M. (1962). Growth at Adolescence (2nd ed.). Oxford: Blackwell Scientific Publications.

Tanner, J. M. (1989). Foetus into Man: Physical Growth from Conception to Maturity (2 ed.). Ware: Castlemead Publications. 
Tanner, J. M., Whitehouse, R. H., Marubini, E., \& Resele, L. F. (1976). The adolescent growth spurt of boys and girls of the Harpenden growth study. Annals of Human Biology, 3(2), 109-126.

Taylor, R. W., Jones, I. E., Williams, S. M., \& Goulding, A. (2002). Body fat percentages measured by dual-energy X-ray absorptiometry corresponding to recently recommended body mass index cutoffs for overweight and obesity in children and adolescents aged 3-18 y. American Journal of Clinical Nutrition, 76(6), 1416-1421.

Taylor, W. C., Yancey, A. K., Leslie, J., Murray, N. G., Cummings, S. S., Sharkey, S. A., et al. (1999). Physical activity among African American and Latino middle school girls: consistent beliefs, expectations, and experiences across two sites. Women and Health, 30(2), 67-82.

Tremblay, M. S., \& Willms, J. D. (2003). Is the Canadian childhood obesity epidemic related to physical inactivity? International Journal of Obesity and Related Metabolic Disorders, 27(9), 1100-1105.

Trost, S. G., Ward, D. S., Moorehead, S. M., Watson, P. D., Riner, W., \& Burke, J. R. (1998). Validity of the computer science and applications (CSA) activity monitor in children. Medicine and Science in Sports and Exercise, 30(4), 629-633.

Tudor-Locke, C., Neff, L. J., Ainsworth, B. E., Addy, C. L., \& Popkin, B. M. (2002). Omission of active commuting to school and the prevalence of children's health-related physical activity levels: The Russian Longitudinal Monitoring Study. Child: Care, Health and Development, 28(6), 507-512 URLJ: http://www blackwellscience $\mathrm{com} /$-cgilib/jnlpage asp?Journal=cchd\&File=cchd.

Tudor-Locke, C. E., \& Myers, A. M. (2001). Challenges and opportunities for measuring physical activity in sedentary adults. Sports Medicine, 31(2), 91-100.

Tyrrell, V. J., Richards, G., Hofman, P., Gillies, G. F., Robinson, E., \& Cutfield, W. S. (2001a). Foot-to-foot bioelectrical impedance analysis: a valuable tool for the measurement of body composition in children. International Journal of Obesity, 25(2), 273-278.

Tyrrell, V. J., Richards, G. E., Hofman, P., Gillies, G. F., Robinson, E., \& Cutfield, W. S. (2001b). Obesity in Auckland school children: a comparison of the body mass index and percentage body fat as the diagnostic criterion. International Journal of Obesity and Related Metabolic Disorders, 25(2), 164-169. 
U.S. Department of Health and Human Services. (1996). Physical Activity and Health: A Report of the Surgeon General. Atlanta, GA: U.S. Department of Health and Human Services.

Van den Bulck, J. (2000). Is television bad for your health? Behavior and body image of the adolescent "couch potato". Journal of Youth and Adolescence, 29(3), 273-288.

Van Loan, M., \& Mayclin, P. (1987). Bioelectrical Impedance Analysis: Is It a Reliable Estimator of Lean Body Mass and Total Body Water. Human Biology, 59(2), 299-309.

Vuchinich, R. E., \& Tucker, J. A. (1988). Contributions from behavioral theories of choice to an analysis of alcohol abuse. J Abnorm Psychol, 97(2), 181-195.

Wadden, T. A., Brown, G., Foster, G. D., \& Linowitz, J. R. (1991). Salience of weight-related worries in adolescent males and females. International Journal of Eating Disorders, 10(4), 407-414.

Wagner, D. R., \& Heyward, V. H. (2001). Validity of two-component models for estimating body fat of black men. Journal of Applied Physiology, 90(2), 649-656.

Waller, C. W., \& Popkin, B. M. (2002). Television viewing, video games, and studying are not associated with overweight in Chinese children. Faseb Journal, 16(5), A1020-A1020.

Wang, J., Thornton, J. C., Russell, M., Burastero, S., Heymsfield, S., \& Pierson Jr, R. N. (1994). Asians have lower body mass index (BMI) but higher percent body fat than do whites: comparisons of anthropometric measurements. American Journal of Clinical Nutrition, 60, 23-28.

Waters, E. B., \& Baur, L. A. (2003). Childhood obesity: modernity's scourge. Medical Journal of Australia, 178(9), 422-423.

Whitehead, J. R. (1995). A Study of Children's Physical Self-Perceptions Using an Adapted Physical Self-Perception Profile Questionnaire. Pediatric Exercise Science, 7, 132-151.

Widhalm, K., Schönegger, K., Huemer, C., \& Auterith, A. (2001). Does the BMI reflect body fat in obese children and adolescents? A study using the TOBEC method. International Journal of Obesity, 25, 279-285.

Williams, C. D., Sallis, J. F., Calfas, K. J., \& Burke, R. (1999). Psychosocial and demographic correlates of television viewing. American Journal of Health Promotion, 13(4), 207-214. 
Wolf, A. M., Gortmaker, S. L., Cheung, L., Gray, H. M., Herzog, D. B., \& Colditz, G. A. (1993). Activity, inactivity, and obesity: racial, ethnic, and age differences among schoolgirls. Am J Public Health, 83(11), 1625-1627.

Wolfson, A. R., \& Carskadon, M. A. (1998). Sleep schedules and daytime functioning in adolescents. Child Development, 69(4), 875-887.

Wong, T. C., Webster, J. G., Montoye, H. J., \& Washburn, R. (1981). Portable accelerometer device for measuring human energy expenditure. IEEE Transactions on Bio-Medical Engineering, 28(6), 467-471.

Zhang, K., Werner, P., Sun, M., Pi-Sunyer, F. X., \& Boozer, C. N. (2003). Measurement of human daily physical activity. Obesity Research, 11(1), 33-40. 


\section{Appendix 1: Data Cleaning}

\section{a) Anthropometric Data}

A variety of methods were used to search for errors. Any measurements considered unreliable due to excess adiposity were removed prior to any analysis. Displaying frequency tables of each variable in order to indicate any invalid or extreme entries identified univariate outliers. Multivariate outliers were identified by creating flag variables that indicated when the two measures for each variable differed by more than the previously calculated measurement limits. If any extreme differences were identified then the raw data was checked for any entry errors.

Finally, ten percent of the measurement files were randomly extracted, checked against the raw data and corrected where necessary. An acceptable limit of one percent entry error was set, if this was exceeded then the data was cleaned, returned and another sample of ten percent extracted. This process was repeated until a set of files was extracted that contained less then one percent entry errors.

\section{b) Demographic Data}

Univariate outliers were identified as above. Creating flag variables that indicated when unusual or unacceptable combinations of variables were present identified multivariate outliers. Entry errors were corrected as for anthropometric data.

\section{c) Physical Self-Perception Profile for Children (C-PSPP)}

All files were double checked against the original data for entry errors. Students who returned questionnaires that indicated that they had not understood the task, e.g., they had chosen multiple options for each question, had their data removed before analysis. 
If participants had made obvious errors of reporting then these were corrected during the data entry. For example, if a student had reported walking home from school for two time periods ( 30 minutes) but also reported that they were in category 11(Other inside area) then this was assumed to be an error and the 'Where?' variable was changed from category 11 to category 12 (Other outside area). Univariate outliers were assessed as previously described and finally a selection of ten percent of the entries were extracted and checked as for the anthropometric data above. 


\section{Appendix 2: Ecological \\ Questionnaire and Example Day From The Free-Time Diary}


1. About You:

1. Your name:

2. 10.11 .56 .1 .0 .1

3. Sex:

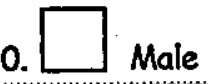

1. $\square$ Female

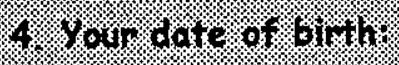

5. Your school year (circle one):

$7^{\text {th }} \quad 8^{\text {th }} \quad 9^{\text {th }} \quad 10^{\text {th }} \quad 11^{\text {th }}$

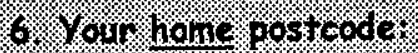

7a. How would you describe 1

1. $\square$ Black-Caribbean

2.

Black-African yourself? (tick one):

3. White-European

4. $\square$ Asian

5. $\square$ Chinese

6. $\square$ Other (please describe):

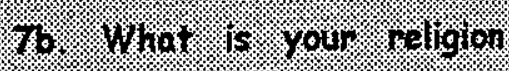

$\sqrt[1]{6}+1010$

(ticl. ones?

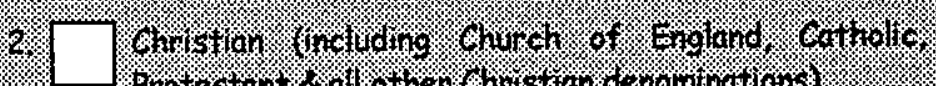

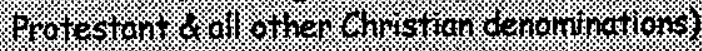

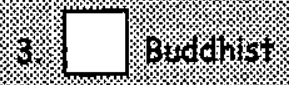

.

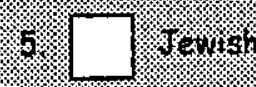

6.

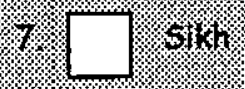

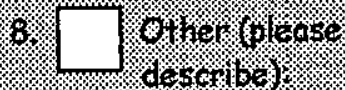

\section{Have you started puberty?}

(e.g., do you have any pubic hair?)

0.

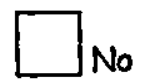

1.

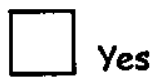

2. Not sure

\section{GRRLS:}

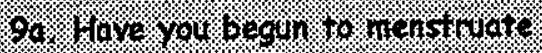

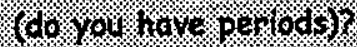

0.

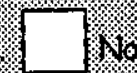

1.

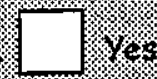

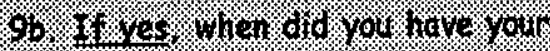

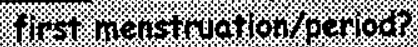

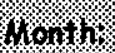

Teor.

\section{BOYS:}

10a. Has your voice broken? (i.e., do you speak. with a deeper voice than when you

o.<smiles>[Mg][C]1CCCC1</smiles>

1. $\square$ Yes were younger?

10b. If yes, when did you first notice that your voice was deeper?

Month: Year:

10c. Have you started to shave?

If yes, how many times per month?

0.

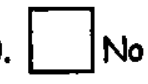

1.

Yes, times/month 


\section{About Your Family:}

11. How many people live in your house (including you)?

1.

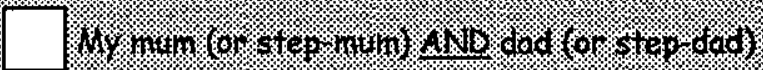

120. W10.00. .00, 1V. WItis?

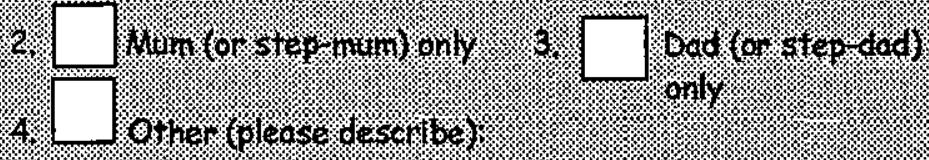

Mother (or step-mother):

12b. What are their occupations?:

Father (or step-father):

Other:

15. How nany brothers and 31518 .

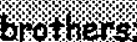
itstents:

Brothers:

1.

2.

3.

4.
Sisters:

1.

2.

3.

4.

The next three questions refer to your birthl You will need to ASK your MUM OR DAD for the answerl

\begin{tabular}{|ll|}
\hline $\begin{array}{ll}\text { 15a. How long had your mum been } \\
\text { pregnant with you when you were born? }\end{array}$ & 2. $\square$ Less than 37 weeks (Pre-term) \\
& 37-41 wks (Full-term) \\
More than 41 weeks (Post-term) \\
$\begin{array}{ll}\text { 15b. If you were pre-term or post-term, } \\
\text { how many weeks of pregnancy had your }\end{array}$ \\
mother completed when you were born?
\end{tabular}




\section{About Your Home:}

16. How many rooms does your home have?

$x$ Do NOT count bathrooms, toilets, halls or landings, or rooms that can only be used for storage such as cupboards.

No. of rooms

$\checkmark$ Do count all other rooms, for example kitchens, living rooms, bedrooms, utllity rooms and studies. If two rooms have been converted into one room count them as ONE room

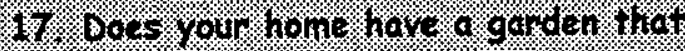
col can ploy in?

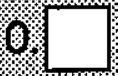

10

1.

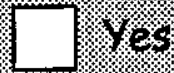

18. Is your home on a busy road?

0

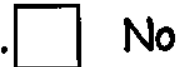

1. $\square$ Yes

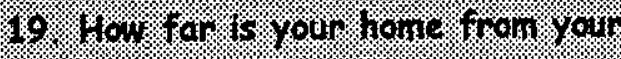
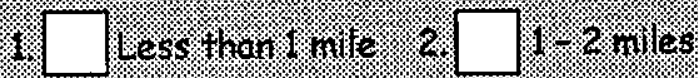
$80.1001 ?$

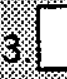
2. $3.1711 \mathrm{es}$

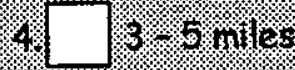

5.

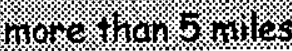

20. Please indicate how many of the following items can be found in your home? (please circle):

\section{a. Televisions}

20a. In your home
(including your bedroom)

20b. How many of these are in your bedroom?

b. Video machines

$\begin{array}{lllllll}0 & 1 & 2 & 3 & 4 & 5 & 6+\end{array}$

$\begin{array}{lllllll}0 & 1 & 2 & 3 & 4 & 5 & 6+\end{array}$

$\begin{array}{lllllll}0 & 1 & 2 & 3 & 4 & 5 & 6+\end{array}$

$\begin{array}{lllllll}0 & 1 & 2 & 3 & 4 & 5 & 6+\end{array}$

c. Video Game Players (e.g.. PS2, N64 etc)

$\begin{array}{lllllll}0 & 1 & 2 & 3 & 4 & 5 & 6+\end{array}$

d. Telephones (NOT mobiles)

$\begin{array}{lllllll}0 & 1 & 2 & 3 & 4 & 5 & 6+\end{array}$

$\begin{array}{lllllll}0 & 1 & 2 & 3 & 4 & 5 & 6+\end{array}$

$\begin{array}{lllllll}0 & 1 & 2 & 3 & 4 & 5 & 6+\end{array}$

e. Computers with internet access

$\begin{array}{lllllll}0 & 1 & 2 & 3 & 4 & 5 & 6+\end{array}$

$\begin{array}{lllllll}0 & 1 & 2 & 3 & 4 & 5 & 6+\end{array}$

f. Computers without internet access

$\begin{array}{lllllll}0 & 1 & 2 & 3 & 4 & 5 & 6+\end{array}$

$\begin{array}{lllllll}0 & 1 & 2 & 3 & 4 & 5 & 6+\end{array}$

g. Music Stereos (including portables)

$\begin{array}{lllllll}0 & 1 & 2 & 3 & 4 & 5 & 6+\end{array}$

$\begin{array}{lllllll}0 & 1 & 2 & 3 & 4 & 5 & 6+\end{array}$

75. Do. gou own any of the rollowing

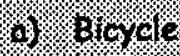<smiles>O=NC1CCC1=O</smiles>

.1.

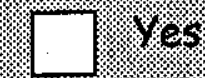

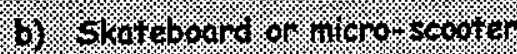

\% 10

1.

1.105

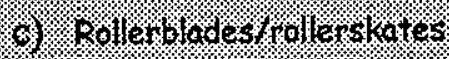

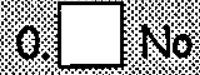

1.

.

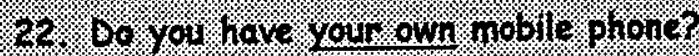

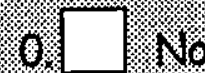

1.

Yes 


\section{SCHOOL DAY 1 General Comments}

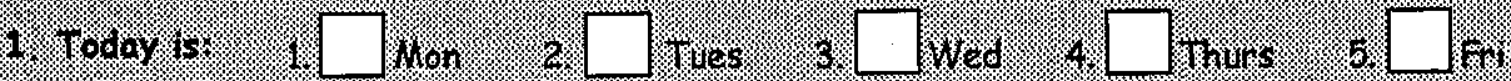

2. The temperature outside today is: $1 . \square$ Very cold $\quad 2 . \square$ cold $\quad 3 . \square$ Mild (tick one)
4.
Warm
5. Hot

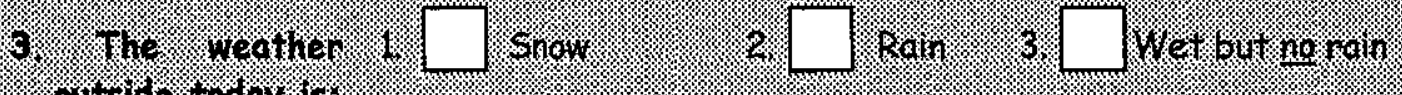

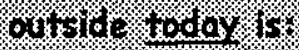
tion ones)

\section{COMPLETE THE FOLLOWING QUESTIONS AT THE END OF THE DAY}

4. Did you take part in a PE lesson today?

$0 . \square$ No

1. $\square$ Yes

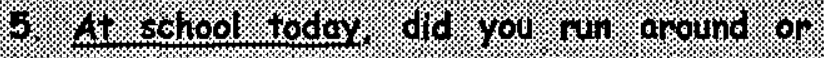

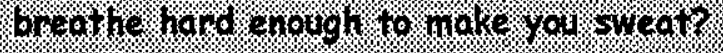

(0.

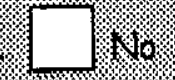

1.

6. Which of the following main meals have

1. $\square$ Breakfast

2. $\square$ Lunch you eaten today? (tick all that apply)

3. Evening Dinner

4. $\square$

None

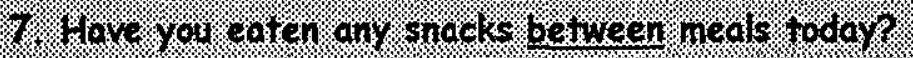

o.

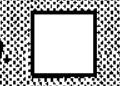

10.

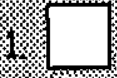

16.

Today, I have eaten the following snacks between meals....... (please circle a number)

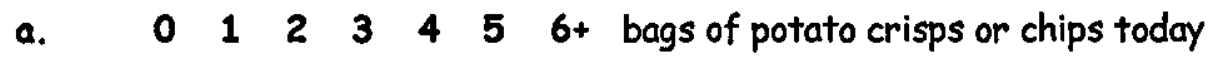
b. $\quad \begin{array}{lllllll}0 & 1 & 2 & 3 & 4 & 5 & 6+\text { bars of chocolate or sweets }\end{array}$
c. $\quad \begin{array}{lllllll}0 & 1 & 2 & 3 & 4 & 5 & 6+\text { pieces of fruit or vegetables }\end{array}$

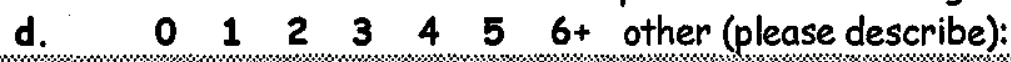

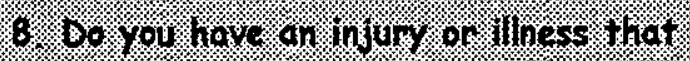

\%

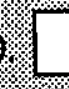

1.

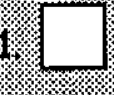

185

it thected whit you have done 1odid?

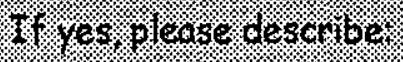




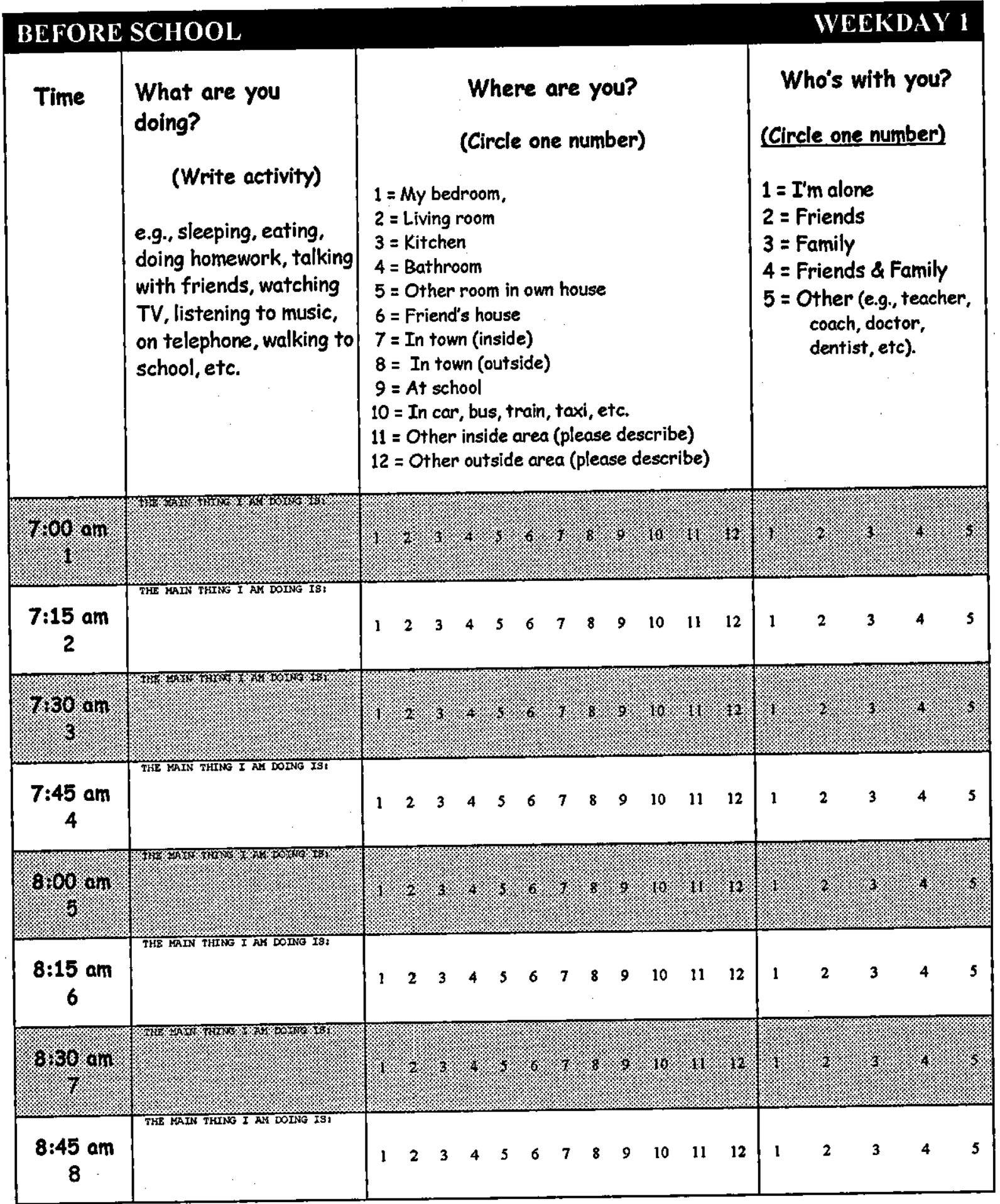

Yoa do not need to record daring school time, please continae aftere school has finished - thank you. 


\section{AFTER SCHOOL}

WEEKDAY 1

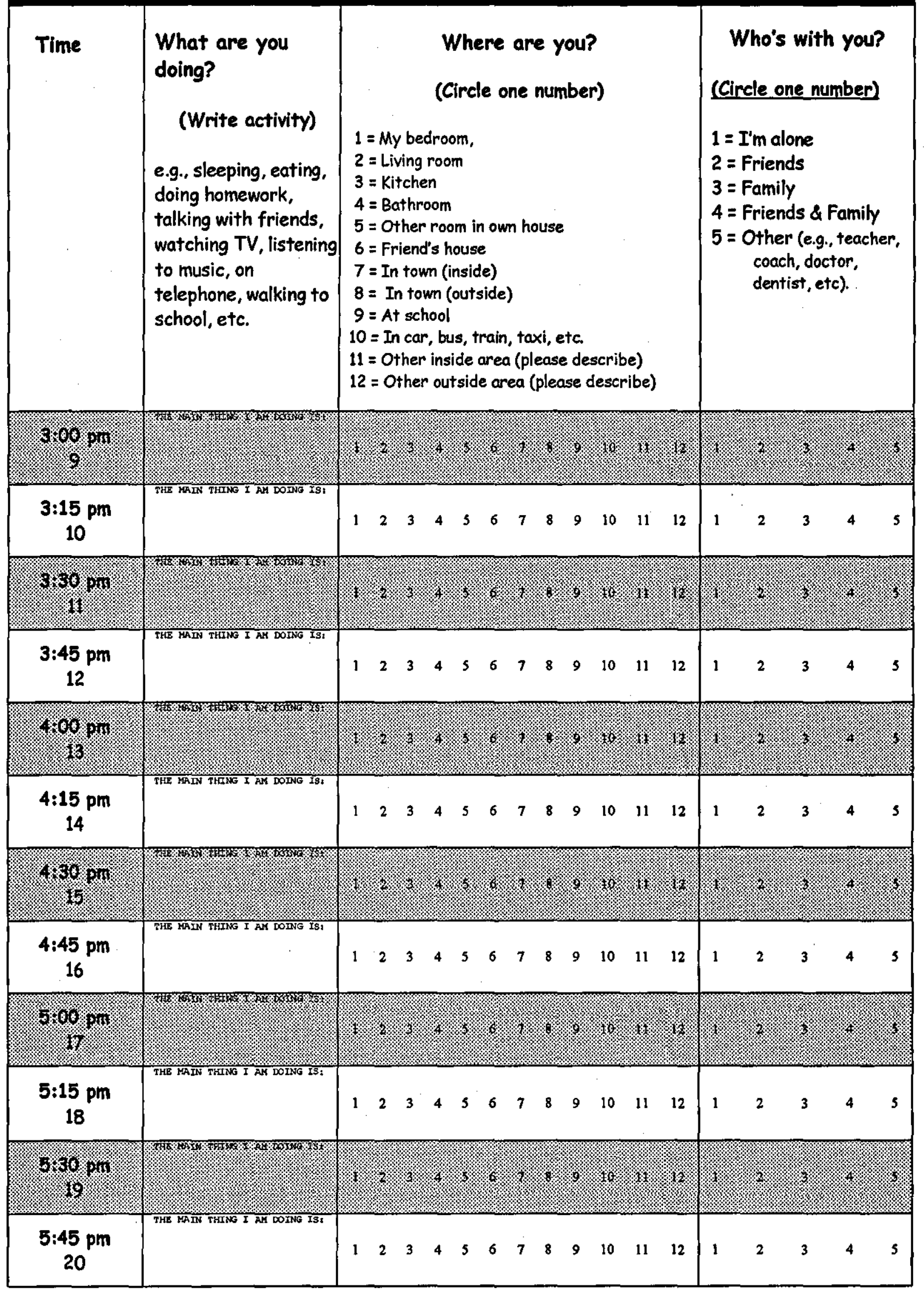




\section{AFTER SCHOOL}

WEEKDAY 1

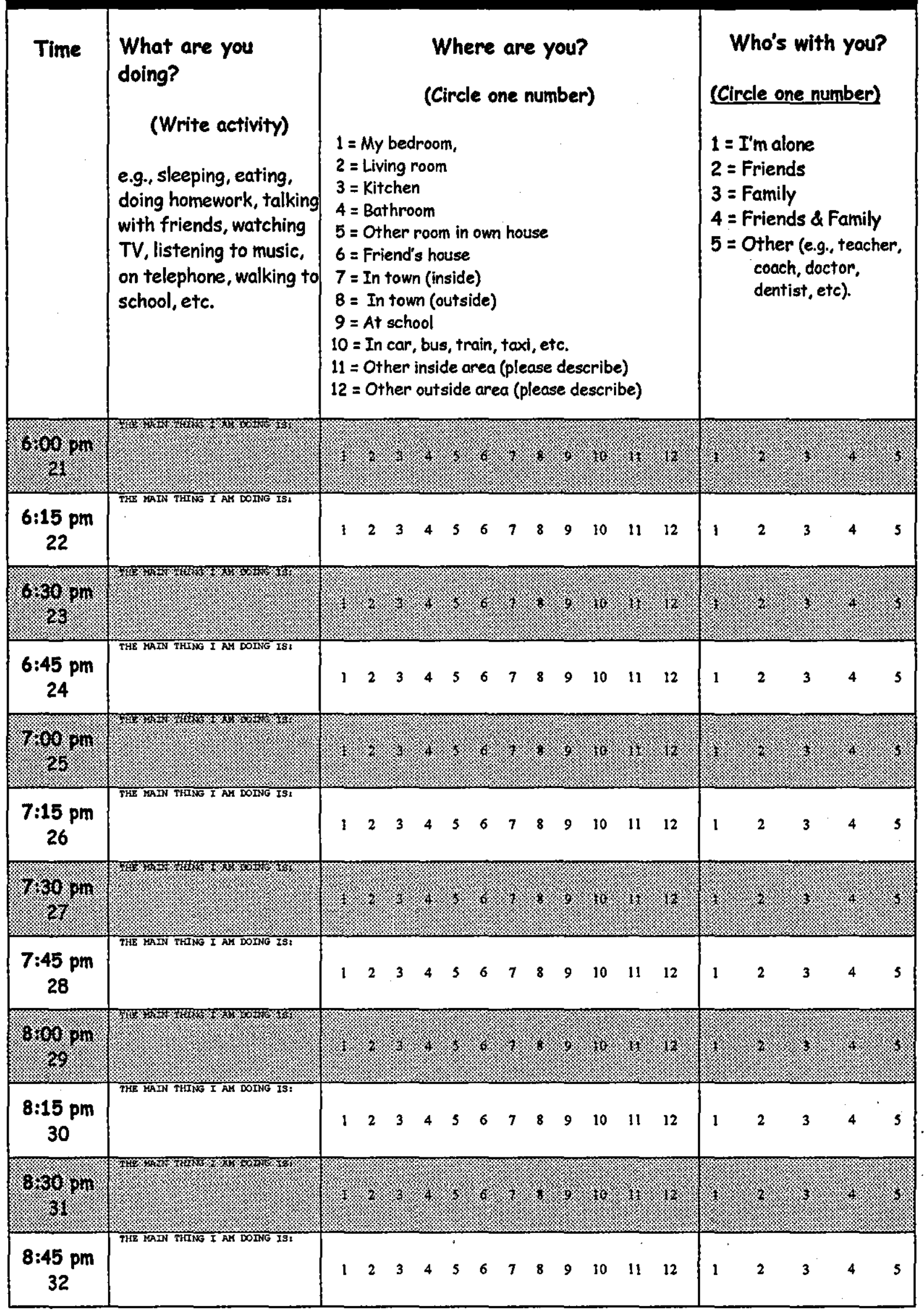




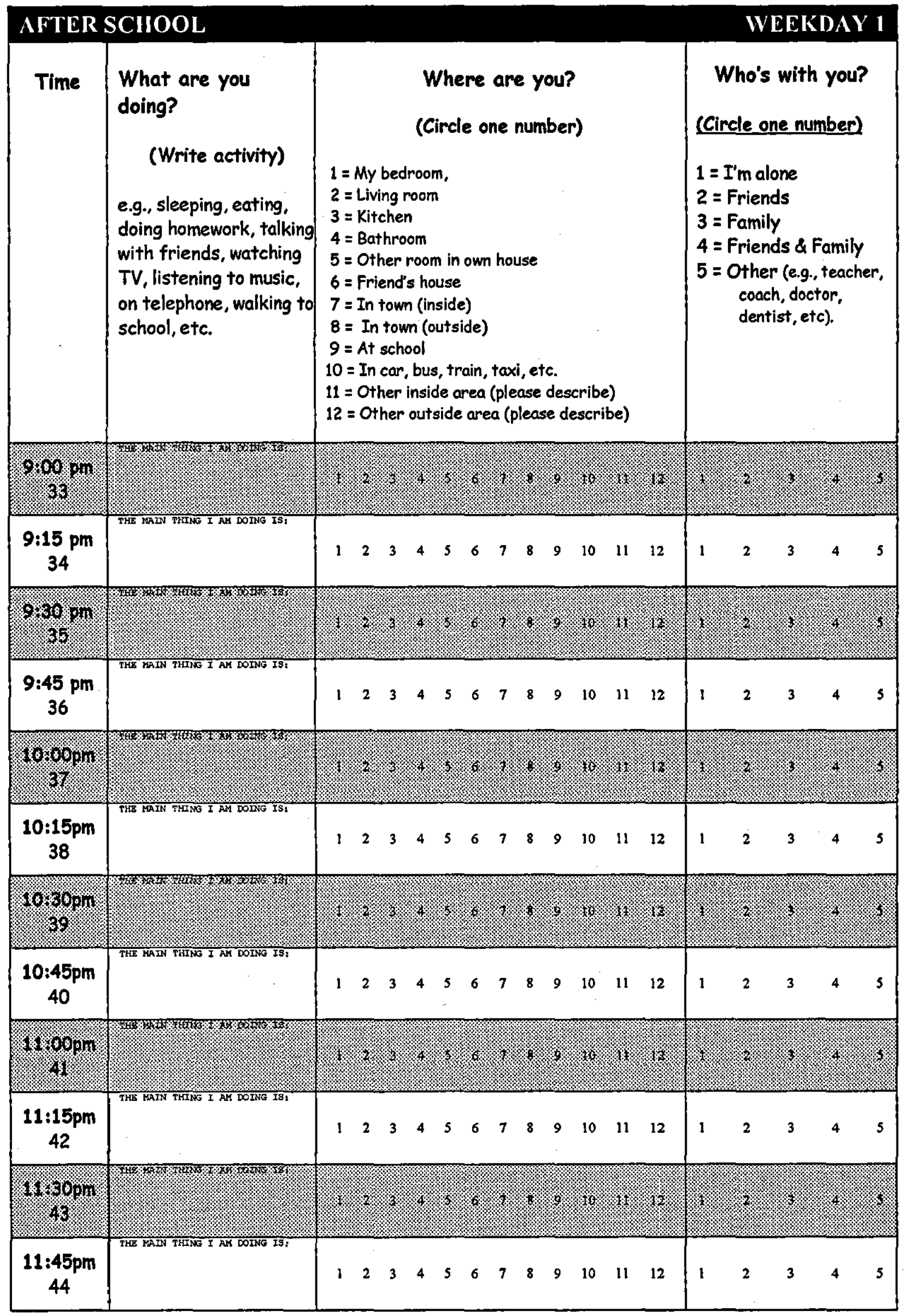




\section{Appendix 3: Physical Self-Perception Profile for Children}




\begin{tabular}{|c|c|c|c|c|c|c|c|}
\hline \multicolumn{8}{|c|}{ What are you like? } \\
\hline & $\begin{array}{l}\text { Really } \\
\text { true for } \\
\text { me }\end{array}$ & $\begin{array}{l}\text { Sort of } \\
\text { true for } \\
\text { me }\end{array}$ & \multicolumn{3}{|c|}{ Remember: only tick ONE box for each question please } & $\begin{array}{l}\text { Sort of } \\
\text { true for }\end{array}$ & $\begin{array}{l}\text { Really } \\
\text { true for }\end{array}$ \\
\hline 1 & & & $\begin{array}{l}\text { Some young people feel that they have a } \\
\text { good-looking (fit-looking) body compared } \\
\text { to others }\end{array}$ & BUT & $\begin{array}{l}\text { Other young people feel that compared to } \\
\text { most, their body doesn't look so good. }\end{array}$ & & \\
\hline 2 & & & $\begin{array}{l}\text { Some young people are proud of } \\
\text { themselves physically }\end{array}$ & BUT & $\begin{array}{l}\text { Other young people don't have much to be } \\
\text { proud of physically. }\end{array}$ & & \\
\hline 3 & & & $\begin{array}{l}\text { Some young people are often unhappy with } \\
\text { themselves }\end{array}$ & BUT & $\begin{array}{l}\text { Other young people are pretty pleased with } \\
\text { themselves. }\end{array}$ & & \\
\hline 4 & & & $\begin{array}{l}\text { Some young people find it difficult to keep } \\
\text { their bodies looking good physically }\end{array}$ & BUT & $\begin{array}{l}\text { Other young people find it easy to keep their } \\
\text { bodies looking good physically. }\end{array}$ & & \\
\hline 5 & & & $\begin{array}{l}\text { Some young people don't feel very } \\
\text { confident about themselves physically }\end{array}$ & BUT & $\begin{array}{l}\text { Other young people really feel good about } \\
\text { themselves physically. }\end{array}$ & & \\
\hline 6 & & & $\begin{array}{l}\text { Some young people are happy with } \\
\text { themselves as a person }\end{array}$ & BUT & $\begin{array}{l}\text { Other young people are often not happy with } \\
\text { themselves. }\end{array}$ & & \\
\hline 7 & & & $\begin{array}{l}\text { Some young people are pleased with the } \\
\text { appearance of their bodies }\end{array}$ & BUT & $\begin{array}{l}\text { Other young people wish that their bodies } \\
\text { looked in better shape physically. }\end{array}$ & & \\
\hline 8 & & & $\begin{array}{l}\text { Some young people are very satisfied with } \\
\text { themselves physically }\end{array}$ & BUT & $\begin{array}{l}\text { Other young people are often dissatisfied with } \\
\text { themselves physically. }\end{array}$ & & \\
\hline 9 & & & $\begin{array}{l}\text { Some young people don't like the way they } \\
\text { are leading their life }\end{array}$ & BUT & $\begin{array}{l}\text { Other young people do like the way they are } \\
\text { leading their life. }\end{array}$ & & \\
\hline 10 & & & $\begin{array}{l}\text { Some young people feel that they are often } \\
\text { admired for their good-looking bodies }\end{array}$ & BUT & $\begin{array}{l}\text { Other young people feel that they are seldom } \\
\text { admired for the way their bodies look. }\end{array}$ & & \\
\hline
\end{tabular}




\begin{tabular}{|c|c|c|c|c|c|c|c|}
\hline \multicolumn{2}{|r|}{$\begin{array}{l}\text { Really } \\
\text { true for } \\
\text { me }\end{array}$} & $\begin{array}{l}\text { Sort of } \\
\text { true for }\end{array}$ & \multicolumn{3}{|c|}{ Remember: only tick ONE box for each question please } & \multirow{2}{*}{$\begin{array}{c}\begin{array}{c}\text { Sort of } \\
\text { true for } \\
\text { me }\end{array} \\
\square\end{array}$} & \multirow{2}{*}{$\begin{array}{c}\text { Really } \\
\text { true for } \\
\text { me }\end{array}$} \\
\hline 11 & & & $\begin{array}{l}\text { Some young people are happy with how } \\
\text { they are and what they can do physically }\end{array}$ & BUT & $\begin{array}{l}\text { Other young people are unhappy with how } \\
\text { they are and what they can do physically. }\end{array}$ & & \\
\hline 12 & & & $\begin{array}{l}\text { Some young people like the kind of person } \\
\text { they are }\end{array}$ & BUT & $\begin{array}{l}\text { Other young people often wish they were } \\
\text { someone else. }\end{array}$ & & \\
\hline 13 & & & $\begin{array}{l}\text { Some young people are confident about } \\
\text { how their bodies look physically }\end{array}$ & BUT & $\begin{array}{l}\text { Other young people feel uneasy about how } \\
\text { their bodies look physically. }\end{array}$ & & \\
\hline 14 & & & $\begin{array}{l}\text { Some young people have a positive feeling } \\
\text { about themselves physically }\end{array}$ & BUT & $\begin{array}{l}\text { Other young people feel somewhat negative } \\
\text { about themselves physically. }\end{array}$ & & \\
\hline 15 & & & $\begin{array}{l}\text { Some young people are very happy being } \\
\text { the way they are }\end{array}$ & BUT & Other young people wish they were different & & \\
\hline 16 & & & $\begin{array}{l}\text { Some young people don't like how their } \\
\text { bodies look physically }\end{array}$ & BUT & $\begin{array}{l}\text { Other young people are pleased with how } \\
\text { their bodies look physically. }\end{array}$ & & \\
\hline 17 & & & $\begin{array}{l}\text { Some young people wish that they could } \\
\text { feel better about themselves physically }\end{array}$ & BUT & $\begin{array}{l}\text { Other young people always seem to feel good } \\
\text { about themselves physically. }\end{array}$ & & \\
\hline 18 & & & $\begin{array}{l}\text { Some young people are not very happy } \\
\text { with the way they do a lot of things }\end{array}$ & BUT & $\begin{array}{l}\text { Other young people think the way they do } \\
\text { things is fine. }\end{array}$ & & \\
\hline 19 & & & $\begin{array}{l}\text { Some young people think it's very important to } \\
\text { have a good-looking (fit-looking) body in order } \\
\text { to feel good about themselves as a person }\end{array}$ & BUT & $\begin{array}{l}\text { Other young people don't think that having a } \\
\text { good-looking body is important at all. }\end{array}$ & & \\
\hline 20 & & & $\begin{array}{l}\text { Some young people dont think that having } \\
\text { a body that looks in good physical shape is } \\
\text { important to how they feel about } \\
\text { themselves }\end{array}$ & BUT & $\begin{array}{l}\text { Other young people feel that it's very } \\
\text { important to have a body that looks in good } \\
\text { physical shape. }\end{array}$ & & \\
\hline
\end{tabular}




\section{Appendix 4: Anthropometry Recording Form}



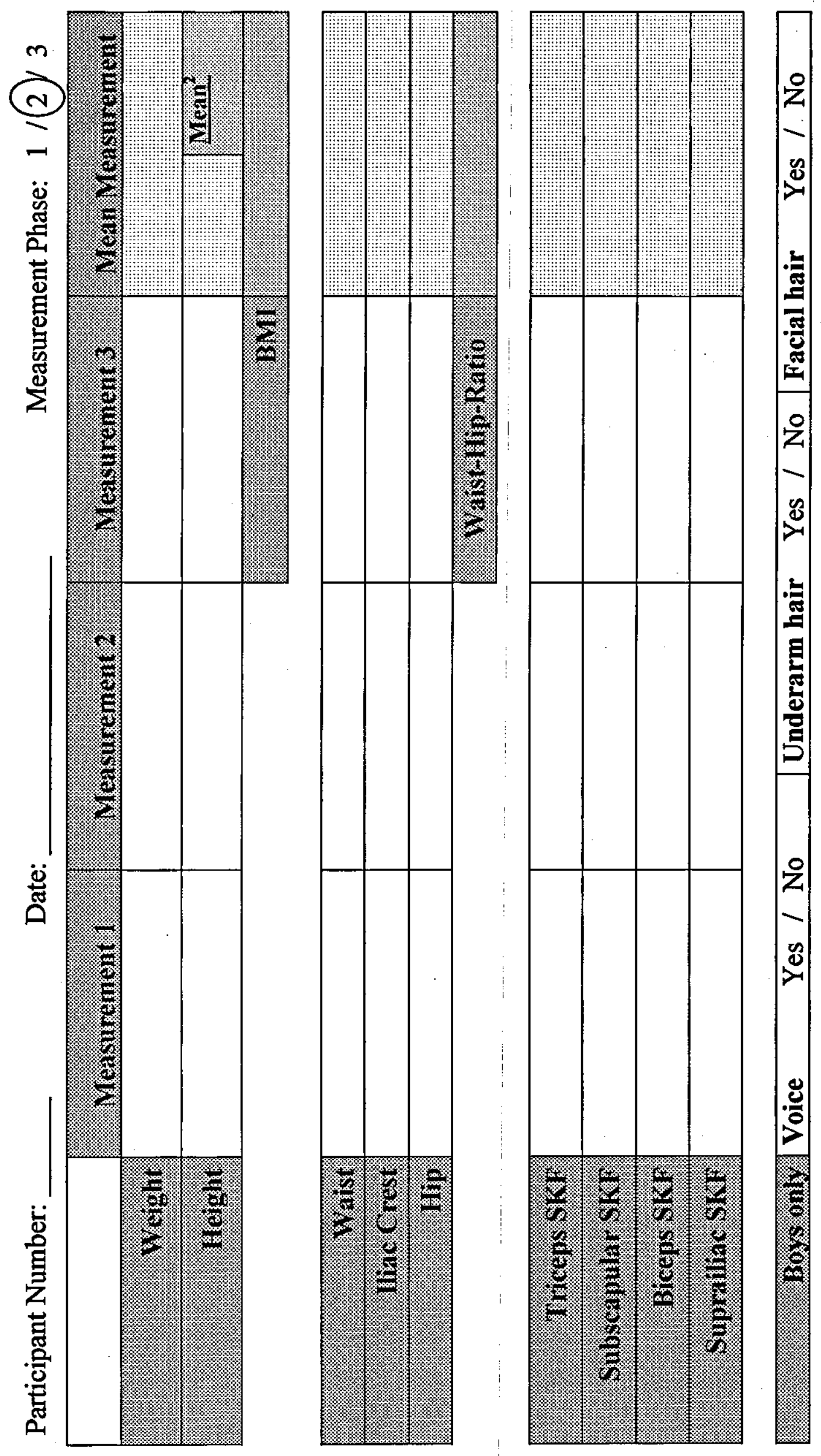

